NASA CR-178149

(SER-510237)

(NASA-CE-178149) FLIGHT SERVICE EVALUATION

N87-10182

OF COMPOSITE HEIICOPTER COHECNENTS AnRUal

Report, CCt. 1983 - DEC. 1985 (Sikorsky

Aircraft, Stratfcrd, Conn.) 66 p CSCL 11D

G3/24 44286

\title{
FLIGHT SERVICE \\ EVALUATION OF COMPOSITE \\ HELICOPTER COMPONENTS
}

Third Annual Report

OCTOBER 1983 through DECEMBER 1985

George H. Mardoian and Maureen B. Ezzo

SIKORSKY AIRCRAFT

DIVISION OF UNITED TECHNOLOGIES CORPORATION

Stratford, Connecticut 06602

CONTRACT NO. NASI-16542

SEPTEMBER 1986

N/Sก

Natıonal Aeronautics and

Space Admınistration

Langley Research Center

Hampton, Virginia 23665-5225 
NASA CR-178149

(SER-510237)

\section{FLIGHT SERVICE EVALUATION OF COMPOSITE HELICOPTER COMPONENTS \\ Third Annual Report \\ OCTOBER 1983 through DECEMBER 1985}

George H. Mardoian and Maureen B. Ezzo

September, 1986

Prepared under contract No. NASI-16542

by

SIKORSKY AIRCRAFT

DIVISION OF UNITED TECHNOLOGIES CORPORATION Stratford, Connecticut 06602

for

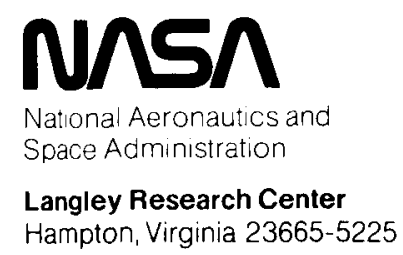




\section{FOREWORD}

This report was prepared by Sikorsky Aircraft, Division of United Technologies Corporation, Stratford, Connecticut 06602, under NASA Contract NAS1-16542 and covers the work performed during the period of October 1983 through December 1985. This program was jointly funded by the Materials Division of NASALangley Research Center and Aerostructures Directorate, U.S. Army Research and Technology Activity (AVSCOM). The contract is monitored by Mr. Donald J. Baker of the Applied Materials Branch.

The authors wish to acknowledge the contributions of the following Sikorsky personnel: W. G. Andrew and J. G. Byerley, component testing, E. Hansen component teardown, R. A. Pearsall, World Wide Customer Service and M. D. Rogers, graphical presentations. 
1.0

2.0

3.0

3.1

3.2

4.0

4.1

4.2

5.0

5.1

6.0

7.0
INTRODUCTION

IN-SERVICE COMPONENT SELECTION

TESTS OF IN-SERVICE COMPONENTS

Horizontal Stabilizers

Tail Rotor Spars

MATERIAL EVALUATION

Field Exposed Panels

Coupon Strength Tests

ANALYSIS OF TEST RESULTS

Environmental Factor Trends

CONCLUSIONS

REFERENCES
2

3

8

8

25

48

48

48

55

55

59

60 


\section{$\underline{\text { LIST OF TABLES }}$}

I Schedule for Evaluation of In-Service Environmental Effects on Advanced Composite Structures

II S-76 Components Available for Testing - Contract NASI -16542 B-157-00009, Buttlines 6-7

Moisture Desorption of Horizontal Stabilizer S/N B-157-00009, Buttlines 8-9

IX Spar S/N A-116-00178 Interlaminar Shear Static Coupon Testing at Room Temperature

X Spar S/N A-116-00178 Interlaminar Shear Static Coupon Testing at $170 \mathrm{~F}$

XI Compilation of Spar Coupon Static Testing with Data

XII Compilation of Spar Coupon Static Testing with Comparison to Panel Test Results - 170F Data 


\section{LIST OF FIGURES}

FIGURE

TITLE

PAGE

1

2

3

4

5

6

7

8

10

11

12

13

14

15

16

17
S-76 Tail Rotor Spar

4

5

11

18

Moisture Desorption of Stabilizer S/N B-157-00009

23

S-76 Stabilizer Location and Magnitude of Static Test Loads

Summary of S-76 Stabilizer Deflection and Moisture Data

24

S-76 Tail Rotor Spar Sketch of Coupon Locations 30

$\mathrm{T}$-Test Calculations to Determine if Test Results

32

From A and B Ends of Tail Rotor Spar S/N A-116-00178

Are From the Same Population

Spar S/N A-116-00178 Interlaminar Shear Fatigue

35

Coupon Testing - Maximum Stress Versus Cycles to

Fracture

Moisture Desorption of Tail Rotor Spar S/N

37

A-116-00178, Coupon 5-7-1, Right

Measured Moisture Versus Analysis for Graphite/

46

Epoxy Tail Rotor Spars

Correlation Between Measured Moisture and Analysis

47

For Graphite/Epoxy Tail Rotor Spars

Measured and Predicted Moisture Level for Six Ply

49

AS1/6350 Graphite Epoxy Panels (Weathered in

Stratford, Connecticut)

Life Extension Program Test Specimen Configurations

Comparison of Life Extension Program Flexural Shear

Environmental Factor Data with AS1/6350 Environmental

Factor Trends

Comparison of Life Extension Program Interlaminar

Shear (Static) Environmental Factor Data with AS1/6350 Environmental Factor Trends

Comparison of Life Extension Program Tension mental Factor Trends 


\author{
FLIGHT SERVICE EVALUATION OF COMPOSITE \\ HELICOPTER COMPONENTS \\ (Third Annual Report) \\ by \\ G.H. Mardoian and M.B. Ezzo \\ Sikorsky Aircraft \\ Division of United Technologies Corporation \\ Stratford, Connecticut 06602
}

\begin{abstract}
SUMMARY
This third annual report presents the environmental effects data for over four years service operation of an S-76 tail rotor spar, two horizontal stabilizers with four and a half and five and a half years of service operation and four and five year data for field exposed composite panels.

One S-76 tail rotor spar was returned with over four years (55 months) of commercial operation in the Louisiana Gulf Coast region. After the paddles were removed and the spar was nondestructively inspected it was cut up for small scale coupon testing. Both horizontal stabilizers passed the proof load and fatigue tests. The static small scale coupon test results compared favorably with those generated for the environmentally exposed panels.

Panels exposed for four years were returned for moisture measurements and strength tests. Environmental analyses were made of the returned tail rotor spars, horizontal stabilizers and composite panels. Both moisture and strength predictions were compared with measured data. The moisture absorption was close to predictions. The affect of outdoor exposure on strength was comparable to laboratory and conditioned strength data.
\end{abstract}


$1.0 \quad$ INTRODUCTION

This third annual flight service report is submitted in accordance with the requirements of contract NAS1-16542 and covers the progress made for the period of October 1983 through December 1985. A primary objective of this program is to evaluate the effect of moisture on the mechanical properties of composite materials subjected to long term environmental exposure.

This effort is part of an eight year program to evaluate the effects of environmental exposure on composite helicopter structures. As part of this effort, full scale tests are conducted on composite components removed from Sikorsky S-76 helicopters in commercial service in the Gulf Coast Region of Louisiana. Tail rotor spars and horizontal stabilizers are periodically returned from the operating environment for full scale static, fatigue, and small scale coupon testing. Full scale test results are compared to initial FAA certification data. The amount of moisture absorbed is determined and compared with expected values. Environmental factors calculated from small scale coupon tests are compared with data generated in testing coupons removed from composite panels, having the same ply configurations as the components, exposed to the outdoor environment in two weathering locations.

Work on this contract was initiated in 1981. This is the third report published to document the results of the program. The first annual report, Reference (1), covered the reporting period from March 1981 to April 1982. The second report, Reference (2), documented results from May 1982 to September 1983.

During this reporting period, a horizontal stabilizer having 56 months and 3999 flight hours was returned for fatigue testing. A second horizontal stabilizer having 66 months and 4213 flight hours was returned for static testing. A tail rotor spar with 55 months and 3752 flight hours was returned for small scale testing. Panels having four and five years of environmental exposure were returned from their locations for coupon testing.

Full details of the testing are documented in the sections to follow. 
The components selected for in-service evaluation were the tail rotor spar and the horizontal stabilizer. The tail rotor spar is constructed of uni-directional graphite/epoxy (Hercules ASl/Ciba Geigy 6350 ), ranging in thickness from 14 to 33 plies. The geometry is illustrated in Figure 1. The horizontal stabilizer is constructed of $\pm 45^{\circ}$ Kevlar/epoxy (285/American Cyanamid 5143) over Nomex honeycomb core with a torque box section fabricated of $\pm 45^{\circ}$ Kevlar/epoxy, aluminum honeycomb core and graphite/epoxy cap strip reinforcements. A schematic diagram of the stabilizer is shown in Figure 2.

Tail rotor spars and stabilizers are returned from the field for full scale static, full scale fatigue or small scale testing in accordance with the schedule detailed in Table $I$. A total of ten tail rotor spars and four stabilizers will be returned from the field for evaluation as required for this program.

The tail rotor spars and stabilizers to be evaluated are removed from S-76 aircraft owned and operated by Air Logistics in the Gulf Coast region of Louisiana. Every three months, the Air Logistics' aircraft logs are inspected to verify that each part being tracked is still installed on an operating aircraft. In addition to the components being tracked for testing, extra spars and stabilizers are tracked as spares, for use in the event that one of the components scheduled for testing becomes unavailable. The number of flight hours and months of in-service environmental exposure are recorded for each part and spare. An updated list of the tail rotor spars and horizontal stabilizers being tracked is presented in Table II.

During this reporting period, inspection of the aircraft logs revealed a tail rotor spar being tracked as a spare was no longer available for testing. The aircraft that tail rotor spar S/N A-11600438 was installed on was sold by Air Logistics. Owing to the fact that two other tail rotor spars are available as spares, the spar will not be replaced. 


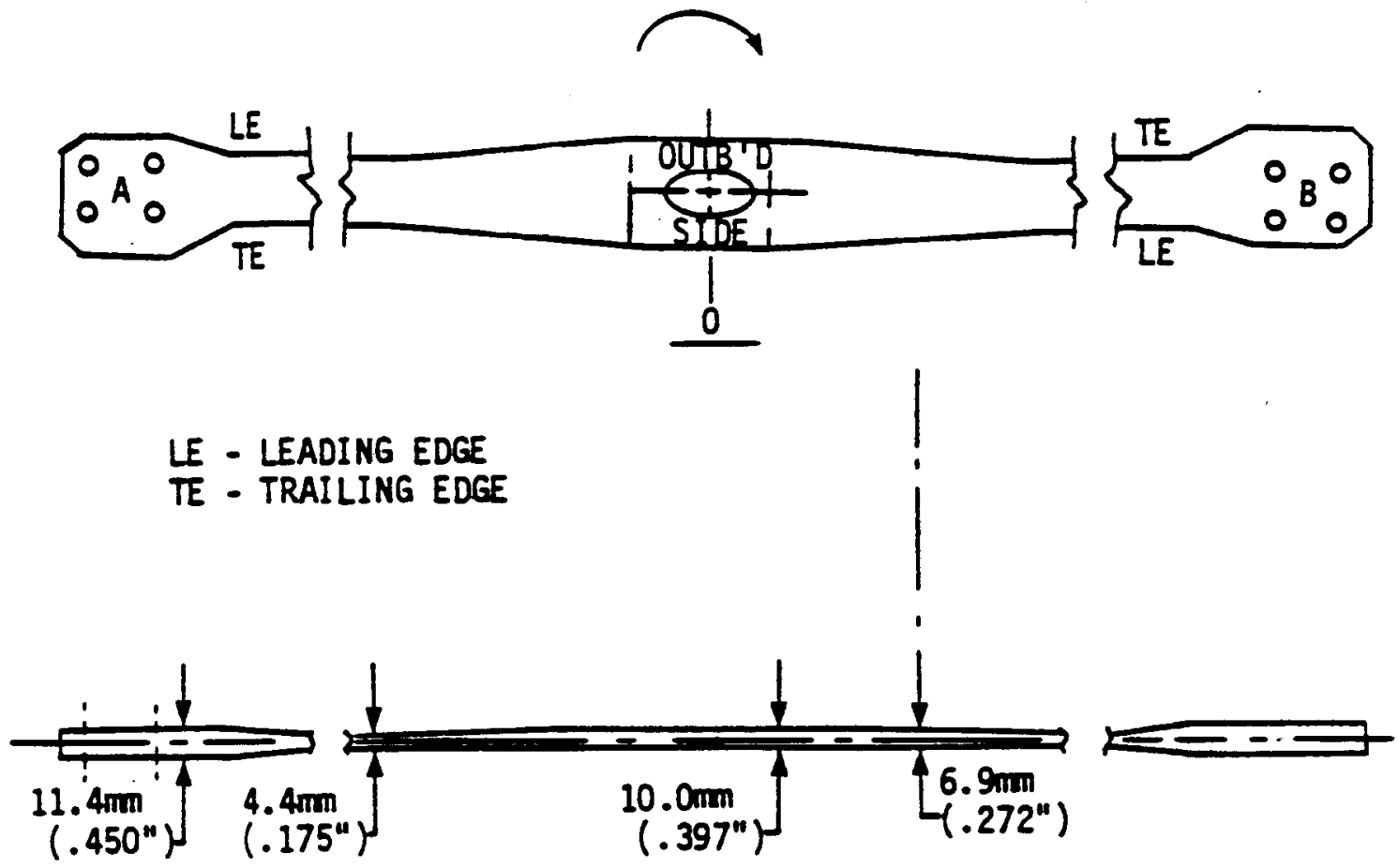

FIGURE 1. S-76 TAIL ROTOR SPAR 

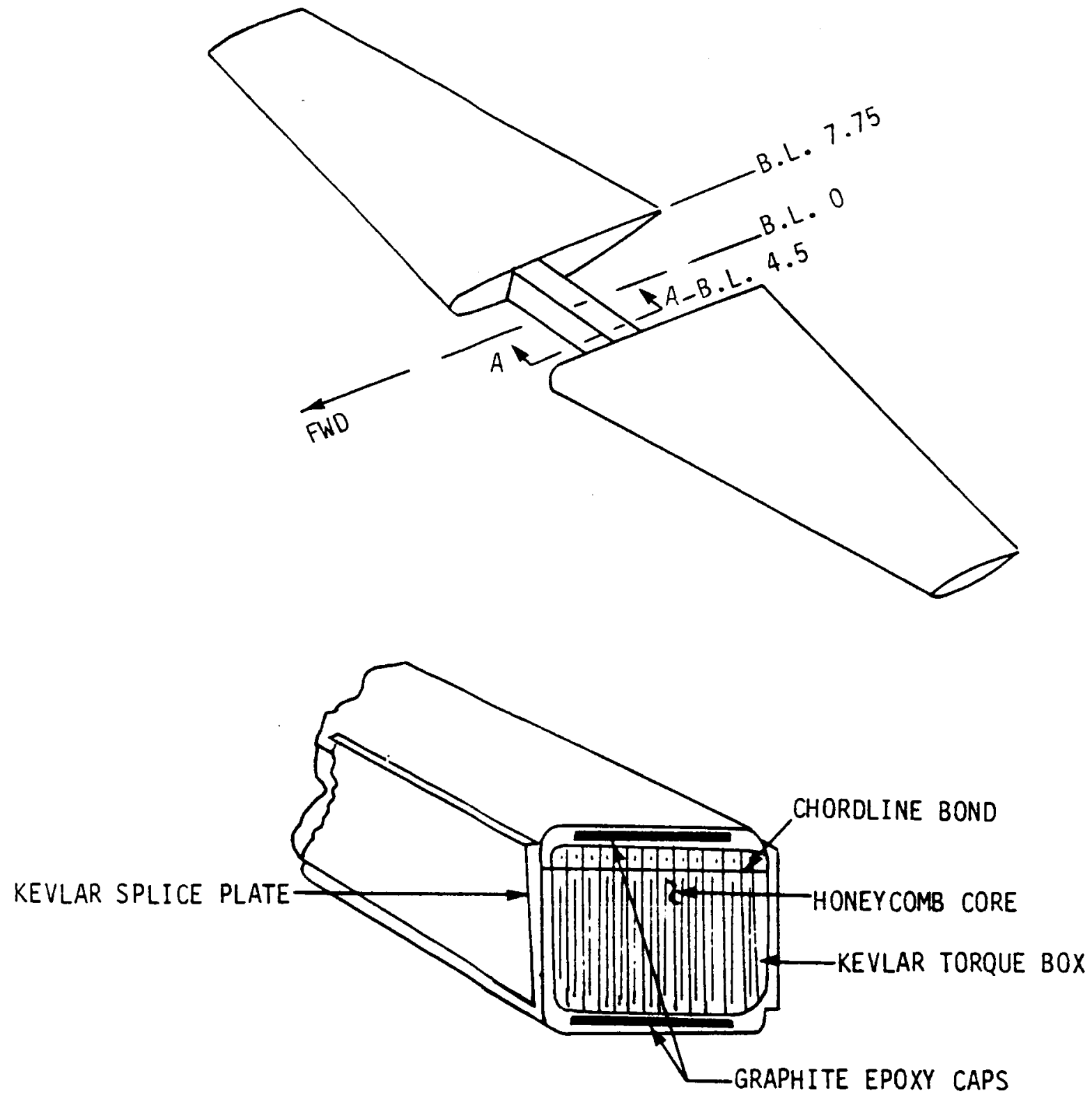

STABILIZER TORQUE BOX AT SECTION A-A

FIGURE 2. SCHEMATIC REPRESENTATION OF STABILIZER 
TABLE I SCHEDULE FOR EVALUATION OF IN-SERVICE ENVIRONMENTAL EFFECTS ON ADVANCED COMPOSITE STRUCTURES

S-76 HELICOPTER

NASA CONTRACT NAS1-16542

CALENDAR YEAR

$\begin{array}{llllllll}81 & 82 & 83 & 84 & 85 & 86 & 87 & 88\end{array}$

In-Service Component Selection

Tracking

$\begin{array}{llllllll}\mathrm{X} & \mathrm{X} & \mathrm{X} & \mathrm{X} & \mathrm{X} & \mathrm{X} & \mathrm{X} & \mathrm{X}\end{array}$

Selection:

Horizontal Stabilizer

Tail Rotor Spar

$\begin{array}{llllllll}X & & X & & X & & X & \\ X & X & X & X & X & X & X & X\end{array}$

Tests of In-Service Components

Horizontal Stabilizers:

Fatigue Tests, Full Scale

Static Tests, Full Scale

$\begin{array}{llll} & X & & X \\ X & X & X & X\end{array}$

Tail Rotor Spars:

Fatigue Tests, Full Scale

Coupon Tests, Small Scale

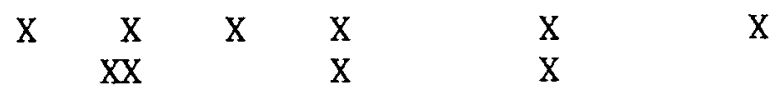

Material Evaluation

$\begin{array}{lllllllll}X & X & X & X & X & X & X & X\end{array}$

Analysis of Test Results

$\begin{array}{llllllllll}X & X & X & X & X & X & X & X\end{array}$ 
Table II. S-76 Components Available for Testing - Contract NAS1-16542

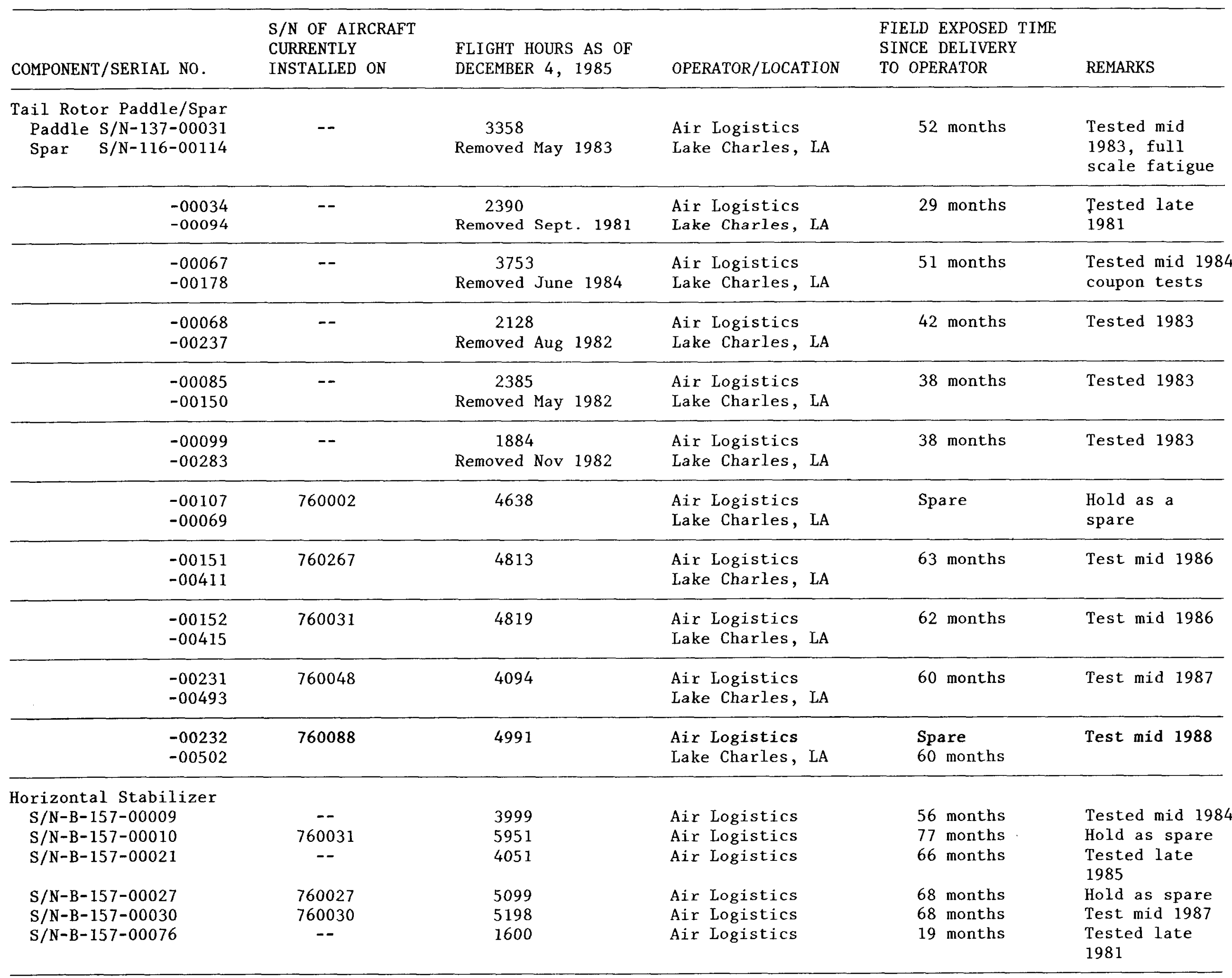

要 


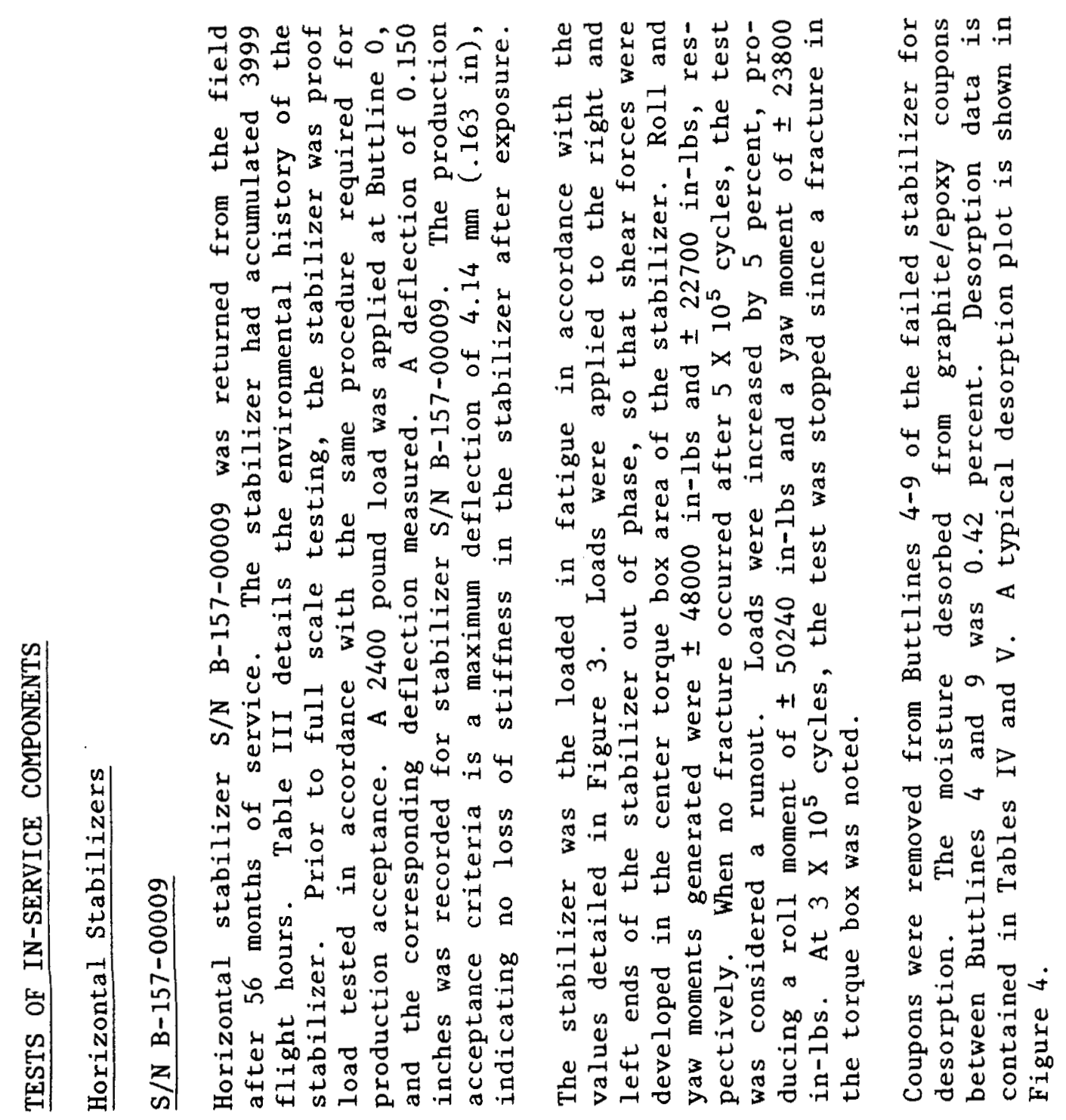

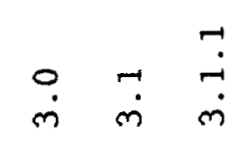


TABLE III

STABILIZER S/N B-157-00009

SUMMARY OF ENVIRONMENTAL HISTORY

\begin{tabular}{|c|c|c|c|c|}
\hline \multicolumn{2}{|r|}{ DATE } & \multicolumn{2}{|c|}{$\begin{array}{c}\text { AVERAGE } \\
\text { TEMPERATURE }\end{array}$} & $\begin{array}{c}\text { AVERAGE } \\
\text { RELATIVE HUMIDITY } \\
(\%)\end{array}$ \\
\hline $1 / 10 / 79$ & $-\quad 1 / 31 / 79$ & 6.4 & 43.6 & 72.6 \\
\hline $2 / 01 / 79$ & $-\quad 2 / 28 / 79$ & 9.8 & 49.7 & 82.1 \\
\hline $3 / 01 / 79$ & $-3 / 31 / 79$ & 15.9 & 60.7 & 74.5 \\
\hline $4 / 01 / 79$ & $-\quad 4 / 30 / 79$ & 20.1 & 68.1 & 80.5 \\
\hline $5 / 01 / 79$ & $-5 / 31 / 79$ & 22.4 & 72.3 & 78.6 \\
\hline $6 / 01 / 79$ & $-6 / 30 / 79$ & 26.1 & 78.9 & 78.4 \\
\hline $7 / 01 / 79$ & $-7 / 31 / 79$ & 26.8 & 80.3 & 85.4 \\
\hline $8 / 01 / 79$ & $-\quad 8 / 31 / 79$ & 26.7 & 80.0 & 83.8 \\
\hline $9 / 01 / 79$ & $-\quad 9 / 30 / 79$ & 23.7 & 74.7 & 80.3 \\
\hline $10 / 01 / 79$ & $-10 / 31 / 79$ & 20.5 & 68.9 & 79.0 \\
\hline $11 / 01 / 79$ & $-11 / 30 / 79$ & 12.4 & 54.4 & 75.4 \\
\hline $12 / 01 / 79$ & $-12 / 31 / 79$ & 10.3 & 50.5 & 78.1 \\
\hline $1 / 01 / 80$ & $-\quad 1 / 31 / 80$ & 11.9 & 53.4 & 86.4 \\
\hline $2 / 01 / 80$ & $-2 / 29 / 80$ & 10.3 & 50.6 & 80.5 \\
\hline $3 / 01 / 80$ & $-\quad 3 / 31 / 80$ & 15.2 & 59.4 & 81.4 \\
\hline $4 / 01 / 80$ & $-4 / 30 / 80$ & 18.4 & 65.1 & 76.5 \\
\hline $5 / 01 / 80$ & $-5 / 31 / 80$ & 23.8 & 74.8 & 83.9 \\
\hline $6 / 01 / 80$ & $-6 / 30 / 80$ & 27.1 & 80.8 & 80.3 \\
\hline $7 / 01 / 80$ & $-7 / 31 / 80$ & 28.2 & 82.8 & 72.5 \\
\hline $8 / 01 / 80$ & $-8 / 31 / 80$ & 27.4 & 81.3 & 74.0 \\
\hline $9 / 01 / 80$ & $-\quad 9 / 30 / 80$ & 26.3 & 79.4 & 79.3 \\
\hline $10 / 01 / 80$ & $-10 / 31 / 80$ & 18.0 & 64.4 & 69.8 \\
\hline $11 / 01 / 80$ & $-11 / 30 / 80$ & 12.7 & 54.8 & 78.0 \\
\hline $12 / 01 / 80$ & $-12 / 31 / 80$ & 10.7 & 51.3 & 75.0 \\
\hline $1 / 01 / 81$ & $-1 / 31 / 81$ & 8.2 & 46.8 & 73.5 \\
\hline $2 / 01 / 81$ & $-\quad 2 / 28 / 81$ & 11.1 & 52.0 & 74.0 \\
\hline $3 / 01 / 81$ & $-3 / 31 / 81$ & 14.9 & 58.9 & 66.4 \\
\hline $4 / 01 / 81$ & $-4 / 30 / 81$ & 21.4 & 70.5 & 76.1 \\
\hline $5 / 01 / 81$ & $-\quad 5 / 31 / 81$ & 22.6 & 72.6 & 73.3 \\
\hline $6 / 01 / 81$ & $-\quad 6 / 30 / 81$ & 26.8 & 80.3 & 82.1 \\
\hline $7 / 01 / 81$ & $-7 / 31 / 81$ & 27.3 & 81.1 & 81.8 \\
\hline $8 / 01 / 81$ & $-\quad 8 / 31 / 81$ & 26.9 & 80.5 & 79.3 \\
\hline $9 / 01 / 81$ & $-\quad 9 / 30 / 81$ & 23.8 & 74.8 & 77.3 \\
\hline $10 / 01 / 81$ & $-10 / 31 / 81$ & 20.1 & 68.1 & 79.1 \\
\hline $11 / 01 / 81$ & $-11 / 30 / 81$ & 16.1 & 60.9 & 80.9 \\
\hline $12 / 01 / 81$ & $-12 / 31 / 81$ & 11.4 & 52.5 & 73.4 \\
\hline
\end{tabular}


TABLE III (Continued)

STABILIZER S/N B-157-00009

SUMMARY OF ENVIRONMENTAL HISTORY

\begin{tabular}{|c|c|c|c|c|}
\hline \multicolumn{2}{|c|}{ DATE } & \multicolumn{2}{|c|}{$\begin{array}{c}\text { AVERAGE } \\
\text { TEMPERATURE }\end{array}$} & \multirow{2}{*}{$\begin{array}{l}\text { AVERAGE } \\
\text { RELATIVE HUMIDITY } \\
(\%) \\
76.9\end{array}$} \\
\hline $1 / 01 / 82$ & $-\quad 1 / 31 / 82^{\circ}$ & 11.1 & 51.9 & \\
\hline $2 / 01 / 82$ & $-\quad 2 / 28 / 82$ & 10.8 & 51.4 & 78.4 \\
\hline $3 / 01 / 82$ & $-\quad 3 / 31 / 82$ & 16.9 & 62.5 & 82.6 \\
\hline $4 / 01 / 82$ & $-\quad 4 / 30 / 82$ & 18.9 & 66.1 & 80.1 \\
\hline $5 / 01 / 82$ & - $5 / 31 / 82$ & 23.2 & 73.8 & 82.1 \\
\hline $6 / 01 / 82$ & $-6 / 30 / 82$ & 26.4 & 79.6 & 82.4 \\
\hline $7 / 01 / 82$ & $-7 / 31 / 82$ & 27.2 & 80.9 & 80.8 \\
\hline $8 / 01 / 82$ & $-\quad 8 / 31 / 82$ & 26.9 & 80.5 & 78.8 \\
\hline $9 / 01 / 82$ & $-\quad 9 / 30 / 82$ & 24.2 & 75.6 & 75.5 \\
\hline $10 / 01 / 82$ & $-10 / 31 / 82$ & 20.2 & 68.3 & 70.9 \\
\hline $11 / 01 / 82$ & $-11 / 30 / 82$ & 16.4 & 61.5 & 74.3 \\
\hline $12 / 01 / 82$ & $-12 / 31 / 82$ & 13.9 & 57.0 & 81.1 \\
\hline $1 / 01 / 83$ & $-\quad 1 / 31 / 83$ & 9.5 & 49.1 & 81.1 \\
\hline $2 / 01 / 83$ & $-\quad 2 / 28 / 83$ & 11.3 & 52.4 & 77.3 \\
\hline $3 / 01 / 83$ & $-\quad 3 / 31 / 83$ & 14.2 & 57.6 & 73.5 \\
\hline $4 / 01 / 83$ & $-4 / 30 / 83$ & 17.5 & 63.5 & 73.4 \\
\hline $5 / 01 / 83$ & $-\quad 5 / 31 / 83$ & 23.0 & 73.4 & 77.1 \\
\hline $6 / 01 / 83$ & $-6 / 30 / 83$ & 25.6 & 78.0 & 81.3 \\
\hline $7 / 01 / 83$ & $-7 / 31 / 83$ & 28.2 & 82.8 & 78.1 \\
\hline $8 / 01 / 83$ & $-\quad 8 / 31 / 83$ & 27.8 & 82.1 & 81.4 \\
\hline
\end{tabular}




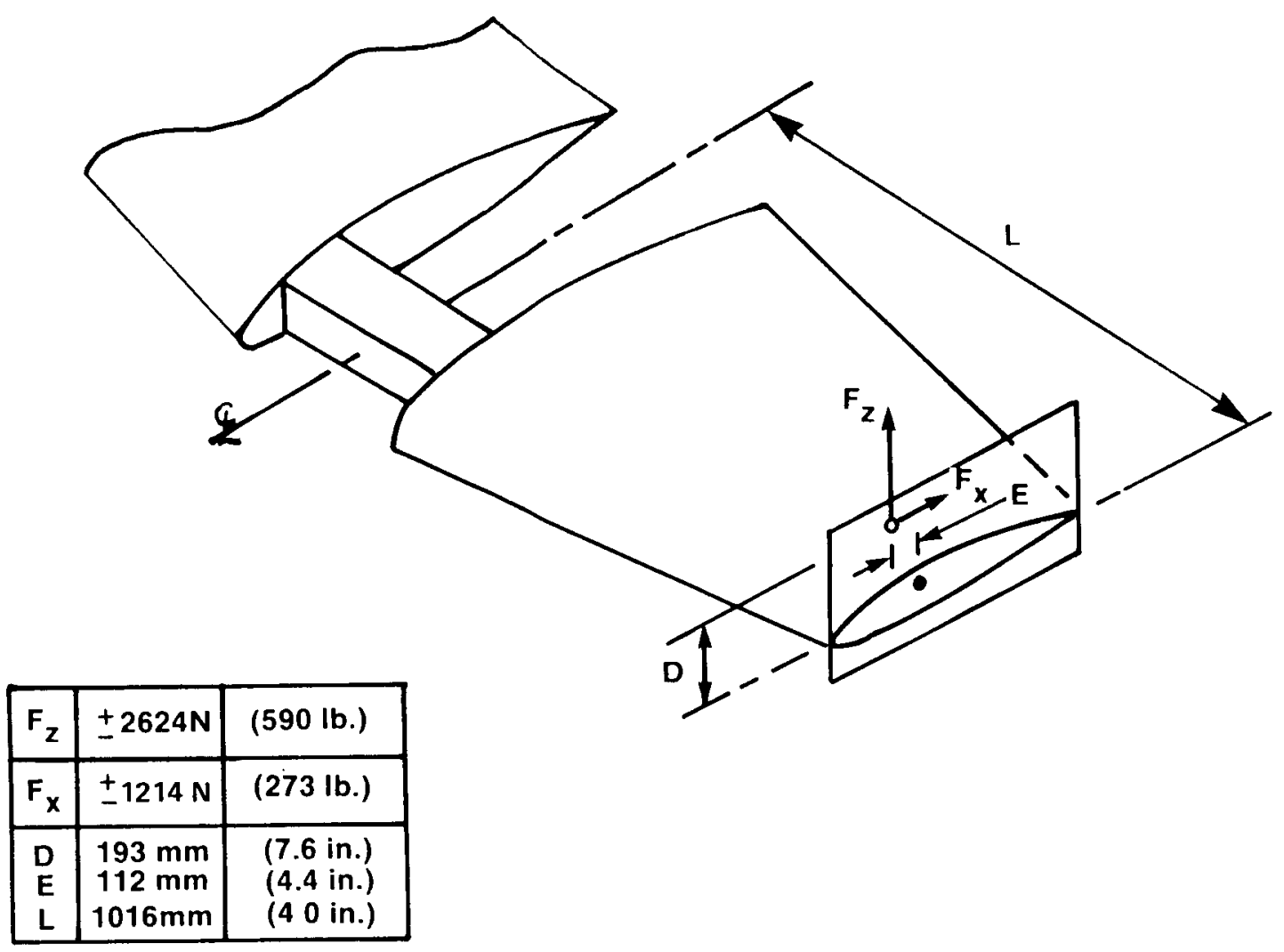

FIGURE 3

S-76 STABILIZER LOCATION AND

MAGNITUDE OF FATIGUE TEST LOADS 
TABLE IV MOISTURE DESORPTION OF HORIZONTAL STABILIZER S/N B-157-00009, BUTTLINES 6-7

\begin{tabular}{|c|c|c|c|c|c|c|c|}
\hline $\begin{array}{l}\text { DATE } \\
\text { OF } \\
\text { WEIGHING }\end{array}$ & DAYS & $\begin{array}{c}\text { SQRT } \\
\text { OF } \\
\text { TIME }\end{array}$ & $\begin{array}{l}\% \text { MOIST } \\
\text { DESORBED } \\
\text { COUP 67B }\end{array}$ & $\begin{array}{l}\% \text { MOIST } \\
\text { DESORBED } \\
\text { COUP } 67 T\end{array}$ & $\begin{array}{l}\text { WE IGHT } \\
\text { OF } \\
\text { COUP } 67 \mathrm{~B}\end{array}$ & $\begin{array}{l}\text { WEIGHT } \\
\text { OF } \\
\text { COUP } 67 T\end{array}$ & $\begin{array}{l}\text { AVERAGE } \\
\% \text { MOIST } \\
\text { DESORBED }\end{array}$ \\
\hline $3 / 12 / 84$ & 0 & 0 & 0 & 0 & 10.2752 & 9.9427 & 0 \\
\hline $3 / 14 / 84$ & 2 & 1.41 & -0.11 & -0.09 & 10.2644 & 9.9335 & -0.10 \\
\hline $3 / 16 / 84$ & 4 & 2.00 & -0.16 & -0.11 & 10.2592 & 9.9315 & -0.13 \\
\hline $3 / 19 / 84$ & 7 & 2.65 & -0.17 & -0.15 & 10.2573 & 9.9277 & -0.16 \\
\hline $3 / 21 / 84$ & 9 & 3.00 & -0.17 & -0.15 & 10.258 & 9.9278 & -0.16 \\
\hline $3 / 23 / 84$ & 11 & 3.32 & -0.19 & -0.19 & 10.2557 & 9.9241 & -0.19 \\
\hline $3 / 28 / 84$ & 16 & 4.00 & -0.25 & -0.22 & 10.2496 & 9.9213 & -0.23 \\
\hline $3 / 30 / 84$ & 18 & 4.24 & -0.23 & -0.19 & 10.2515 & 9.9234 & -0.21 \\
\hline $4 / 2 / 84$ & 21 & 4.58 & -0.24 & -0.20 & 10.2503 & 9.9225 & -0.22 \\
\hline $4 / 4 / 84$ & 23 & 4.80 & -0.26 & -0.22 & 10.2482 & 9.9206 & -0.24 \\
\hline $4 / 6 / 84$ & 25 & 5.00 & -0.27 & -0.22 & 10.2479 & 9.9206 & -0.24 \\
\hline $4 / 9 / 84$ & 28 & 5.29 & -0.27 & -0.23 & 10.2478 & 9.9195 & -0.25 \\
\hline $4 / 11 / 84$ & 30 & 5.48 & -0.30 & -0.26 & 10.2443 & 9.9166 & -0.28 \\
\hline $4 / 13 / 84$ & 32 & 5.66 & -0.28 & -0.24 & 10.2464 & 9.9193 & -0.26 \\
\hline $4 / 16 / 84$ & 35 & 5.92 & -0.29 & -0.24 & 10.2458 & 9.9192 & -0.26 \\
\hline $4 / 23 / 84$ & 42 & 6.48 & -0.31 & -0.27 & 10.243 & 9.9163 & -0.29 \\
\hline $4 / 27 / 84$ & 46 & 6.78 & -0.31 & -0.27 & 10.2436 & 9.916 & -0.29 \\
\hline $4 / 30 / 84$ & 49 & 7.00 & -0.32 & -0.27 & 10.2422 & 9.9158 & -0.30 \\
\hline $5 / 4 / 84$ & 53 & 7.28 & -0.32 & -0.27 & 10.2428 & 9.9156 & -0.29 \\
\hline $5 / 7 / 84$ & 56 & 7.48 & -0.31 & -0.27 & 10.2433 & 9.9154 & -0.29 \\
\hline $5 / 11 / 84$ & 60 & 7.75 & -0.34 & -0.30 & 10.2403 & 9.9129 & -0.32 \\
\hline $5 / 14 / 84$ & 63 & 7.94 & -0.34 & -0.30 & 10.2402 & 9.9126 & -0.32 \\
\hline $5 / 21 / 84$ & 70 & 8.27 & -0.34 & -0.30 & 10.2398 & 9.913 & -0.32 \\
\hline $5 / 25 / 84$ & 74 & 8.60 & -0.34 & -0.28 & 10.2403 & 9.9145 & -0.31 \\
\hline $6 / 18 / 84$ & 98 & 9.90 & -0.32 & -0.28 & 10.2419 & 9.9153 & -0.30 \\
\hline $6 / 22 / 84$ & 102 & 10.10 & -0.33 & -0.28 & 10.2409 & 9.9147 & -0.31 \\
\hline $6 / 26 / 84$ & 106 & 10.30 & -0.33 & -0.28 & 10.2418 & 9.915 & -0.30 \\
\hline $6 / 28 / 84$ & 108 & 10.39 & -0.36 & -0.31 & 10.2382 & 9.9121 & -0.33 \\
\hline $7 / 2 / 84$ & 112 & 10.58 & -0.37 & -0.32 & 10.2374 & 9.9107 & -0.34 \\
\hline $7 / 9 / 84$ & 119 & 10.91 & -0.36 & -0.31 & 10.2381 & 9.9118 & -0.34 \\
\hline $7 / 13 / 84$ & 123 & 11.09 & -0.36 & -0.31 & 10.238 & 9.9116 & -0.34 \\
\hline $7 / 16 / 84$ & 126 & 11.22 & -0.36 & -0.31 & 10.2381 & 9.9116 & -0.34 \\
\hline $7 / 18 / 84$ & 128 & 11.31 & -0.36 & -0.31 & 10.2384 & 9.9114 & -0.34 \\
\hline $7 / 20 / 84$ & 130 & 11.40 & -0.37 & -0.33 & 10.2372 & 9.9102 & -0.35 \\
\hline $7 / 23 / 84$ & 133 & 11.53 & -0.35 & -0.31 & 10.2392 & 9.9121 & -0.33 \\
\hline $7 / 27 / 84$ & 137 & 11.70 & -0.36 & -0.31 & 10.2384 & 9.9119 & -0.33 \\
\hline $7 / 30 / 84$ & 140 & 11.83 & -0.33 & -0.28 & 10.2413 & 9.9148 & -0.31 \\
\hline $8 / 3 / 84$ & 144 & 12.00 & -0.35 & -0.31 & 10.2388 & 9.9114 & -0.33 \\
\hline $8 / 6 / 84$ & 147 & 12.12 & -0.35 & -0.31 & 10.2392 & 9.9118 & -0.33 \\
\hline $8 / 10 / 84$ & 151 & 12.29 & -0.36 & -0.32 & 10.2386 & 9.9111 & -0.34 \\
\hline $8 / 13 / 84$ & 154 & 12.41 & -0.33 & -0.29 & 10.2408 & 9.9138 & -0.31 \\
\hline $8 / 17 / 84$ & 158 & 12.57 & -0.36 & -0.32 & 10.2382 & 9.9112 & -0.34 \\
\hline $8 / 20 / 84$ & 161 & 12.69 & -0.35 & -0.30 & 10.2388 & 9.9127 & -0.33 \\
\hline
\end{tabular}


TABLE IV (cont'd) MOISTURE DESORPTION OF HORIZONTAL STABILIZER S/N B-157-00009, BUTTLINES 6-7

\begin{tabular}{|c|c|c|c|c|c|c|c|}
\hline $\begin{array}{c}\text { DATE } \\
\text { OF } \\
\text { WEIGHING }\end{array}$ & DAYS & $\begin{array}{l}\text { SQRT } \\
\text { OF } \\
\text { TIME }\end{array}$ & $\begin{array}{l}\% \text { MOIST } \\
\text { DESORBED } \\
\text { COUP 67B }\end{array}$ & $\begin{array}{l}\% \text { MOIST } \\
\text { DESORBED } \\
\text { COUP 67T }\end{array}$ & $\begin{array}{l}\text { WEIGHT } \\
\text { OF } \\
\text { COUP } 67 \mathrm{~B}\end{array}$ & $\begin{array}{l}\text { WE IGHT } \\
\text { OF } \\
\text { COUP } 67 T\end{array}$ & $\begin{array}{l}\text { AVERAGE } \\
\% \text { MOIST } \\
\text { DESORBED }\end{array}$ \\
\hline $8 / 24 / 84$ & 165 & 12.85 & -0.37 & -0.33 & 10.2376 & 9.9103 & -0.35 \\
\hline $8 / 27 / 84$ & 168 & 12.96 & -0.36 & -0.30 & 10.2387 & 9.9128 & -0.33 \\
\hline $8 / 31 / 84$ & 172 & 13.11 & -0.34 & -0.30 & 10.2407 & 9.9127 & -0.32 \\
\hline $9 / 7 / 84$ & 179 & 13.38 & -0.37 & -0.32 & 10.2374 & 9.9113 & -0.34 \\
\hline $9 / 10 / 84$ & 182 & 13.49 & -0.36 & -0.31 & 10.2381 & 9.9114 & -0.34 \\
\hline $9 / 14 / 84$ & 186 & 13.64 & -0.35 & -0.30 & 10.2396 & 9.9133 & -0.32 \\
\hline $9 / 17 / 84$ & 189 & 13.75 & -0.38 & -0.32 & 10.2361 & 9.9109 & -0.35 \\
\hline $9 / 21 / 84$ & 193 & 13.89 & -0.38 & -0.32 & 10.2359 & 9.9107 & -0.35 \\
\hline $9 / 24 / 84$ & 196 & 14.00 & -0.36 & -0.30 & 10.2384 & 9.9131 & -0.33 \\
\hline $9 / 28 / 84$ & 200 & 14.14 & -0.37 & -0.33 & 10.2373 & 9.9102 & -0.35 \\
\hline $10 / 1 / 84$ & 203 & 14.25 & -0.38 & -0.32 & 10.2363 & 9.9106 & -0.35 \\
\hline $10 / 5 / 84$ & 207 & 14.39 & -0.38 & -0.32 & 10.2366 & 9.9111 & -0.35 \\
\hline $10 / 8 / 84$ & 210 & 14.49 & -0.39 & -0.33 & 10.2355 & 9.9096 & -0.36 \\
\hline $10 / 12 / 84$ & 214 & 14.63 & -0.40 & -0.34 & 10.2346 & 9.9085 & -0.37 \\
\hline $10 / 15 / 84$ & 217 & 14.73 & -0.30 & -0.34 & 10.2343 & 9.9084 & -0.37 \\
\hline $10 / 19 / 84$ & 221 & 14.87 & -0.39 & -0.34 & 10.2348 & 9.9091 & -0.37 \\
\hline $10 / 22 / 84$ & 224 & 14.97 & -0.38 & -0.33 & 10.2362 & 9.9095 & -0.36 \\
\hline $10 / 26 / 84$ & 228 & 15.10 & -0.37 & -0.32 & 10.2372 & 9.9106 & -0.35 \\
\hline $10 / 29 / 84$ & 231 & 15.20 & -0.37 & -0.32 & 10.2375 & 9.9106 & -0.34 \\
\hline $11 / 5 / 84$ & 238 & 15.43 & -0.37 & -0.31 & 10.2374 & 9.9119 & -0.34 \\
\hline $11 / 9 / 84$ & 242 & 15.56 & -0.37 & -0.32 & 10.237 & 9.9106 & -0.35 \\
\hline $11 / 12 / 84$ & 245 & 15.65 & -0.36 & -0.31 & 10.2381 & 9.9119 & -0.34 \\
\hline $11 / 16 / 84$ & 249 & 15.78 & -0.37 & -0.31 & 10.2371 & 9.9114 & -0.34 \\
\hline $11 / 19 / 84$ & 252 & 15.87 & -0.39 & -0.33 & 10.2351 & 9.9096 & -0.36 \\
\hline $11 / 26 / 84$ & 259 & 16.09 & -0.39 & -0.33 & 10.2354 & 9.9098 & -0.36 \\
\hline $11 / 30 / 84$ & 263 & 16.22 & -0.39 & -0.33 & 10.2352 & 9.9095 & -0.36 \\
\hline $12 / 7 / 84$ & 270 & 16.43 & -0.41 & -0.37 & 10.2326 & 9.9062 & -0.39 \\
\hline $12 / 10 / 84$ & 273 & 16.52 & 0.41 & -0.36 & 10.2335 & 9.9074 & -0.38 \\
\hline $12 / 14 / 84$ & 277 & 16.64 & -0.41 & -0.34 & 10.2335 & 9.9084 & -0.38 \\
\hline $12 / 17 / 84$ & 280 & 16.73 & -0.40 & -0.34 & 10.2337 & 9.9086 & -0.37 \\
\hline $1 / 4 / 85$ & 298 & 17.26 & -0.42 & -0.36 & 10.232 & 9.9065 & -0.39 \\
\hline $1 / 7 / 85$ & 301 & 17.35 & -0.43 & -0.38 & 10.2306 & 9.9053 & -0.41 \\
\hline $1 / 11 / 85$ & 305 & 17.46 & -0.43 & -0.37 & 10.231 & 9.9057 & -0.40 \\
\hline $1 / 18 / 85$ & 312 & 17.66 & -0.43 & -0.37 & 10.2307 & 9.9056 & -0.40 \\
\hline $1 / 21 / 85$ & 315 & 17.75 & -0.44 & -0.39 & 10.2303 & 9.9036 & -0.42 \\
\hline $1 / 25 / 85$ & 319 & 17.86 & -0.44 & -0.38 & 10.2295 & 9.9049 & -0.41 \\
\hline $1 / 28 / 85$ & 322 & 17.94 & -0.44 & -0.39 & 10.2299 & 9.9044 & -0.41 \\
\hline $2 / 1 / 85$ & 326 & 18.06 & -0.42 & -0.37 & 10.2318 & 9.9056 & -0.40 \\
\hline $2 / 4 / 85$ & 329 & 18.14 & -0.44 & -0.39 & 10.2296 & 9.9044 & -0.41 \\
\hline $2 / 8 / 85$ & 333 & 18.25 & -0.44 & -0.39 & $10.2299^{\circ}$ & 9.9041 & -0.41 \\
\hline $2 / 11 / 85$ & 336 & 18.33 & -0.46 & -0.40 & 10.228 & 9.9033 & -0.43 \\
\hline $2 / 15 / 85$ & 340 & 18.44 & -0.46 & -0.40 & 10.2279 & 9.9029 & -0.43 \\
\hline
\end{tabular}


TABLE IV (cont'd) MOISTURE DESORPTION OF HORIZONTAL STABILIZER S/N B-157-00009, BUTTLINES 6-7

\begin{tabular}{|c|c|c|c|c|c|c|c|}
\hline $\begin{array}{c}\text { DATE } \\
\text { OF } \\
\text { WE IGHING }\end{array}$ & DAYS & $\begin{array}{l}\text { SQRT } \\
\text { OF } \\
\text { TIME }\end{array}$ & $\begin{array}{l}\% \text { MOIST } \\
\text { DESORBED } \\
\text { COUP 67B }\end{array}$ & $\begin{array}{l}\% \text { MOIST } \\
\text { DESORBED } \\
\text { COUP } 67 T\end{array}$ & $\begin{array}{l}\text { WEIGHT } \\
\text { OF } \\
\text { COUP } 67 \mathrm{~B}\end{array}$ & $\begin{array}{l}\text { WEIGHT } \\
\text { OF } \\
\text { COUP } 67 \mathrm{~T}\end{array}$ & $\begin{array}{l}\text { AVERAGE } \\
\% \text { MOIST } \\
\text { DESORBED }\end{array}$ \\
\hline $2 / 18 / 85$ & 343 & 18.52 & -0.47 & -0.41 & 10.2271 & 9.9018 & -0.44 \\
\hline $2 / 22 / 85$ & 347 & 18.63 & -0.46 & -0.40 & 10.2279 & 9.903 & -0.43 \\
\hline $2 / 25 / 85$ & 350 & 18.71 & -0.46 & -0.41 & 10.2278 & 9.9023 & -0.43 \\
\hline $3 / 1 / 85$ & 354 & 18.81 & -0.45 & -0.39 & 10.2293 & 9.9037 & -0.42 \\
\hline $3 / 4 / 85$ & 357 & 18.89 & -0.46 & -0.41 & 10.2277 & 9.9037 & -0.42 \\
\hline $3 / 8 / 85$ & 260 & 18.97 & -0.45 & -0.40 & 10.2288 & 9.9028 & -0.43 \\
\hline $3 / 11 / 85$ & 363 & 19.05 & -0.47 & -0.43 & 10.2269 & 9.9002 & -0.45 \\
\hline $3 / 15 / 85$ & 367 & 19.16 & -0.45 & -0.40 & 10.2285 & 9.9026 & -0.43 \\
\hline $3 / 18 / 85$ & 370 & 19.24 & -0.47 & -0.41 & 10.2274 & 9.9022 & -0.44 \\
\hline $3 / 22 / 85$ & 374 & 19.34 & -0.47 & -0.40 & 10.227 & 9.9028 & -0.44 \\
\hline $3 / 25 / 85$ & 377 & 19.42 & -0.46 & -0.41 & 10.2278 & 9.9018 & -0.44 \\
\hline $3 / 29 / 85$ & 381 & 19.52 & -0.43 & -0.38 & 10.2312 & 9.9051 & -0.40 \\
\hline $4 / 1 / 85$ & 384 & 19.60 & -0.45 & -0.39 & 10.2289 & 9.9036 & -0.42 \\
\hline $4 / 8 / 85$ & 391 & 19.77 & -0.44 & -0.39 & 10.23 & 9.9044 & -0.41 \\
\hline $4 / 12 / 85$ & 395 & 19.87 & -0.46 & -0.40 & 10.2284 & 9.9028 & -0.43 \\
\hline $4 / 15 / 85$ & 398 & 19.95 & -0.45 & -0.41 & 10.2289 & 9.9018 & -0.43 \\
\hline $4 / 19 / 85$ & 402 & 20.05 & -0.45 & -0.41 & 10.2291 & 9.9021 & -0.43 \\
\hline $4 / 22 / 85$ & 405 & 20.12 & -0.43 & -0.38 & 10.2308 & 9.9054 & -0.40 \\
\hline $4 / 26 / 85$ & 409 & 20.22 & -0.45 & -0.40 & 10.2287 & 9.903 & -0.43 \\
\hline $4 / 29 / 85$ & 412 & 20.30 & -0.44 & -0.38 & 10.2297 & 9.9045 & -0.41 \\
\hline $5 / 3 / 85$ & 416 & 20.40 & -0.44 & -0.39 & 10.2304 & 9.9039 & -0.41 \\
\hline $5 / 6 / 85$ & 419 & 20.47 & -0.44 & -0.39 & 10.23 & 9.9039 & -0.42 \\
\hline $5 / 10 / 85$ & 423 & 20.57 & -0.44 & -0.39 & 10.2299 & 9.9042 & -0.41 \\
\hline $5 / 13 / 85$ & 426 & 20.64 & -0.44 & -0.40 & 10.2295 & 9.9031 & -0.42 \\
\hline $5 / 17 / 85$ & 430 & 20.74 & -0.44 & -0.39 & 10.23 & 9.9039 & -0.42 \\
\hline $5 / 20 / 85$ & 433 & 20.81 & -0.38 & -0.34 & 10.236 & 9.9088 & -0.36 \\
\hline $5 / 24 / 85$ & 437 & 20.90 & -0.40 & -0.36 & 10.2341 & 9.9069 & -0.38 \\
\hline $5 / 31 / 85$ & 444 & 21.07 & -0.42 & -0.37 & 10.2321 & 9.9064 & -0.39 \\
\hline $6 / 3 / 85$ & 447 & 21.14 & -0.43 & -0.38 & 10.2313 & 9.905 & -0.40 \\
\hline $6 / 17 / 85$ & 461 & 21.47 & -0.41 & -0.36 & 10.233 & 9.9072 & -0.38 \\
\hline
\end{tabular}


TABLE V MOISTURE DESORPTION OF HORIZONTAL STABILIZER S/N B-157-00009, BUTTLINES 8-9

\begin{tabular}{|c|c|c|c|c|c|c|c|}
\hline $\begin{array}{c}\text { DATE } \\
\text { OF } \\
\text { WEIGHING }\end{array}$ & DAYS & $\begin{array}{l}\text { SQRT } \\
\text { OF } \\
\text { TIME }\end{array}$ & $\begin{array}{l}\% \text { MOIST } \\
\text { DESORBED } \\
\text { COUP } 89 B\end{array}$ & $\begin{array}{l}\% \text { MOIST } \\
\text { DESORBED } \\
\text { COUP } 89 T\end{array}$ & $\begin{array}{l}\text { WEIGHT } \\
\text { OF } \\
\text { COUP } 89 \mathrm{~B}\end{array}$ & $\begin{array}{l}\text { WEIGHT } \\
\text { OF } \\
\text { COUP } 89 \mathrm{~T}\end{array}$ & $\begin{array}{l}\text { AVERAGE } \\
\% \text { MOIST } \\
\text { DESORBED }\end{array}$ \\
\hline $3 / 12 / 84$ & 0 & 0 & 0 & 0 & 12.5117 & 8.0043 & 0 \\
\hline $3 / 14 / 84$ & 2 & 1.41 & -0.11 & -0.09 & 12.4978 & 7.9971 & -0.10 \\
\hline $3 / 16 / 84$ & 4 & 2.00 & -0.15 & -0.12 & 12.4933 & 7.9943 & -0.14 \\
\hline $3 / 19 / 84$ & 7 & 2.65 & -0.19 & -0.20 & 12.4883 & 7.9886 & -0.19 \\
\hline $3 / 21 / 84$ & 9 & 3.00 & -0.17 & -0.14 & 12.4907 & 7.9927 & -0.16 \\
\hline $3 / 23 / 84$ & 11 & 3.32 & -0.20 & -0.18 & 12.4863 & 7.99 & -0.19 \\
\hline $3 / 28 / 84$ & 16 & 4.00 & -0.25 & -0.20 & 12.4801 & 7.988 & -0.23 \\
\hline $3 / 30 / 84$ & 18 & 4.24 & -0.24 & -0.20 & 12.4818 & 7.988 & -0.22 \\
\hline $4 / 2 / 84$ & 21 & 4.58 & -0.24 & -0.21 & 12.4816 & 7.9874 & -0.23 \\
\hline $4 / 4 / 84$ & 23 & 4.80 & -0.26 & -0.23 & 12.4795 & 7.9862 & -0.24 \\
\hline $4 / 6 / 84$ & 25 & 5.00 & -0.25 & -0.23 & 12.4798 & 7.9862 & -0.24 \\
\hline $4 / 9 / 84$ & 28 & 5.29 & -0.30 & -0.24 & 12.4746 & 7.9853 & -0.27 \\
\hline $4 / 11 / 84$ & 30 & 5.48 & -0.30 & -0.28 & 12.4745 & 7.9815 & -0.29 \\
\hline $4 / 13 / 84$ & 32 & 5.66 & -0.28 & -0.25 & 12.4767 & 7.9845 & -0.26 \\
\hline $4 / 16 / 84$ & 35 & 5.92 & -0.29 & -0.26 & 12.4759 & 7.9831 & -0.28 \\
\hline $4 / 23 / 84$ & 42 & 6.48 & -0.31 & -0.27 & 12.4725 & 9.9827 & -0.29 \\
\hline $4 / 27 / 84$ & 46 & 6.78 & -0.31 & -0.28 & 12.4732 & 7.9815 & -0.30 \\
\hline $4 / 30 / 84$ & 49 & 7.00 & -0.32 & -0.29 & 12.4719 & 7.981 & -0.30 \\
\hline $5 / 4 / 84$ & 53 & 7.28 & -0.32 & -0.29 & 12.4715 & 7.9813 & -0.30 \\
\hline $5 / 7 / 84$ & 56 & 7.48 & -0.31 & -0.30 & 12.4723 & 7.9804 & -0.31 \\
\hline $5 / 11 / 84$ & 60 & 7.75 & -0.35 & -0.32 & 12.4678 & 7.9788 & -0.33 \\
\hline $5 / 14 / 84$ & 63 & 7.94 & -0.33 & -0.33 & 12.4701 & 7.9778 & -0.33 \\
\hline $5 / 21 / 84$ & 70 & 8.37 & -0.35 & -0.34 & 12.4682 & 7.9768 & -0.35 \\
\hline $5 / 25 / 84$ & 74 & 8.60 & -0.33 & -0.30 & 12.471 & 7.98 & -0.31 \\
\hline $6 / 18 / 84$ & 98 & 9.90 & -0.32 & -0.30 & 12.4713 & 7.9806 & -0.31 \\
\hline $6 / 22 / 84$ & 102 & 10.10 & -0.33 & -0.31 & 12.4704 & 7.9896 & -0.32 \\
\hline $6 / 26 / 84$ & 106 & 10.30 & -0.33 & -0.30 & 12.4707 & 7.98 & -0.32 \\
\hline $6 / 28 / 84$ & 108 & 10.39 & -0.36 & -0.32 & 12.4669 & 7.9783 & -0.34 \\
\hline $7 / 2 / 84$ & 112 & 10.58 & -0.37 & -0.33 & 12.466 & 7.9779 & -0.35 \\
\hline $7 / 9 / 84$ & 119 & 10.91 & -0.36 & -0.32 & 12.4669 & 7.9785 & -0.34 \\
\hline $7 / 13 / 84$ & 123 & 11.09 & -0.37 & -0.34 & 12.4654 & 7.9771 & -0.35 \\
\hline $7 / 16 / 84$ & 126 & 11.22 & -0.36 & -0.33 & 12.4665 & 7.9776 & -0.35 \\
\hline $7 / 18 / 84$ & 128 & 11.31 & -0.36 & -0.33 & 12.4672 & 7.9776 & -0.34 \\
\hline $7 / 20 / 84$ & 130 & 11.40 & -0.36 & -0.34 & 12.4664 & 7.9772 & -0.35 \\
\hline $7 / 23 / 84$ & 133 & 11.53 & -0.35 & -0.33 & 12.4678 & 7.9778 & -0.34 \\
\hline $7 / 27 / 84$ & 137 & 11.70 & -0.35 & -0.33 & 12.4676 & 7.9775 & -0.34 \\
\hline $7 / 30 / 84$ & 140 & 11.83 & -0.33 & -0.32 & 12.4705 & 7.9787 & -0.32 \\
\hline $8 / 3 / 84$ & 144 & 12.00 & -0.35 & -0.33 & 12.4676 & 7.9779 & -0.34 \\
\hline $8 / 6 / 84$ & 147 & 12.12 & -0.35 & -0.33 & 12.4678 & 7.9782 & -0.34 \\
\hline $8 / 10 / 84$ & 151 & 12.29 & -0.35 & -0.33 & 12.4683 & 7.9782 & -0.34 \\
\hline $8 / 13 / 84$ & 154 & 12.41 & -0.34 & -0.31 & 12.4696 & 7.9793 & -0.32 \\
\hline $8 / 17 / 84$ & 158 & 12.57 & -0.36 & -0.35 & 12.4666 & 7.9761 & -0.36 \\
\hline
\end{tabular}


TABLE V MOISTURE DESORPTION OF HORIZONTAL STABILIZER S/N B-157-00009, BUTTLINES 8-9

\begin{tabular}{|c|c|c|c|c|c|c|c|}
\hline $\begin{array}{c}\text { DATE } \\
\text { OF } \\
\text { WEIGHING }\end{array}$ & DAYS & $\begin{array}{c}\text { SQRT } \\
\text { OF } \\
\text { TIME }\end{array}$ & $\begin{array}{l}\% \text { MOIST } \\
\text { DESORBED } \\
\text { COUP } 89 \mathrm{~B}\end{array}$ & $\begin{array}{l}\% \text { MOIST } \\
\text { DESORBED } \\
\text { COUP } 89 T\end{array}$ & $\begin{array}{l}\text { WEIGHT } \\
\text { OF } \\
\text { COUP } 89 \mathrm{~B}\end{array}$ & $\begin{array}{l}\text { WEIGHT } \\
\text { OF } \\
\text { COUP } 89 \mathrm{~T}\end{array}$ & $\begin{array}{l}\text { AVERAGE } \\
\% \text { MOIST } \\
\text { DESORBED }\end{array}$ \\
\hline $8 / 20 / 84$ & 161 & 12.69 & -0.36 & -0.33 & 12.4663 & 7.9775 & -0.35 \\
\hline $8 / 24 / 84$ & 165 & 12.85 & -0.36 & -0.35 & 12.4669 & 7.9762 & -0.35 \\
\hline $8 / 27 / 84$ & 168 & 12.96 & -0.35 & -0.33 & 12.4681 & 7.9778 & -0.34 \\
\hline $8 / 31 / 84$ & 172 & 13.11 & -0.36 & -0.23 & 12.4671 & 7.9774 & -0.35 \\
\hline $9 / 7 / 84$ & 179 & 13.38 & -0.38 & -0.35 & 12.4643 & 7.976 & -0.37 \\
\hline $9 / 10 / 84$ & 182 & 13.49 & -0.36 & -0.35 & 12.4661 & 7.976 & -0.36 \\
\hline $9 / 14 / 84$ & 186 & 13.64 & -0.35 & -0.33 & 12.4679 & 7.9778 & -0.34 \\
\hline $9 / 17 / 84$ & 189 & 13.75 & -0.38 & -0.37 & 12.4642 & 7.9748 & -0.37 \\
\hline $9 / 21 / 84$ & 193 & 13.89 & -0.38 & -0.37 & 12.4638 & 7.9739 & -0.38 \\
\hline $9 / 24 / 84$ & 196 & 14.00 & -0.36 & -0.34 & 12.4667 & 7.9773 & -0.35 \\
\hline $9 / 28 / 84$ & 200 & 14.14 & -0.38 & -0.36 & 12.464 & 7.9752 & -0.37 \\
\hline $10 / 1 / 84$ & 203 & 14.25 & -0.38 & -0.36 & 12.4636 & 7.9751 & -0.37 \\
\hline $10 / 5 / 84$ & 207 & 14.39 & -0.38 & -0.36 & 12.4639 & 7.9752 & -0.37 \\
\hline $10 / 8 / 84$ & 210 & 14.49 & -0.39 & -0.37 & 12.4631 & 7.975 & -0.38 \\
\hline $10 / 12 / 84$ & 214 & 14.63 & -0.39 & -0.39 & 12.4624 & 7.9732 & -0.39 \\
\hline $10 / 15 / 84$ & 217 & 14.73 & -0.30 & -0.38 & 12.4618 & 7.974 & -0.39 \\
\hline $10 / 19 / 84$ & 221 & 14.87 & -0.39 & -0.38 & 12.4629 & 7.9738 & -0.39 \\
\hline $10 / 22 / 84$ & 224 & 14.97 & -0.38 & -0.38 & 12.4637 & 7.9738 & -0.38 \\
\hline $10 / 26 / 84$ & 228 & 15.10 & -0.37 & -0.36 & 12.4654 & 7.9756 & -0.36 \\
\hline $10 / 29 / 84$ & 231 & 15.20 & -0.37 & -0.36 & 12.4659 & 7.9754 & -0.36 \\
\hline $11 / 5 / 84$ & 238 & 15.43 & -0.37 & -0.35 & 12.4654 & 7.9766 & -0.36 \\
\hline $11 / 9 / 84$ & 242 & 15.56 & -0.38 & -0.36 & 12.4638 & 7.9751 & -0.37 \\
\hline $11 / 12 / 84$ & 245 & 15.65 & -0.36 & -0.33 & 12.467 & 7.9775 & -0.35 \\
\hline $11 / 16 / 84$ & 249 & 15.78 & -0.38 & -0.36 & 12.4643 & 7.9755 & -0.37 \\
\hline $11 / 19 / 84$ & 252 & 15.87 & -0.30 & -0.38 & 12.4617 & 7.9737 & -0.39 \\
\hline $11 / 26 / 84$ & 259 & 16.09 & -0.39 & -0.36 & 12.4629 & 7.9755 & -0.37 \\
\hline $11 / 30 / 84$ & 263 & 16.22 & -0.30 & -0.37 & 12.4621 & 7.9745 & -0.38 \\
\hline $12 / 7 / 84$ & 270 & 16.43 & -0.42 & -0.40 & 12.4591 & 7.9721 & -0.41 \\
\hline $12 / 10 / 84$ & 273 & 16.52 & -0.41 & -0.40 & 12.4605 & 7.9722 & -0.41 \\
\hline $12 / 14 / 84$ & 277 & 16.64 & -0.40 & -0.39 & 12.4618 & 7.973 & -0.39 \\
\hline $12 / 17 / 84$ & 280 & 16.73 & -0.39 & -0.38 & 12.4623 & 7.9736 & -0.39 \\
\hline $1 / 4 / 85$ & 298 & 17.26 & -0.43 & -0.41 & 12.5483 & 7.9712 & -0.42 \\
\hline $1 / 7 / 85$ & 301 & 17.35 & -0.43 & -0.42 & 12.4581 & 7.9706 & -0.42 \\
\hline $1 / 11 / 85$ & 305 & 17.46 & -0.44 & -0.39 & 12.4565 & 7.9728 & -0.42 \\
\hline $1 / 18 / 85$ & 312 & 17.66 & -0.45 & -0.43 & 12.4552 & 7.9702 & -0.44 \\
\hline $1 / 21 / 85$ & 315 & 17.75 & -0.46 & -0.42 & 12.4542 & 7.9706 & -0.44 \\
\hline $1 / 25 / 85$ & 319 & 17.86 & -0.43 & -0.43 & 12.5673 & 7.9699 & -0.43 \\
\hline $1 / 28 / 85$ & 322 & 17.94 & -0.44 & -0.41 & 12.4567 & 7.9715 & -0.42 \\
\hline $2 / 1 / 85$ & 236 & 18.06 & -0.43 & -0.41 & 12.4574 & 7.9717 & -0.42 \\
\hline $2 / 4 / 85$ & 239 & 18.14 & -0.45 & -0.41 & 12.4555 & 7.9711 & -0.43 \\
\hline $2 / 8 / 85$ & 333 & 18.25 & -0.44 & -0.43 & 12.457 & 7.9696 & -0.44 \\
\hline $2 / 11 / 85$ & 336 & 18.33 & -0.45 & -0.43 & 12.4551 & 7.9696 & -0.44 \\
\hline $2 / 15 / 85$ & 340 & 18.44 & -0.47 & -0.43 & 12.4535 & 7.9702 & -0.45 \\
\hline
\end{tabular}


TABLE V MOISTURE DESORPTION OF HORIZONTAL STABILIZER S/N B-157-00009, BUTTLINES 8-9

\begin{tabular}{|c|c|c|c|c|c|c|c|}
\hline $\begin{array}{c}\text { DATE } \\
\text { OF } \\
\text { WEIGHING }\end{array}$ & DAYS & $\begin{array}{l}\text { SQRT } \\
\text { OF } \\
\text { TIME }\end{array}$ & $\begin{array}{l}\% \text { MOIST } \\
\text { DESORBED } \\
\text { COUP } 89 B\end{array}$ & $\begin{array}{l}\% \text { MOIST } \\
\text { DESORBED } \\
\text { COUP } 89 T\end{array}$ & $\begin{array}{l}\text { WEIGHT } \\
\text { OF } \\
\text { COUP } 89 \mathrm{~B}\end{array}$ & $\begin{array}{l}\text { WE IGHT } \\
\text { OF } \\
\text { COUP } 89 \mathrm{~T}\end{array}$ & $\begin{array}{l}\text { AVERAGE } \\
\% \text { MOIST } \\
\text { DESORBED }\end{array}$ \\
\hline $2 / 18 / 85$ & 343 & 18.52 & -0.47 & -0.45 & 12.4527 & 7.9683 & -0.46 \\
\hline $2 / 22 / 85$ & 347 & 18.63 & -0.46 & -0.44 & 12.4543 & 7.9691 & -0.45 \\
\hline $2 / 25 / 85$ & 350 & 18.71 & -0.46 & -0.43 & 12.4537 & 7.9701 & -0.45 \\
\hline $3 / 1 / 85$ & 354 & 18.81 & -0.45 & -0.42 & 12.4554 & 7.971 & -0.43 \\
\hline $3 / 4 / 85$ & 357 & 18.89 & -0.47 & -0.45 & 12.4529 & 7.9685 & -0.46 \\
\hline $3 / 8 / 85$ & 360 & 18.97 & -0.45 & -0.43 & 12.455 & 7.97 & -0.44 \\
\hline $3 / 11 / 85$ & 363 & 19.05 & -0.48 & -0.47 & 12.4519 & 7.967 & -0.47 \\
\hline $3 / 15 / 85$ & 267 & 19.16 & -0.45 & -0.42 & 12.4554 & 7.9704 & -0.44 \\
\hline $3 / 18 / 85$ & 270 & 19.24 & -0.46 & -0.45 & 12.4544 & 7.9679 & -0.46 \\
\hline $3 / 22 / 85$ & 374 & 19.34 & -0.46 & -0.46 & 12.4537 & 7.9678 & -0.46 \\
\hline $3 / 25 / 85$ & 377 & 19.42 & -0.46 & -0.44 & 12.4538 & 7.9694 & -0.45 \\
\hline $3 / 29 / 85$ & 381 & 19.52 & -0.42 & -0.40 & 12.4587 & 7.89722 & -0.41 \\
\hline $4 / 1 / 85$ & 384 & 19.60 & -0.45 & -0.42 & 12.4557 & 7.9708 & -0.43 \\
\hline $4 / 8 / 85$ & 391 & 19.77 & -0.47 & -0.42 & 12.4533 & 7.9705 & -0.44 \\
\hline $4 / 12 / 85$ & 395 & 19.87 & -0.46 & -0.43 & 12.4546 & 7.9699 & -0.44 \\
\hline $4 / 15 / 85$ & 398 & 19.95 & -0.46 & -0.42 & 12.4544 & 7.9706 & -0.44 \\
\hline $4 / 19 / 85$ & 402 & 20.05 & -0.46 & -0.43 & 12.4537 & 7.9701 & -0.45 \\
\hline $4 / 22 / 85$ & 405 & 20.12 & -0.43 & -0.41 & 12.4577 & 7.9712 & -0.42 \\
\hline $4 / 26 / 85$ & 409 & 20.22 & -0.45 & -0.44 & 12.4553 & 7.9693 & -0.44 \\
\hline $4 / 29 / 85$ & 412 & 20.30 & -0.44 & -0.42 & 12.4572 & 7.9705 & -0.43 \\
\hline $5 / 3 / 85$ & 416 & 20.40 & -0.43 & -0.41 & 12.4573 & 7.9717 & -0.42 \\
\hline $5 / 6 / 85$ & 419 & 20.47 & -0.44 & -0.41 & 12.4571 & 7.9715 & -0.42 \\
\hline $5 / 10 / 85$ & 423 & 20.57 & -0.44 & -0.42 & 12.4563 & 7.9808 & -0.43 \\
\hline $5 / 13 / 85$ & 426 & 20.64 & -0.43 & -0.42 & 12.4583 & 7.9708 & -0.42 \\
\hline $5 / 17 / 85$ & 430 & 20.74 & -0.42 & -0.41 & 12.4587 & 7.9715 & -0.42 \\
\hline $5 / 20 / 85$ & 433 & 20.81 & -0.39 & -0.37 & 12.4624 & 7.9743 & -0.38 \\
\hline $5 / 24 / 85$ & 437 & 20.90 & -0.42 & -0.39 & 12.4594 & 7.9732 & -0.40 \\
\hline $5 / 31 / 85$ & 444 & 21.07 & -0.42 & -0.40 & 12.4597 & 7.9723 & -0.41 \\
\hline $6 / 3 / 85$ & 447 & 21.14 & -0.43 & -0.41 & 12.4583 & 7.9713 & -0.42 \\
\hline $6 / 17 / 85$ & 461 & 21.47 & -0.43 & -0.39 & 12.4584 & 7.9729 & -0.41 \\
\hline
\end{tabular}




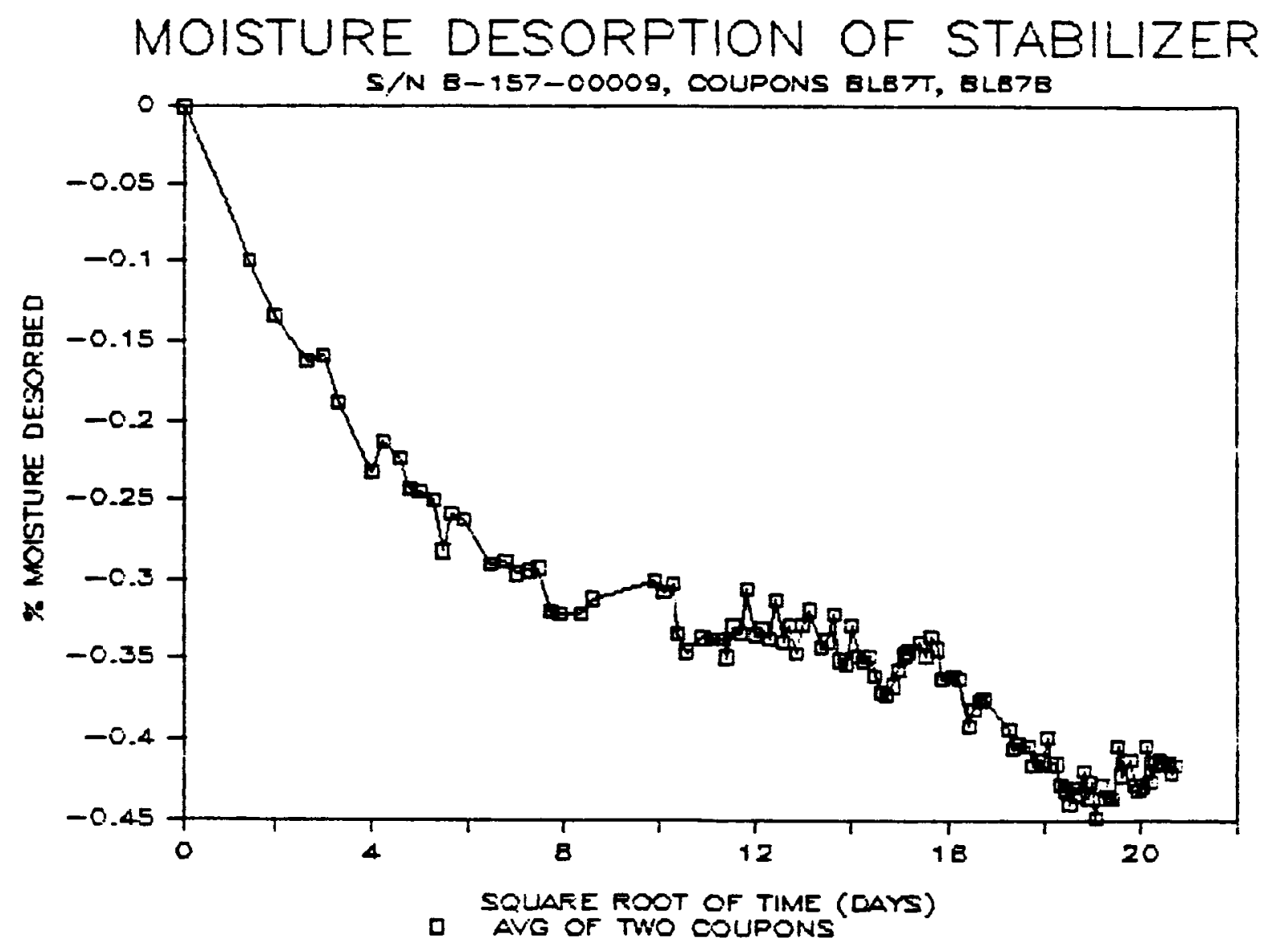

FIGURE 4

MOISTURE DESORPTION OF STABILIZER S/N B-157-00009

COUPONS BL67T, BL67B 
$3.1 .2 \quad \underline{S} / \mathrm{N} \mathrm{B}-157-00021$

Horizontal stabilizer S/N B-157-00021 was returned from the field for full scale static and small scale coupon testing. After 66 months of in-service environmental exposure, the stabilizer had accumulated 4213 flight hours. The environmental history of the stabilizer is detailed in Table VI.

The horizontal stabilizer was proof load deflection tested in accordance with the aforementioned procedure required for production acceptance. An acceptable deflection of $3.86 \mathrm{~mm}$ (.150 in), indicating no loss of stiffness after 66 months in service, was noted.

Visual inspection and coin tapping revealed two small areas of disbond in the torque box section. One disbond measured approximately $3 / 4$ inch high by $1-1 / 2$ inch wide and was located at left Buttline 3. The other disbond measured approximately 1 inch high by 3 inches wide, located at right Buttline 3 . Damage was thought to have been sustained during removal of the stabilizer from the aircraft.

Although the stabilizer was scheduled for full scale static testing, concern over the disbond prompted $\mathrm{S}-76 \mathrm{~B}$ engineers to verbally request permission from NASA representatives to test the stabilizer in fatigue. With the approval of the NASA technical monitor, the stabilizer was first static tested to the design limit load, then tested in fatigue.

The stabilizer was statically loaded as detailed in Figure 5 . As the design limit load is asymmetrical, the loads shown in Figure 5 are designated $L$ for left side and $R$ for right side of the stabilizer. To allow for direct comparison with the baseline, the static test was conducted at $160^{\circ} \mathrm{F}$.

When no fracture occurred, the stabilizer was to be fatigue tested with the loads detailed in Figure 3. Owing to an error in loading, the fatigue loads applied were 23 percent higher than the baseline loads of Figure 3. During fatigue testing, the stabilizer disbonded from the test fixture. Proof load deflection tests were run to insure that fracture did not occur in the stabilizer as well. The stabilizer was then rebonded into the test fixture, and the test continued. Testing was stopped at 59,980 cycles, when test engineers visually observed a fracture in the torque box. While a complete teardown analysis is in progress, preliminary results indicate that the fractured area was located between Buttline $3 \mathrm{~L}$ and Buttline $3 R$, as anticipated.

Six graphite/epoxy coupons were removed from the failed stabilizer at Buttlines 4-9, for moisture desorption. Two Kevlar/epoxy coupons were also removed from Buttlines 4-5. Desorption of the coupons is in progress. 
Previously in the program, stabilizer S/N B-157-00076 having 17 months and 1600 flight hours, was returned from the field for full scale static tests. Details of the testing were documented in the first annual report, Reference (1). For comparison purposes, a summary of proof load deflection testing, flight hours and moisture level (B-157-00021 in progress) for all three stabilizers is presented in Figure 6. 
TABLE VI

STABILIZER S/N B-157-00021

SUMMARY OF ENVIRONMENTAL HISTORY

\begin{tabular}{|c|c|c|c|c|}
\hline \multicolumn{2}{|r|}{ DATE } & \multicolumn{2}{|c|}{$\begin{array}{c}\text { AVERAGE } \\
\text { TEMPERATURE }\end{array}$} & $\begin{array}{c}\text { AVERAGE } \\
\text { RELATIVE HUMIDITY } \\
(\%)\end{array}$ \\
\hline $10 / 23 / 79$ & $-10 / 31 / 79$ & 20.5 & 68.9 & 79.0 \\
\hline $11 / 01 / 79$ & $-11 / 30 / 79$ & 12.4 & 54.5 & 75.4 \\
\hline $12 / 01 / 79$ & $-12 / 31 / 79$ & 10.3 & 50.5 & 78.1 \\
\hline $1 / 01 / 80$ & $-1 / 31 / 80$ & 11.9 & 53.4 & 86.4 \\
\hline $2 / 01 / 80$ & $-\quad 2 / 29 / 80$ & 10.3 & 50.6 & 80.5 \\
\hline $3 / 01 / 80$ & $-3 / 31 / 80$ & 15.2 & 59.4 & 81.4 \\
\hline $4 / 01 / 80$ & $-\quad 4 / 30 / 80$ & 18.4 & 65.1 & 76.5 \\
\hline $5 / 01 / 80$ & $-5 / 31 / 80$ & 23.8 & 74.8 & 83.9 \\
\hline $6 / 01 / 80$ & $-6 / 30 / 80$ & 27.1 & 80.8 & 80.3 \\
\hline $7 / 01 / 80$ & $-7 / 31 / 80$ & 28.2 & 82.8 & 72.5 \\
\hline $8 / 01 / 80$ & $-8 / 31 / 80$ & 27.4 & 81.3 & 74.0 \\
\hline $9 / 01 / 80$ & $-9 / 30 / 80$ & 26.3 & 79.4 & 79.3 \\
\hline $10 / 01 / 80$ & $-10 / 31 / 80$ & 18.0 & 64.4 & 69.8 \\
\hline $11 / 01 / 80$ & $-11 / 30 / 80$ & 12.7 & 54.8 & 78.0 \\
\hline $12 / 01 / 80$ & $-12 / 31 / 80$ & 10.7 & 51.3 & 75.0 \\
\hline $1 / 01 / 81$ & $-1 / 31 / 81$ & 8.2 & 46.8 & 73.5 \\
\hline $2 / 01 / 81$ & $-2 / 28 / 81$ & 11.1 & 52.0 & 74.0 \\
\hline $3 / 01 / 81$ & $-3 / 31 / 81$ & 14.9 & 58.9 & 66.4 \\
\hline $4 / 01 / 81$ & $-4 / 30 / 81$ & 21.4 & 70.5 & 76.1 \\
\hline $5 / 01 / 81$ & $-5 / 31 / 81$ & 22.6 & 72.6 & 73.3 \\
\hline $6 / 01 / 81$ & $-6 / 30 / 81$ & 26.8 & 80.3 & 82.1 \\
\hline $7 / 01 / 81$ & $-7 / 31 / 81$ & 27.3 & 81.1 & 81.8 \\
\hline $8 / 01 / 81$ & $-8 / 31 / 81$ & 26.9 & 80.5 & 79.3 \\
\hline $9 / 01 / 81$ & $-9 / 30 / 81$ & 23.8 & 74.8 & 77.3 \\
\hline $10 / 01 / 81$ & $-10 / 31 / 81$ & 20.1 & 68.1 & 79.1 \\
\hline $11 / 01 / 81$ & $-11 / 30 / 81$ & 16.1 & 60.9 & 80.9 \\
\hline $12 / 01 / 81$ & $-12 / 31 / 81$ & 11.4 & 52.5 & 73.4 \\
\hline $1 / 01 / 82$ & $-1 / 31 / 82$ & 11.1 & 51.9 & 76.9 \\
\hline $2 / 01 / 82$ & $-2 / 28 / 82$ & 10.8 & 51.4 & 78.4 \\
\hline $3 / 01 / 82$ & $-3 / 31 / 82$ & 16.9 & 62.5 & 82.6 \\
\hline $4 / 01 / 82$ & $-4 / 30 / 82$ & 18.9 & 66.1 & 80.1 \\
\hline $5 / 01 / 82$ & $-5 / 31 / 82$ & 23.2 & 73.8 & 82.1 \\
\hline $6 / 01 / 82$ & $-6 / 30 / 82$ & 26.4 & 79.6 & 82.4 \\
\hline
\end{tabular}


TABLE VI (Continued)

STABILIZER S/N B-157-0.0021

SUMMARY OF ENVIRONMENTAL HISTORY

\begin{tabular}{|c|c|c|c|c|}
\hline \multirow{2}{*}{\multicolumn{2}{|c|}{ DATE }} & \multicolumn{2}{|c|}{$\begin{array}{c}\text { AVERAGE } \\
\text { TEMPERATURE }\end{array}$} & \multirow{2}{*}{$\begin{array}{c}\text { AVERAGE } \\
\text { RELATIVE HUMIDITY } \\
(\%)\end{array}$} \\
\hline & & $\left({ }^{\circ} \mathrm{C}\right)$ & $\left({ }^{\circ} \mathrm{F}\right)$ & \\
\hline $7 / 01 / 82$ & $-7 / 31 / 82$ & 27.2 & 80.9 & 80.8 \\
\hline $8 / 01 / 82$ & $-\quad 8 / 31 / 82$ & 26.9 & 80.5 & 78.8 \\
\hline $9 / 01 / 82$ & $-\quad 9 / 30 / 82$ & 24.2 & 75.6 & 75.5 \\
\hline $10 / 01 / 82$ & $-10 / 31 / 82$ & 20.2 & 68.3 & 70.9 \\
\hline $11 / 01 / 82$ & $-11 / 30 / 82$ & 16.4 & 61.5 & 74.3 \\
\hline $12 / 01 / 82$ & $-12 / 31 / 82$ & 13.9 & 57.0 & 81.1 \\
\hline $1 / 01 / 83$ & $-1 / 31 / 83$ & 9.5 & 49.1 & 81.1 \\
\hline $2 / 01 / 83$ & $-\quad 2 / 28 / 83$ & 11.3 & 52.4 & 77.3 \\
\hline $3 / 01 / 83$ & $-\quad 3 / 31 / 83$ & 14.2 & 57.6 & 73.5 \\
\hline $4 / 01 / 83$ & $-\quad 4 / 30 / 83$ & 17.5 & 63.5 & 73.4 \\
\hline $5 / 01 / 83$ & $-5 / 31 / 83$ & 23.0 & 73.4 & 77.1 \\
\hline $6 / 01 / 83$ & $-6 / 30 / 83$ & 25.6 & 78.0 & 81.3 \\
\hline $7 / 01 / 83$ & $-7 / 31 / 83$ & 28.2 & 82.8 & 78.1 \\
\hline $8 / 01 / 83$ & $-\quad 8 / 31 / 83$ & 27.9 & 82.1 & 81.4 \\
\hline $9 / 01 / 83$ & $-9 / 30 / 83$ & 24.2 & 75.6 & 77.9 \\
\hline $10 / 01 / 83$ & $-10 / 31 / 83$ & 21.1 & 69.9 & 73.3 \\
\hline $11 / 01 / 83$ & $-11 / 30 / 83$ & 16.7 & 62.1 & 75.8 \\
\hline $12 / 01 / 83$ & $-12 / 31 / 83$ & 9.1 & 48.3 & 73.3 \\
\hline $1 / 01 / 84$ & $-\quad 1 / 31 / 84$ & 8.9 & 48.1 & 74.3 \\
\hline $2 / 01 / 84$ & $-\quad 2 / 29 / 84$ & 13.3 & 55.9 & 68.1 \\
\hline $3 / 01 / 84$ & $-\quad 3 / 31 / 84$ & 16.9 & 62.4 & 72.5 \\
\hline $4 / 01 / 84$ & $-\quad 4 / 30 / 84$ & 21.1 & 69.9 & 66.9 \\
\hline $5 / 01 / 84$ & $-\quad 5 / 31 / 84$ & 23.9 & 75.0 & 72.3 \\
\hline $6 / 01 / 84$ & $-6 / 30 / 84$ & 26.4 & 79.5 & 79.0 \\
\hline
\end{tabular}




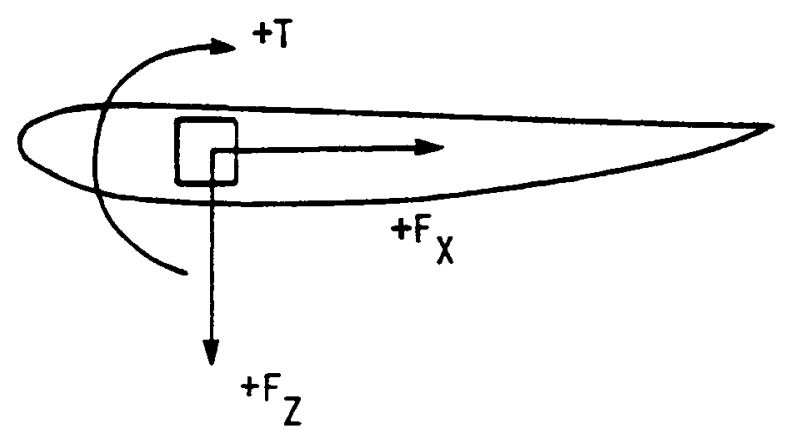

\footnotetext{
$\begin{array}{llllll}\mathrm{F}_{\mathrm{XL}} & -\mathrm{F}_{\mathrm{ZL}} & \mathrm{T}_{\mathrm{L}} & \mathrm{F}_{\mathrm{XR}} & \mathrm{F}_{\mathrm{ZR}} & \mathrm{T}_{\mathrm{R}}\end{array}$

$\begin{array}{llllll}511 \mathrm{~N} & 4257 \mathrm{~N} & 107 \mathrm{~N}-\mathrm{m} & -476 \mathrm{~N} & 196 \mathrm{~N} & 106 \mathrm{~N}-\mathrm{m}\end{array}$

$\begin{array}{lllll}(115 \mathrm{LB}) & (957 \mathrm{LB}) \quad(950 \mathrm{IN}-\mathrm{LB}) & (-107 \mathrm{LB}) & (44 \mathrm{LB}) & (935 \mathrm{IN}-\mathrm{LB})\end{array}$

L, R - LEFT OR RIGHT SIDE LOADS, RESPECTIVELY

FIGURE 5. S-76 STABILIZER LOCATION AND MAGNITUDE OF STATIC TEST LOADS
} 


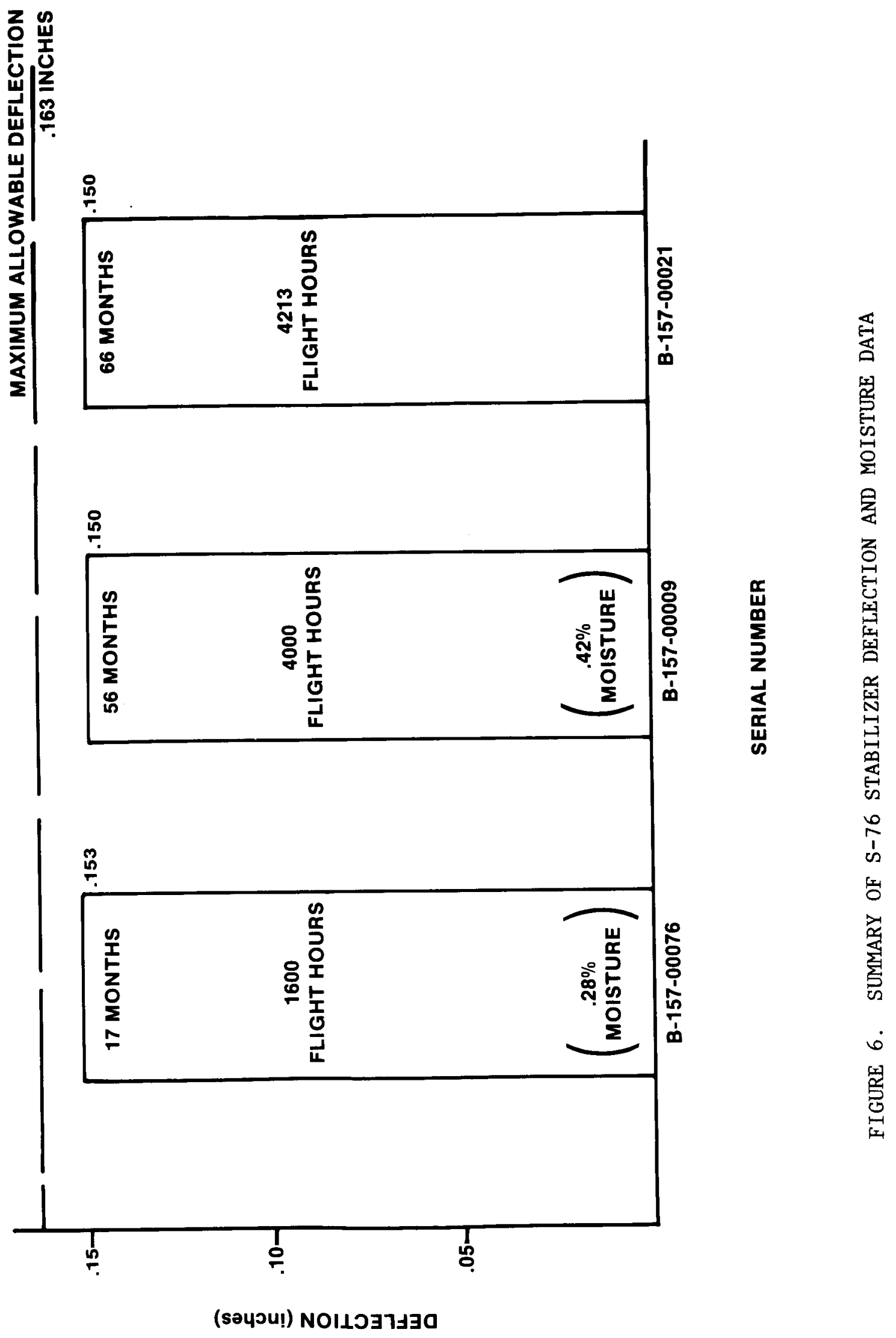


3.2 Tail Rotor Spars

To date, nine tail rotor spars have been returned from the field for evaluation. The tail rotor blade consists of two separable tail rotor paddles attached to one tail rotor spar. After the paddles were removed, the tail rotor spar was non-destructively inspected. No abnormalities were found. Table VII presents the service time, location, fatigue data and measured moisture content of the spars evaluated. As can be seen in Table VII, six of the tail rotor spars returned from the field were full scale fatigue tested. Three of the tail rotor spars were returned for small scale (coupon) testing. During this reporting period, spar S/N A-116-00178 was returned from the field for small scale testing. The environmental history of the spar is summarized in Table VIII. Spar S/N A-116-00178 had accumulated 3753 flight hours after 55 months of in-service exposure.

Coupons were removed from Buttlines 11-12 Right and Buttlines 11-12 Left from S/N A-116-00178 tail rotor spar for static and fatigue testing. As shown in Figure 7, twelve coupons were removed from each side of the spar, six for short beam shear static and six for short beam shear fatigue testing. Of the six static specimens removed from each end, three were tested at room temperature and three at $170^{\circ} \mathrm{F}$, in accordance with ASTM D 2344, Reference (3). Data is compiled in Tables IX and X. Although specimens were marked A or $B$ to designate the end of the spar from which they were removed, application of the $t$ distribution test in accordance with Freund, Reference (4), and as detailed in Figure 8 , showed that the data could be combined. At room temperature, the average interlaminar shear strength generated was $12.6 \mathrm{ksi}$. The average interlaminar shear strength at $170^{\circ} \mathrm{F}$ was $10.2 \mathrm{ksi}$.

For comparison, coupon data generated for tail rotor spar S/N A116-00178 is presented in Tables XI and XII with coupon data from the two tail rotor spars previously returned from the field for coupon testing. Tail rotor spars S/N A-116-00150 and S/N A-116-00283 were both returned from the field after 38 months of in-service exposure. Inspection shows values from spar S/N A-116-00178 to appear high compared to results from the other two spars, however, when compared to 14 ply graphite/epoxy panel data, results are shown to be compatable to expected values.

All coupon fatigue testing was conducted at room temperature. The maximum stress versus cycles to fracture plot generated for spar S/N A-116-00178 is presented in Figure 9. As in the static properties, data compiled in Table XIII shows fatigue properties of tail rotor spar S/N A-116-00178 to be somewhat higher (14 percent) than the properties of the two previously returned spars $\mathrm{S} / \mathrm{N} A-116-00150$ and $\mathrm{S} / \mathrm{N} \mathrm{A}-116-00283$. 
Moisture desorption coupons were removed from Buttlines 5-7 Right and Buttlines 5-7 Left for moisture analysis. An average of 0.60 percent moisture was desorbed from the eight coupons removed. The desorption-time plot for coupon 5-7-1 removed from the right side of the spar is typical and shown in Figure 10. Full results of the spar coupon moisture desorption tests are detailed in Tables XIV and XV.

A moisture-time profile was developed for the tail rotor blades operating in the Louisiana Gulf coast region. Weather data from Lake Charles, Louisiana was used in predicting the expected moisture absorbed. Measured moisture values are shown in Figure 11 on a plot of predicted moisture versus calendar time. The effects of solar radiation are not included in the moisture prediction, as discussed in the earlier reports, References (1) and (2). As can be seen in the Figure, the measured moisture values plotted on the curve of predicted moisture versus calendar time fall within an acceptable range of scatter. In addition, Figure 12 presents the measured versus analysis moisture contents of in-service $S-76$ spars as a one-to-one correlation. Inspection of the plot shows the prediction analysis has been generally accurate, with most variation being conservative. 
TABLE VII. FATIGUE TEST AND DATA SUMMARY FOR TAIL ROTOR SPARS

\begin{tabular}{|c|c|c|c|c|}
\hline $\begin{array}{l}\text { TAIL ROTOR } \\
\text { SPAR S/N }\end{array}$ & $\begin{array}{ll}\text { IN-SERVICE } & \text { TIME } \\
\text { MONTHS/FLT } & \text { HRS }\end{array}$ & $\begin{array}{l}\text { CYCLIC SHEAR } \\
\text { STRESS, PSI }\end{array}$ & $\begin{array}{l}\text { CYCLES TO } \\
\text { CRACK }\end{array}$ & $\begin{array}{l}\text { MOISTURE } \\
\text { CONTENT }(\%)\end{array}$ \\
\hline 00046 & $\begin{array}{l}25 \text { Months } * 1 \\
150 \text { flight hours }\end{array}$ & $\begin{array}{l}\text { (a) } 3980 \\
\text { (b) } 3980\end{array}$ & $\begin{array}{lll}.25 \times 10^{6} \\
.38 \times 10^{6}\end{array}$ & .29 \\
\hline 00064 & $\begin{array}{l}25 \text { Months } * 1 \\
150 \text { flight hours }\end{array}$ & $\begin{array}{l}\text { (a) } 4320 \\
\text { (b) } 4320\end{array}$ & $\begin{array}{l}.035 \times 10^{6} \\
.071 \times 10^{6}\end{array}$ & .32 \\
\hline 00094 & $\begin{array}{l}29 \text { Months } * 2 \\
2390 \text { flight hours }\end{array}$ & $\begin{array}{l}\text { (a) } 3890 \\
\text { (b) } 3920\end{array}$ & $\begin{array}{l}.286 \times 10^{6} \\
.174 \times 10^{6}\end{array}$ & .26 \\
\hline 00283 & $\begin{array}{l}37 \text { Months } * 2 \\
1884 \text { flight hours }\end{array}$ & (coupon tests) & (coupon tests) & .36 \\
\hline 00150 & $\begin{array}{l}37 \text { Months } * 2 \\
2385 \text { flight hours }\end{array}$ & (coupon tests) & (coupon tests) & .40 \\
\hline 00237 & $\begin{array}{l}39 \text { Months } * 2 \\
1596 \text { flight hours }\end{array}$ & 4520 & $.267 \times 10^{6}$ & .47 \\
\hline 00172 & $\begin{array}{l}39 \text { Months } * 2 \\
2533 \text { flight hours }\end{array}$ & 4270 & $.218 \times 10^{6}$ & .49 \\
\hline 00114 & $\begin{array}{l}49 \text { Months } * 2 \\
3350 \text { flight hours }\end{array}$ & 4416 & $.839 \times 10^{6}$ & .56 \\
\hline 00178 & $\begin{array}{l}55 \text { Months } * 2 \\
3753 \text { flight hours }\end{array}$ & (coupon tests) & (coupon tests) & .60 \\
\hline
\end{tabular}

$* 1$ In-service location: West Palm Beach, Florida

$* 2$ In-service location: Gulf Coast Region, Louisiana 
TABLE VIII

SPAR S/N A-116-00178 (PADDLE S/N A-137-00067)

SUMMARY OF ENVIRONMENTAL HISTORY

\begin{tabular}{|c|c|c|c|c|}
\hline \multicolumn{2}{|r|}{ DATE } & \multicolumn{2}{|c|}{$\begin{array}{c}\text { AVERAGE } \\
\text { TEMPERATURE }\end{array}$} & $\begin{array}{c}\text { AVERAGE } \\
\text { RELATIVE HUMIDITY } \\
(\%)\end{array}$ \\
\hline $11 / 08 / 79$ & $-11 / 30 / 79$ & 12.4 & 54.4 & 75.4 \\
\hline $12 / 01 / 79$ & $-12 / 31 / 79$ & 10.3 & 50.5 & 78.1 \\
\hline $1 / 01 / 80$ & $-1 / 31 / 80$ & 11.9 & 33.4 & 86.4 \\
\hline $2 / 01 / 80$ & $-2 / 29 / 80$ & 10.3 & 50.6 & 80.5 \\
\hline $3 / 01 / 80$ & $-3 / 31 / 80$ & 15.2 & 59.4 & 81.4 \\
\hline $4 / 01 / 80$ & $-4 / 30 / 80$ & 18.4 & 65.1 & 76.5 \\
\hline $5 / 01 / 80$ & $-5 / 31 / 80$ & 23.9 & 74.8 & 83.9 \\
\hline $6 / 01 / 80$ & $-6 / 30 / 80$ & 27.1 & 80.8 & 80.3 \\
\hline $7 / 01 / 80$ & $-7 / 31 / 80$ & 28.2 & 82.8 & 72.5 \\
\hline $8 / 01 / 80$ & $-8 / 31 / 80$ & 27.4 & 81.3 & 74.0 \\
\hline $9 / 01 / 80$ & $-9 / 30 / 80$ & 26.3 & 79.4 & 79.3 \\
\hline $10 / 01 / 80$ & $-10 / 31 / 80$ & 18.0 & 64.4 & 69.8 \\
\hline $11 / 01 / 80$ & $-11 / 30 / 80$ & 12.7 & 54.8 & 78.0 \\
\hline $12 / 01 / 80$ & $-12 / 31 / 80$ & 10.7 & 51.3 & 75.0 \\
\hline $1 / 01 / 81$ & $-\quad 1 / 31 / 81$ & 8.2 & 46.8 & 73.5 \\
\hline $2 / 01 / 81$ & $-\quad 2 / 28 / 81$ & 11.1 & 52.0 & 74.0 \\
\hline $3 / 01 / 81$ & $-3 / 31 / 81$ & 14.9 & 58.9 & 66.4 \\
\hline $4 / 01 / 81$ & $-\quad 4 / 30 / 81$ & 21.4 & 70.5 & 76.1 \\
\hline $5 / 01 / 81$ & $-\quad 5 / 31 / 81$ & 22.6 & 72.6 & 73.3 \\
\hline $6 / 01 / 81$ & $-6 / 30 / 81$ & 26.8 & 80.3 & 82.1 \\
\hline $7 / 01 / 81$ & $-7 / 31 / 81$ & 27.3 & 81.1 & 81.8 \\
\hline $8 / 01 / 81$ & $-\quad 8 / 31 / 81$ & 26.9 & 80.5 & 79.3 \\
\hline $9 / 01 / 81$ & $-9 / 30 / 81$ & 23.8 & 74.8 & 77.3 \\
\hline $10 / 01 / 81$ & $-10 / 31 / 81$ & 20.1 & 68.1 & 79.1 \\
\hline $11 / 01 / 81$ & $-11 / 30 / 81$ & 16.1 & 60.9 & 80.9 \\
\hline $12 / 01 / 81$ & $-12 / 31 / 81$ & 11.4 & 52.5 & 73.4 \\
\hline $1 / 01 / 82$ & - $1 / 31 / 82$ & 11.1 & 51.9 & 76.9 \\
\hline $2 / 01 / 82$ & $-2 / 28 / 82$ & 10.8 & 51.4 & 78.4 \\
\hline $3 / 01 / 82$ & $-3 / 31 / 82$ & 16.9 & 62.5 & 82.6 \\
\hline $4 / 01 / 82$ & $-4 / 30 / 82$ & 18.9 & 66.1 & 80.1 \\
\hline $5 / 01 / 82$ & $-5 / 31 / 82$ & 23.2 & 73.8 & 82.1 \\
\hline $6 / 01 / 82$ & $-6 / 30 / 82$ & 26.4 & 79.6 & 82.4 \\
\hline $7 / 01 / 82$ & $-7 / 31 / 82$ & 27.2 & 80.9 & 80.8 \\
\hline
\end{tabular}


TABLE VIII (Continued)

SPAR S/N A-116-00178 (PADDLE S/N A-137-00067)

SUMMARY OF ENVIRONMENTAL HISTORY

\begin{tabular}{|c|c|c|c|c|}
\hline \multicolumn{2}{|c|}{ DATE } & \multicolumn{2}{|c|}{$\begin{array}{c}\text { AVERAGE } \\
\text { TEMPERATURE }\end{array}$} & $\begin{array}{c}\text { AVERAGE } \\
\text { RELATIVE HUMIDITY } \\
(\%)\end{array}$ \\
\hline $8 / 01 / 82$ & $8 / 31 / 82$ & 26.9 & 80.5 & 78.8 \\
\hline $9 / 01 / 82$ & $-\quad 9 / 30 / 82$ & 24.2 & 75.6 & 75.5 \\
\hline $10 / 01 / 82$ & $-10 / 31 / 82$ & 20.2 & 68.3 & 70.9 \\
\hline $11 / 01 / 82$ & $-11 / 30 / 82$ & 16.4 & 61.5 & 74.3 \\
\hline $12 / 01 / 82$ & $-12 / 31 / 82$ & 13.9 & 57.0 & 81.1 \\
\hline $1 / 01 / 83$ & $-1 / 31 / 83$ & 9.5 & 49.1 & 81.1 \\
\hline $2 / 01 / 83$ & $-\quad 2 / 28 / 83$ & 11.3 & 52.4 & 77.3 \\
\hline $3 / 01 / 83$ & $-\quad 3 / 31 / 83$ & 14.2 & 57.6 & 73.5 \\
\hline $4 / 01 / 83$ & $-\quad 4 / 30 / 83$ & 17.5 & 63.5 & 73.4 \\
\hline $5 / 01 / 83$ & $-5 / 31 / 83$ & 23.0 & 73.4 & 77.1 \\
\hline $6 / 01 / 83$ & $-6 / 30 / 83$ & 25.6 & 78.0 & 81.3 \\
\hline $7 / 01 / 83$ & $-7 / 31 / 83$ & 28.2 & 92.8 & 78.1 \\
\hline $8 / 01 / 83$ & $-\quad 8 / 31 / 83$ & 27.8 & 82.1 & 81.4 \\
\hline $9 / 01 / 83$ & $-\quad 9 / 30 / 83$ & 24.2 & 75.6 & 77.9 \\
\hline $10 / 01 / 83$ & $-10 / 31 / 83$ & 21.1 & 69.9 & 73.3 \\
\hline $11 / 01 / 83$ & $-11 / 30 / 83$ & 16.7 & 62.1 & 75.8 \\
\hline $12 / 01 / 83$ & $-12 / 31 / 83$ & 9.1 & 48.3 & 73.3 \\
\hline $1 / 01 / 84$ & $-1 / 31 / 84$ & 8.9 & 48.1 & 74.3 \\
\hline $2 / 01 / 84$ & $-2 / 29 / 84$ & 13.3 & 55.9 & 68.1 \\
\hline $3 / 01 / 84$ & $-3 / 31 / 84$ & 16.9 & 62.4 & 72.5 \\
\hline $4 / 01 / 84$ & $-4 / 30 / 84$ & 21.1 & 69.9 & 66.9 \\
\hline $5 / 01 / 84$ & $-5 / 31 / 84$ & 23.9 & 75.0 & 72.3 \\
\hline $6 / 01 / 84$ & $-6 / 18 / 84$ & 26.4 & 79.5 & 79.0 \\
\hline
\end{tabular}




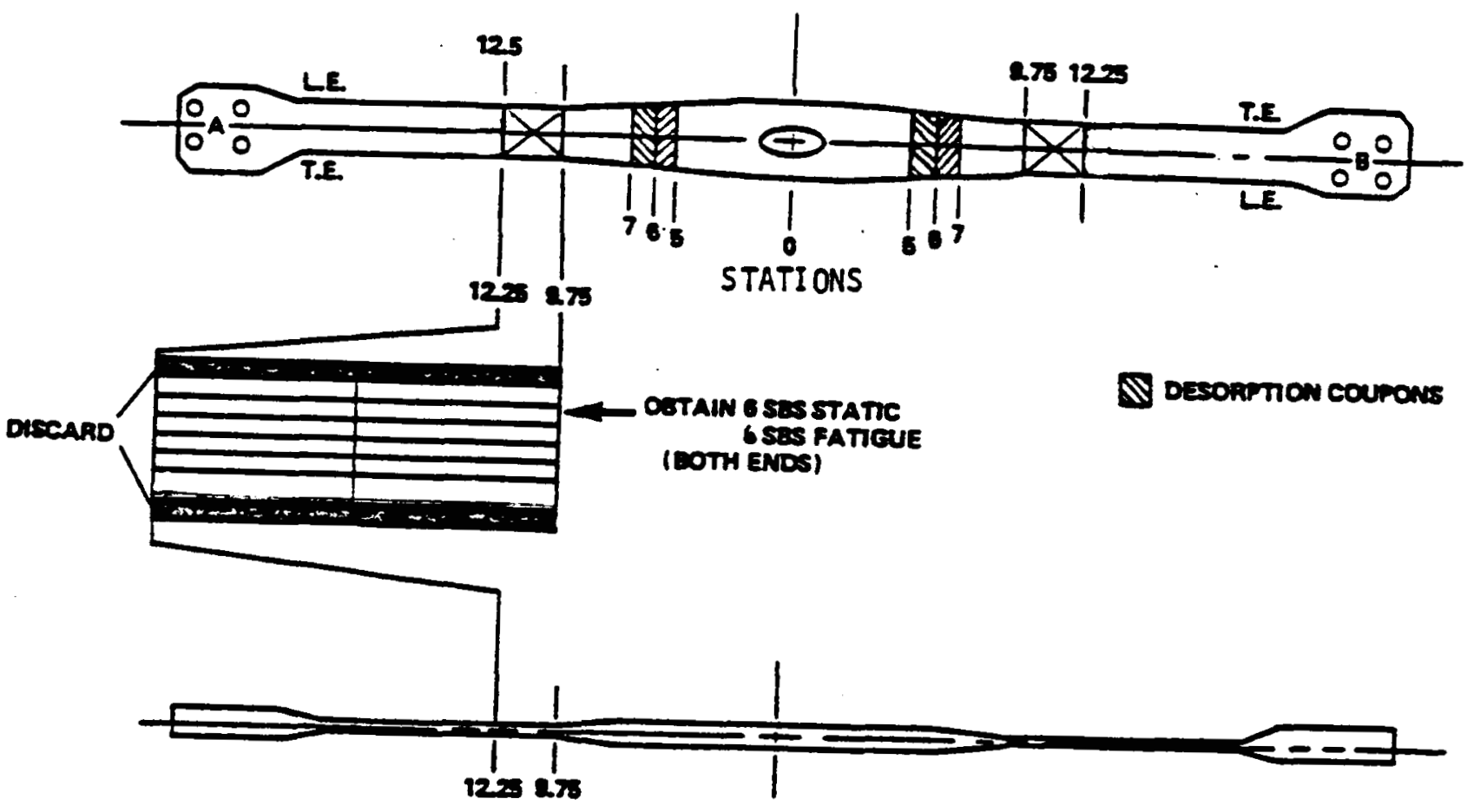

FIGURE 7. S-76 TAIL ROTOR SPAR - SKETCH OF COUPON LOCATIONS 
TABLE IX. SHORT BEAM SHEAR STRENGTH AT ROOM TEMPERATURE, S/N A-116-00178 TAIL ROTOR SPAR

\begin{tabular}{llccc}
\hline $\begin{array}{l}\text { SPECIMEN } \\
\text { NUMBER }\end{array}$ & $\begin{array}{l}\text { WIDTH } \\
\text { (in) }\end{array}$ & $\begin{array}{c}\text { THICKNESS } \\
\text { (in) }\end{array}$ & $\begin{array}{l}\text { LOAD } \\
\text { (lb) }\end{array}$ & $\begin{array}{l}\text { SBS } \\
\text { STRENGTH } \\
\text { (ksi) }\end{array}$ \\
\hline A1 & .295 & .177 & 870 & 12.5 \\
A2 & .290 & .179 & 830 & 13.4 \\
A3 & .290 & .176 & 855 & 12.6 \\
B1 & .283 & .180 & 890 & 13.1 \\
B2 & .278 & .181 & 885 & 13.2 \\
B3 & .220 & .179 & 690 & 13.1 \\
\hline & & & & \\
$\bar{X}$ & & & & 13.0 \\
\hline
\end{tabular}

TABLE X. SHORT BEAM SHEAR STRENGTH AT $170^{\circ} \mathrm{F}$, S/N A-116-00178 TAIL ROTOR SPAR

\begin{tabular}{llclc}
\hline $\begin{array}{l}\text { SPECIMEN } \\
\text { NUMBER }\end{array}$ & $\begin{array}{l}\text { WIDTH } \\
\text { (in) }\end{array}$ & $\begin{array}{c}\text { THICKNESS } \\
\text { (in) }\end{array}$ & $\begin{array}{l}\text { LOAD } \\
\text { (lb) }\end{array}$ & $\begin{array}{l}\text { SBS } \\
\text { STRENGTH } \\
\text { (ksi) }\end{array}$ \\
\hline A4 & .260 & .177 & 660 & 10.8 \\
A5 & .255 & .177 & 605 & 10.1 \\
A6 & .280 & .176 & 675 & 10.3 \\
B4 & .283 & .184 & 695 & 10.0 \\
B5 & .281 & .180 & 670 & 9.9 \\
B6 & .209 & .181 & 510 & 10.1 \\
\hline & & & & 10.2 \\
\hline
\end{tabular}


FOR ROOM TEMPERATURE TEST DATA

$\begin{array}{ll}\frac{X_{1}}{12.5} & \frac{X_{2}}{13.1} \\ 13.4 & 13.2 \\ 12.6 & 13.1\end{array}$

$$
\begin{aligned}
\mathrm{n}_{1} & =3 & \mathrm{n}_{2} & =3 \\
\overline{\mathrm{X}}_{1} & =12.8 & \overline{\mathrm{X}}_{2} & =13.1 \\
\Sigma \mathrm{X}_{1}^{2} & =0.49 & \Sigma \cdot \mathrm{X}_{2}^{2} & =0.01
\end{aligned}
$$

\begin{tabular}{|c|c|}
\hline$X_{1}$ & $X_{2}$ \\
\hline 10.8 & 10.0 \\
\hline 10.1 & 9.93 \\
\hline 10.3 & 10.1 \\
\hline
\end{tabular}$$
\bar{S}(X)=\sqrt{\frac{.49+.01}{3+3-2}}
$$$$
\overline{\mathrm{S}}(\mathrm{X})=.3536
$$$$
t=\frac{|12.8-13.1|}{.3536 \sqrt{\frac{1}{3}+\frac{1}{3}}}
$$$$
t=1.04<t .05,4=2.776
$$

FOR $170^{\circ} \mathrm{F}$ DATA

$$
\begin{aligned}
\mathrm{n}_{1} & =3 & \mathrm{n}_{2} & =3 \\
\overline{\mathrm{X}}_{1} & =10.4 & \overline{\mathrm{X}}_{2} & =10.0 \\
\Sigma^{\prime} \mathrm{X}_{1}^{2} & =0.26 & \Sigma^{\prime} \mathrm{X}_{2}^{2} & =0.0146
\end{aligned}
$$$$
\bar{S}(X)=\sqrt{\frac{.26+.0146}{3+3-2}}
$$$$
\overline{\mathrm{S}}(\mathrm{X})=.262
$$$$
t=\frac{|10.4-10.0|}{.262 \sqrt{\frac{1}{3}+\frac{1}{3}}}
$$

$t=1.82<t .05,4=2.776$

FIGURE 8. T-TEST CALCULATIONS TO DETERMINE IF TEST RESULTS FROM A AND B ENDS OF TAIL ROTOR SPAR S/N A-116-00178 ARE FROM THE SAME POPULATION 
TABLE XI COMPILATION OF SPAR COUPON STATIC TESTING WITH COMPARISON TO PANEL TEST RESULTS

ROOM TEMPERATURE DATA

\begin{tabular}{lcccc}
\hline $\begin{array}{l}\text { SPAR } \\
\text { S/N }\end{array}$ & $\begin{array}{c}\text { EXPOSURE } \\
\text { TIME } \\
\text { (MONTHS) }\end{array}$ & $\begin{array}{l}\text { FLIGHT } \\
\text { HOURS }\end{array}$ & $\begin{array}{c}\text { COUPON } \\
\text { SBS STRENGTH } \\
\text { (KSI) }\end{array}$ & $\begin{array}{l}\text { PANEL } \\
\text { STRENGTH } \\
\text { (KSI) }\end{array}$ \\
\hline 00150 & 37 & 2385 & 12.2 & $13.3^{1 /}$ \\
00283 & 37 & 1884 & 12.3 & $13.3 \underline{1 /}$ \\
00178 & 55 & 3753 & 13.0 & $13.0^{2} /$ \\
\hline
\end{tabular}

NOTES: 1/ Panel exposure time (months) $=34.5$

2/ Panel exposure time (months) $=48$ 
TABLE XII COMPILATION OF SPAR COUPON STATIC TESTING WITH COMPARISON TO PANEL TEST RESULTS $170^{\circ} \mathrm{F}$ DATA

\begin{tabular}{lcccc}
\hline $\begin{array}{l}\text { SPAR } \\
\text { S/N }\end{array}$ & $\begin{array}{c}\text { EXPOSURE } \\
\text { TIME } \\
\text { (MONTHS) }\end{array}$ & $\begin{array}{l}\text { FLIGHT } \\
\text { HOURS }\end{array}$ & $\begin{array}{c}\text { COUPON } \\
\text { SBS } \begin{array}{l}\text { STRENGTH } \\
\text { (KSI) }\end{array}\end{array}$ & $\begin{array}{c}\text { PANEL } \\
\text { SBS STRENGTH } \\
\text { (KSI) }\end{array}$ \\
\hline 00150 & 37 & 2385 & 8.5 & $7.8^{-1 /}$ \\
00283 & 37 & 1884 & 9.5 & $7.8^{\frac{1}{}}$ \\
00178 & 55 & 3753 & 10.2 & $9.8^{2 /}$ \\
\hline
\end{tabular}

NOTES: 1/ Panel exposure time (months) $=34.5$

2/ Panel exposure time (months) $=48$ 


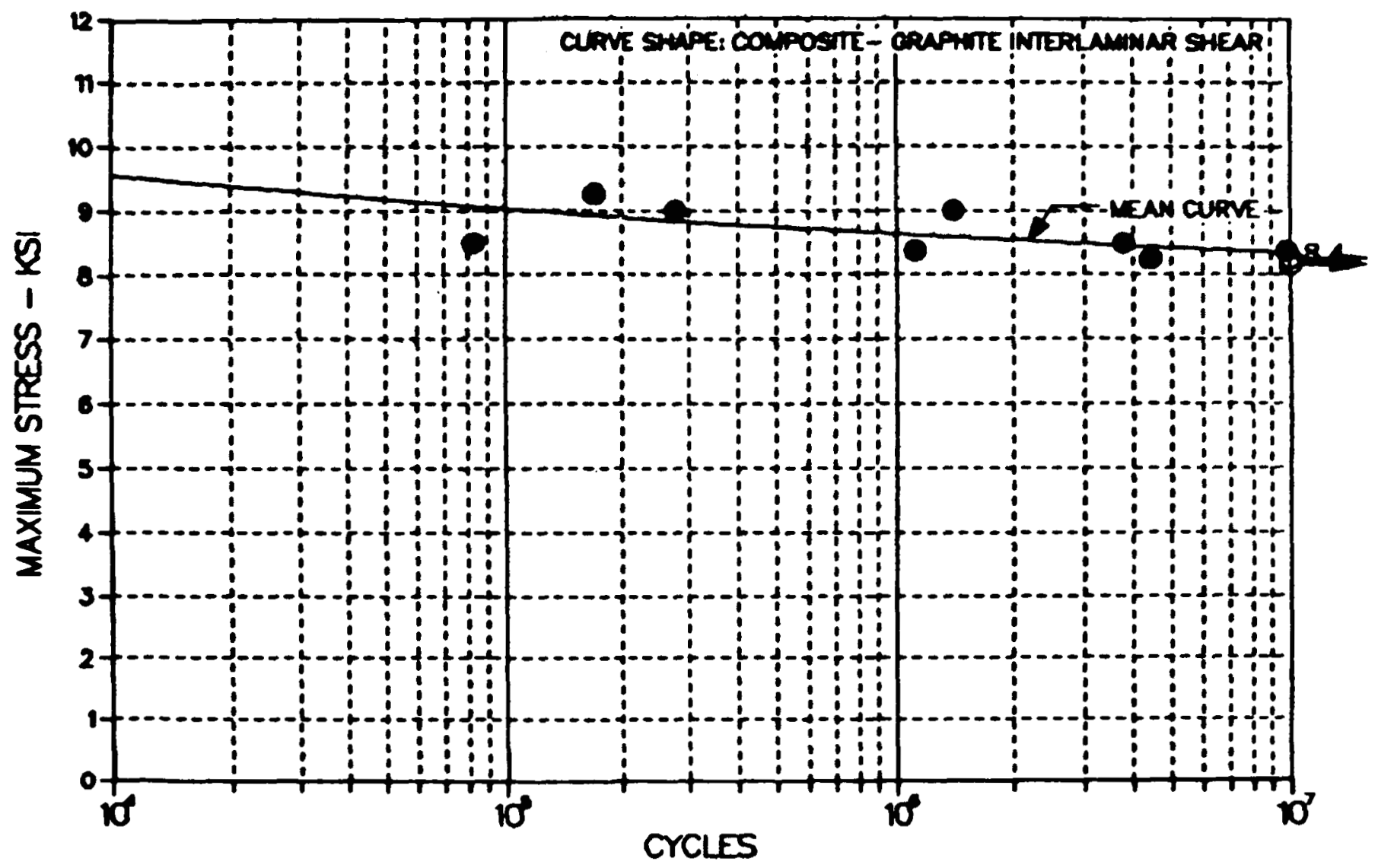

$\beta=.138 \quad \gamma=.138 \quad E_{-}=7.59 \quad \sqrt{x}=3.62 x$

FIGURE 9. SPAR S/N A-116-00178

INTERLAMINAR SHEAR FATIGUE

COUPON TESTING - MAXIMUM

STRESS VERSUS CYCLES TO

FRACTURE 
TABLE XIII. COMPILATION OF SPAR COUPON FATIGUE TESTING ROOM TEMPERATURE DATA

\begin{tabular}{lclc}
\hline $\begin{array}{l}\text { Spar } \\
\text { S/N }\end{array}$ & $\begin{array}{c}\text { Exposure } \\
\text { Time } \\
\text { (Months) }\end{array}$ & $\begin{array}{l}\text { Flight } \\
\text { Hours }\end{array}$ & $\begin{array}{c}\text { Max. Stress (KSI) } \\
\text { at } 10^{7} \text { Cycles }\end{array}$ \\
\hline 00150 & 38 & 2,385 & 7.4 \\
00283 & 38 & 1,884 & 7.4 \\
00178 & 55 & 3,753 & 8.4 \\
\hline
\end{tabular}




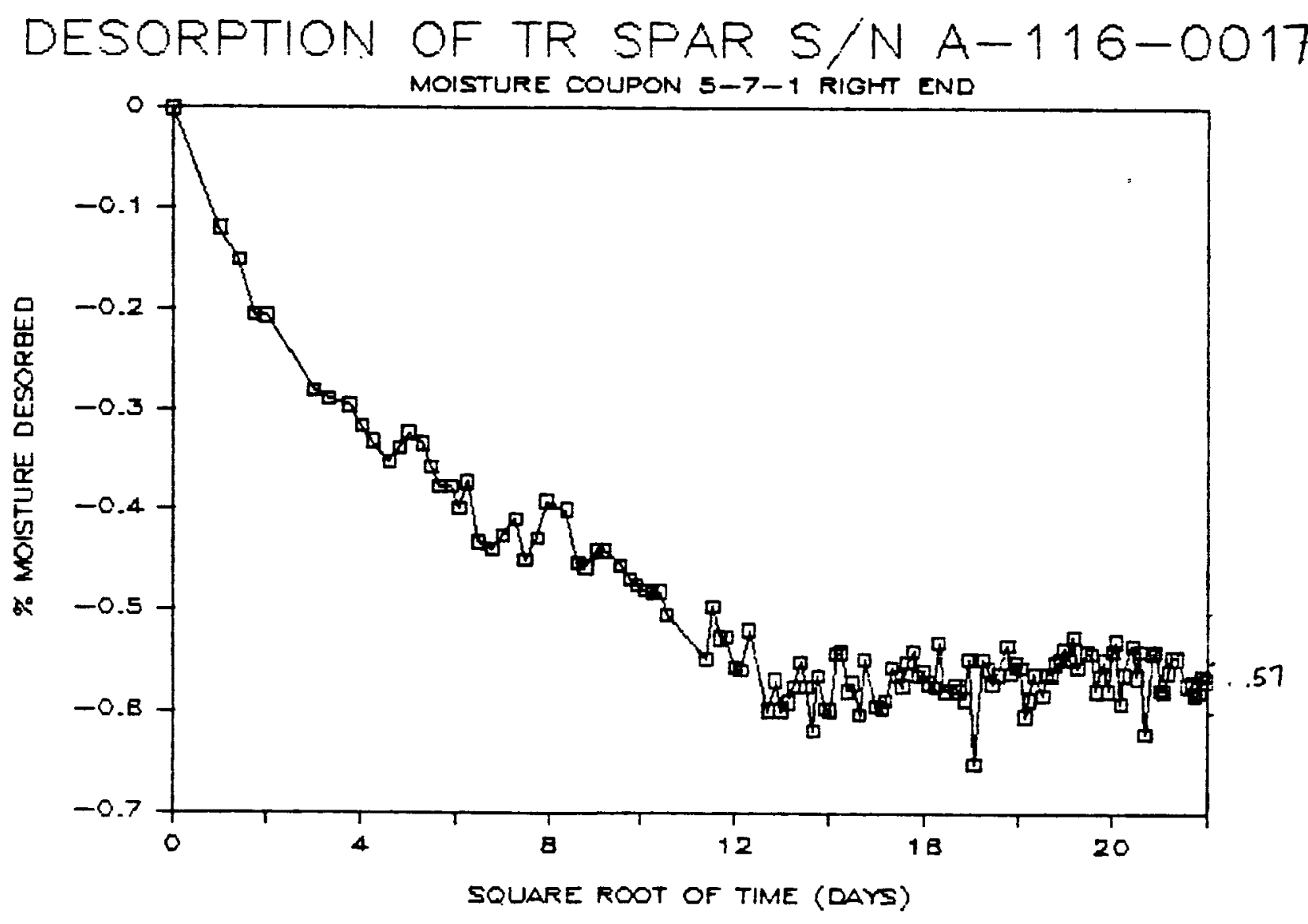

FIGURE 10. MOISTURE DESORPTION OF TAIL ROTOR SPAR S/N A-116-00178, COUPON 5-7-1, RIGHT 
TABLE XIV MOISTURE DESORPTION OF TAIL ROTOR SPAR

S/N A-116-00178, BUTTLINES 5-7, A END

\begin{tabular}{|c|c|c|c|c|c|c|c|}
\hline $\begin{array}{l}\text { DATE OF } \\
\text { WEIGHING }\end{array}$ & DAYS & $\begin{array}{c}\text { SQ RT } \\
\text { OF TIME }\end{array}$ & $\begin{array}{l}\% \text { MOIST } \\
\text { DESORBED } \\
\text { COUPON } \\
5-7-1\end{array}$ & $\begin{array}{c}\% \text { MOIST } \\
\text { DESORBED } \\
\text { COUPON } \\
5-7-2\end{array}$ & $\begin{array}{c}\% \text { MOIST } \\
\text { DESORBED } \\
\text { COUPON } \\
5-7-3\end{array}$ & $\begin{array}{c}\% \text { MOIST } \\
\text { DESORBED } \\
\text { COUPON } \\
5-7-4\end{array}$ & $\begin{array}{l}\text { AVERAGE } \\
\% \text { MOIST } \\
\text { DESORBED }\end{array}$ \\
\hline $8 / 27 / 84$ & 0 & 0.00 & .00 & .00 & .00 & .00 & .00 \\
\hline $8 / 28 / 84$ & 1 & 1.00 & -0.12 & -0.13 & -0.13 & -0.15 & -0.13 \\
\hline $8 / 29 / 84$ & 2 & 1.41 & -0.15 & -0.17 & -0.16 & -0.18 & -0.16 \\
\hline $8 / 30 / 84$ & 3 & 1.73 & -0.20 & -0.19 & -0.20 & -0.21 & -0.20 \\
\hline $8 / 31 / 84$ & 4 & 2.00 & -0.21 & -0.22 & -0.21 & -0.22 & -0.21 \\
\hline $9 / 05 / 84$ & 9 & 3.00 & -0.28 & -0.30 & -0.28 & -0.31 & -0.29 \\
\hline $9 / 07 / 84$ & 11 & 3.32 & -0.29 & -0.28 & -0.28 & -0.34 & -0.30 \\
\hline $9 / 10 / 84$ & 14 & 3.74 & -0.30 & -0.30 & -0.28 & -0.36 & -0.31 \\
\hline $9 / 12 / 84$ & 16 & 4.00 & -0.32 & -0.33 & -0.29 & -0.38 & -0.33 \\
\hline $9 / 14 / 84$ & 18 & 4.24 & -0.33 & -0.33 & -0.30 & -0.37 & -0.33 \\
\hline $9 / 17 / 84$ & 21 & 4.58 & -0.35 & -0.38 & -0.36 & -0.42 & -0.38 \\
\hline $9 / 19 / 84$ & 23 & 4.80 & -0.34 & -0.33 & -0.31 & -0.38 & -0.34 \\
\hline $9 / 21 / 84$ & 25 & 5.00 & -0.32 & -0.35 & -0.31 & -0.42 & -0.35 \\
\hline $9 / 24 / 84$ & 28 & 5.29 & -0.33 & -0.34 & -0.33 & -0.41 & -0.35 \\
\hline $9 / 26 / 84$ & 30 & 5.48 & -0.36 & -0.34 & -0.32 & -0.42 & -0.36 \\
\hline $9 / 28 / 84$ & 32 & 5.66 & -0.38 & -0.40 & -0.36 & -0.46 & -0.40 \\
\hline $10 / 01 / 84$ & 35 & 5.92 & -0.38 & -0.37 & -0.39 & -0.48 & -0.40 \\
\hline $10 / 03 / 84$ & 37 & 6.08 & -0.40 & -0.41 & -0.36 & -0.44 & -0.40 \\
\hline $10 / 05 / 84$ & 39 & 6.24 & -0.37 & -0.38 & -0.36 & -0.47 & -0.40 \\
\hline $10 / 08 / 84$ & 42 & 6.48 & -0.43 & -0.41 & -0.40 & -0.51 & -0.44 \\
\hline $10 / 12 / 84$ & 46 & 6.78 & -0.44 & -0.45 & -0.41 & -0.49 & -0.45 \\
\hline $10 / 15 / 84$ & 49 & 7.00 & -0.43 & -0.43 & -0.38 & -0.53 & -0.44 \\
\hline $10 / 19 / 84$ & 53 & 7.28 & -0.41 & -0.41 & -0.38 & -0.50 & -0.43 \\
\hline $10 / 22 / 84$ & 56 & 7.48 & -0.45 & -0.45 & -0.42 & -0.52 & -0.46 \\
\hline $10 / 26 / 84$ & 60 & 7.75 & -0.43 & -0.43 & -0.41 & -0.53 & -0.45 \\
\hline $10 / 29 / 84$ & 63 & 7.94 & -0.39 & -0.39 & -0.36 & -0.49 & -0.41 \\
\hline $11 / 05 / 84$ & 70 & 8.37 & -0.40 & -0.40 & -0.38 & -0.50 & -0.42 \\
\hline $11 / 09 / 84$ & 74 & 8.60 & -0.45 & -0.45 & -0.40 & -0.54 & -0.46 \\
\hline $11 / 12 / 84$ & 77 & 8.77 & -0.46 & -0.45 & -0.39 & -0.53 & -0.46 \\
\hline $11 / 16 / 84$ & 81 & 9.00 & -0.44 & -0.44 & -0.41 & -0.52 & -0.45 \\
\hline $11 / 19 / 84$ & 84 & 9.17 & -0.44 & -0.44 & -0.44 & -0.56 & -0.47 \\
\hline $11 / 26 / 84$ & 91 & 9.54 & -0.46 & -0.46 & -0.44 & -0.56 & -0.48 \\
\hline $11 / 30 / 84$ & 95 & 9.75 & -0.47 & -0.48 & -0.43 & -0.56 & -0.49 \\
\hline $12 / 03 / 84$ & 98 & 9.90 & -0.48 & -0.50 & -0.44 & -0.60 & -0.51 \\
\hline $12 / 07 / 84$ & 102 & 10.10 & -0.48 & -0.48 & -0.45 & -0.58 & -0.50 \\
\hline $12 / 10 / 84$ & 105 & 10.25 & -0.48 & -0.50 & -0.46 & -0.57 & -0.50 \\
\hline $12 / 14 / 84$ & 109 & 10.44 & -0.48 & -0.48 & -0.45 & -0.60 & -0.50 \\
\hline $12 / 17 / 84$ & 112 & 10.58 & -0.51 & -0.51 & -0.48 & -0.60 & -0.53 \\
\hline
\end{tabular}


TABLE XIV (cont'd) MOISTURE DESORPTION OF TAIL ROTOR SPAR S/N A-116-00178, BUTTLINES 5-7, A END

\begin{tabular}{|c|c|c|c|c|c|c|c|}
\hline $\begin{array}{l}\text { DATE OF } \\
\text { WEIGHING }\end{array}$ & DAYS & $\begin{array}{c}\text { SQ RT } \\
\text { OF TIME }\end{array}$ & $\begin{array}{l}\% \text { MOIST } \\
\text { DESORBED } \\
\text { COUPON } \\
5-7-1\end{array}$ & $\begin{array}{l}\% \text { MOIST } \\
\text { DESORBED } \\
\text { COUPON } \\
5-7-2\end{array}$ & $\begin{array}{c}\% \text { MOIST } \\
\text { DESORBED } \\
\text { COUPON } \\
5-7-3\end{array}$ & $\begin{array}{l}\% \text { MOIST } \\
\text { DESORBED } \\
\text { COUPON } \\
5-7-4\end{array}$ & $\begin{array}{l}\text { AVERAGE } \\
\% \text { MOIST } \\
\text { DESORBED }\end{array}$ \\
\hline $1 / 04 / 85$ & 130 & 11.40 & -0.55 & -0.55 & -0.48 & -0.62 & -0.55 \\
\hline $1 / 07 / 85$ & 133 & 11.53 & -0.50 & -0.52 & -0.48 & -0.62 & -0.53 \\
\hline $1 / 11 / 85$ & 137 & 11.70 & -0.53 & -0.54 & -0.49 & -0.62 & -0.54 \\
\hline $1 / 14 / 85$ & 140 & 11.83 & -0.53 & -0.52 & -0.48 & -0.61 & -0.54 \\
\hline $1 / 18 / 85$ & 144 & 12.00 & -0.56 & -0.56 & -0.52 & -0.63 & -0.57 \\
\hline $1 / 21 / 85$ & 147 & 12.12 & -0.56 & -0.55 & -0.53 & -0.64 & -0.57 \\
\hline $1 / 25 / 85$ & 151 & 12.29 & -0.52 & -0.54 & -0.49 & -0.62 & -0.54 \\
\hline $2 / 04 / 85$ & 161 & 12.69 & -0.60 & -0.60 & -0.56 & -0.70 & -0.62 \\
\hline $2 / 08 / 85$ & 165 & 12.83 & -0.57 & -0.58 & -0.55 & -0.66 & -0.59 \\
\hline $2 / 11 / 85$ & 168 & 12.96 & -0.60 & -0.62 & -0.56 & -0.70 & -0.62 \\
\hline $2 / 15 / 85$ & 172 & 13.11 & -0.59 & -0.59 & -0.57 & -0.67 & -0.61 \\
\hline $2 / 18 / 85$ & 175 & 13.23 & -0.58 & -0.59 & -0.52 & -0.68 & -0.59 \\
\hline $2 / 22 / 85$ & 179 & 13.38 & -0.55 & -0.57 & -0.54 & -0.68 & -0.58 \\
\hline $2 / 25 / 85$ & 182 & 13.49 & -0.58 & -0.57 & -0.53 & -0.67 & -0.59 \\
\hline $3 / 01 / 85$ & 186 & 13.64 & -0.62 & -0.58 & -0.56 & -0.71 & -0.62 \\
\hline $3 / 04 / 85$ & 189 & 13.75 & -0.56 & -0.59 & -0.53 & -0.67 & -0.59 \\
\hline $3 / 08 / 85$ & 193 & 13.89 & -0.60 & -0.59 & -0.54 & -0.71 & -0.61 \\
\hline $3 / 11 / 85$ & 196 & 14.00 & -0.60 & -0.62 & -0.57 & -0.71 & -0.62 \\
\hline $3 / 15 / 85$ & 200 & 14.14 & -0.54 & -0.57 & -0.52 & -0.65 & -0.57 \\
\hline $3 / 18 / 85$ & 203 & 14.25 & -0.54 & -0.55 & -0.54 & -0.69 & -0.58 \\
\hline $3 / 22 / 85$ & 207 & 14.39 & -0.58 & -0.59 & -0.54 & -0.70 & -0.60 \\
\hline $3 / 25 / 85$ & 210 & 14.49 & -0.57 & -0.59 & -0.57 & -0.69 & -0.60 \\
\hline $3 / 29 / 85$ & 214 & 14.63 & -0.60 & -0.61 & -0.56 & -0.70 & -0.62 \\
\hline $4 / 01 / 85$ & 217 & 14.73 & -0.55 & -0.60 & -0.54 & -0.72 & -0.60 \\
\hline $4 / 08 / 85$ & 224 & 14.97 & -0.59 & -0.62 & -0.59 & -0.75 & -0.64 \\
\hline $4 / 12 / 85$ & 228 & 15.10 & -0.60 & -0.61 & -0.56 & -0.72 & -0.62 \\
\hline $4 / 15 / 85$ & 231 & 15.20 & -0.59 & -0.60 & -0.52 & -0.70 & -0.60 \\
\hline $4 / 19 / 85$ & 235 & 15.33 & -0.56 & -0.60 & -0.51 & -0.67 & -0.58 \\
\hline $4 / 22 / 85$ & 238 & 15.43 & -0.56 & -0.59 & -0.51 & -0.66 & -0.58 \\
\hline $4 / 26 / 85$ & 242 & 15.56 & -0.57 & -0.62 & -0.54 & -0.69 & -0.61 \\
\hline $4 / 29 / 85$ & 245 & 15.65 & -0.55 & -0.58 & -0.52 & -0.68 & -0.58 \\
\hline $5 / 03 / 85$ & 249 & 15.78 & -0.54 & -0.56 & -0.50 & -0.67 & -0.57 \\
\hline $5 / 06 / 85$ & 252 & 15.87 & -0.57 & -0.58 & -0.54 & -0.70 & -0.60 \\
\hline $5 / 10 / 85$ & 256 & 16.00 & -0.56 & -0.59 & -0.52 & -0.69 & -0.59 \\
\hline $5 / 13 / 85$ & 259 & 16.09 & -0.57 & -0.58 & -0.51 & -0.71 & -0.59 \\
\hline $5 / 17 / 85$ & 263 & 16.22 & -0.58 & -0.60 & -0.52 & -0.66 & -0.59 \\
\hline $5 / 20 / 85$ & 266 & 16.31 & -0.53 & -0.55 & -0.48 & -0.65 & -0.55 \\
\hline $5 / 24 / 85$ & 270 & 16.43 & -0.58 & -0.62 & -0.55 & -0.72 & -0.62 \\
\hline
\end{tabular}


TABLE XIV (cont'd) MOISTURE DESORPTION OF TAIL ROTOR SPAR

S/N A-116-00178, BUTTLINES 5-7, A END

\begin{tabular}{|c|c|c|c|c|c|c|c|}
\hline $\begin{array}{l}\text { DATE OF } \\
\text { WEIGHING }\end{array}$ & DAYS & $\begin{array}{c}\text { SQ RT } \\
\text { OF TIME }\end{array}$ & $\begin{array}{c}\% \text { MOIST } \\
\text { DESORBED } \\
\text { COUPON } \\
5-7-1\end{array}$ & $\begin{array}{l}\% \text { MOIST } \\
\text { DESORBED } \\
\text { COUPON } \\
5-7-2\end{array}$ & $\begin{array}{c}\% \text { MOIST } \\
\text { DESORBED } \\
\text { COUPON } \\
5-7-3\end{array}$ & $\begin{array}{c}\% \text { MOIST } \\
\text { DESORBED } \\
\text { COUPON } \\
5-7-4\end{array}$ & $\begin{array}{l}\text { AVERAGE } \\
\% \text { MOIST } \\
\text { DESORBED }\end{array}$ \\
\hline $5 / 31 / 85$ & 277 & 16.64 & -0.57 & -0.60 & -0.51 & -0.68 & -0.59 \\
\hline $6 / 03 / 85$ & 280 & 16.73 & -0.58 & -0.56 & -0.51 & -0.66 & -0.58 \\
\hline $6 / 07 / 85$ & 284 & 16.85 & -0.59 & -0.63 & -0.54 & -0.70 & -0.61 \\
\hline $6 / 10 / 85$ & 287 & 16.94 & -0.55 & -0.56 & -0.49 & -0.65 & -0.56 \\
\hline $6 / 14 / 85$ & 291 & 17.06 & -0.65 & -0.67 & -0.62 & -0.77 & -0.68 \\
\hline $6 / 17 / 85$ & 297 & 17.23 & -0.55 & -0.57 & -0.52 & -0.66 & -0.57 \\
\hline $6 / 21 / 85$ & 301 & 17.35 & -0.56 & -0.57 & -0.53 & -0.67 & -0.58 \\
\hline $6 / 24 / 85$ & 304 & 17.44 & -0.57 & -0.59 & -0.52 & -0.66 & -0.59 \\
\hline $6 / 28 / 85$ & 308 & 17.55 & -0.56 & -0.57 & -0.52 & -0.67 & -0.58 \\
\hline $7 / 05 / 85$ & 315 & 17.75 & -0.53 & -0.55 & -0.48 & -0.64 & -0.55 \\
\hline $7 / 08 / 85$ & 318 & 17.83 & -0.56 & -0.57 & -0.52 & -0.66 & -0.58 \\
\hline $7 / 12 / 85$ & 322 & 17.94 & -0.55 & -0.57 & -0.54 & -0.67 & -0.58 \\
\hline $7 / 15 / 85$ & 325 & 18.03 & -0.56 & -0.58 & -0.53 & -0.67 & -0.58 \\
\hline $7 / 19 / 85$ & 329 & 18.14 & -0.60 & -0.61 & -0.56 & -0.72 & -0.62 \\
\hline $7 / 22 / 85$ & 332 & 18.22 & -0.59 & -0.60 & -0.55 & -0.70 & -0.61 \\
\hline $7 / 26 / 85$ & 336 & 18.33 & -0.56 & -0.57 & -0.52 & -0.66 & -0.58 \\
\hline $8 / 02 / 85$ & 343 & 18.52 & -0.58 & -0.58 & -0.55 & -0.68 & -0.60 \\
\hline $8 / 05 / 85$ & 346 & 18.60 & -0.56 & -0.56 & -0.52 & -0.68 & -0.58 \\
\hline $8 / 09 / 85$ & 350 & 18.71 & -0.56 & -0.59 & -0.53 & -0.70 & -0.60 \\
\hline $8 / 12 / 85$ & 353 & 18.79 & -0.55 & -0.58 & -0.50 & -0.66 & -0.57 \\
\hline $8 / 16 / 85$ & 357 & 18.89 & -0.55 & -0.56 & -0.52 & -0.68 & -0.58 \\
\hline $8 / 19 / 85$ & 360 & 18.97 & -0.54 & -0.58 & -0.54 & -0.69 & -0.59 \\
\hline $8 / 23 / 85$ & 364 & 19.08 & -0.55 & -0.59 & -0.52 & -0.69 & -0.59 \\
\hline $8 / 26 / 85$ & 367 & 19.16 & -0.53 & -0.55 & -0.50 & -0.66 & -0.56 \\
\hline $8 / 30 / 85$ & 371 & 19.26 & -0.56 & -0.58 & -0.51 & -0.68 & -0.58 \\
\hline $9 / 06 / 85$ & 378 & 19.44 & -0.54 & -0.55 & -0.50 & -0.65 & -0.56 \\
\hline $9 / 09 / 85$ & 382 & 19.54 & -0.54 & -0.57 & -0.49 & -0.66 & -0.57 \\
\hline $9 / 13 / 85$ & 386 & 19.65 & -0.58 & -0.58 & -0.50 & -0.68 & -0.59 \\
\hline $9 / 16 / 85$ & 389 & 19.72 & -0.57 & -0.56 & -0.49 & -0.68 & -0.57 \\
\hline $9 / 20 / 85$ & 393 & 19.82 & -0.55 & -0.56 & -0.49 & -0.65 & -0.56 \\
\hline $9 / 23 / 85$ & 396 & 19.90 & -0.58 & -0.57 & -0.44 & -0.68 & -0.57 \\
\hline $9 / 27 / 85$ & 400 & 20.00 & -0.54 & -0.59 & -0.52 & -0.68 & -0.58 \\
\hline $9 / 30 / 85$ & 403 & 20.07 & -0.53 & -0.56 & -0.48 & -0.64 & -0.55 \\
\hline $10 / 04 / 85$ & 407 & 20.17 & -0.59 & -0.62 & -0.56 & -0.72 & -0.62 \\
\hline $10 / 07 / 85$ & 410 & 20.25 & -0.56 & -0.37 & -0.49 & -0.67 & -0.57 \\
\hline $10 / 14 / 85$ & 417 & 20.42 & -0.54 & -0.54 & -0.48 & -0.64 & -0.55 \\
\hline
\end{tabular}


TABLE XIV (cont'd) MOISTURE DESORPTION OF TAIL ROTOR SPAR S/N A-116-00178, BUTTLINES 5-7, A END

\begin{tabular}{|c|c|c|c|c|c|c|c|}
\hline $\begin{array}{l}\text { DATE OF } \\
\text { WEIGHING }\end{array}$ & DAYS & $\begin{array}{c}\text { SQ RT } \\
\text { OF TIME }\end{array}$ & $\begin{array}{c}\% \text { MOIST } \\
\text { DESORBED } \\
\text { COUPON } \\
5-7-1\end{array}$ & $\begin{array}{c}\% \text { MOIST } \\
\text { DESORBED } \\
\text { COUPON } \\
5-7-2\end{array}$ & $\begin{array}{c}\% \text { MOIST } \\
\text { DESORBED } \\
\text { COUPON } \\
5-7-3\end{array}$ & $\begin{array}{l}\% \text { MOIST } \\
\text { DESORBED } \\
\text { COUPON } \\
5-7-4\end{array}$ & $\begin{array}{l}\text { AVERAGE } \\
\% \text { MOIST } \\
\text { DESORBED }\end{array}$ \\
\hline $10 / 18 / 85$ & 421 & 20.52 & -0.57 & -0.56 & -0.52 & -0.67 & -0.58 \\
\hline $10 / 21 / 85$ & 424 & 20.59 & -0.54 & -0.55 & -0.50 & -0.64 & -0.56 \\
\hline $10 / 25 / 85$ & 428 & 20.69 & -0.62 & -0.62 & -0.57 & -0.73 & -0.64 \\
\hline $11 / 01 / 85$ & 435 & 20.86 & -0.54 & -0.55 & -0.50 & -0.66 & -0.56 \\
\hline $11 / 04 / 85$ & 438 & 20.93 & -0.54 & -0.55 & -0.51 & -0.66 & -0.56 \\
\hline $11 / 08 / 85$ & 442 & 21.02 & -0.58 & -0.57 & -0.53 & -0.67 & -0.59 \\
\hline $11 / 11 / 85$ & 445 & 21.10 & -0.58 & -0.57 & -0.54 & -0.69 & -0.59 \\
\hline $11 / 15 / 85$ & 449 & 21.19 & -0.56 & -0.57 & -0.50 & -0.68 & -0.58 \\
\hline $11 / 18 / 85$ & 452 & 21.26 & -0.55 & -0.56 & -0.50 & -0.68 & -0.57 \\
\hline $11 / 22 / 85$ & 456 & 21.35 & -0.55 & -0.59 & -0.49 & -0.67 & -0.58 \\
\hline $12 / 02 / 85$ & 466 & 21.59 & -0.57 & -0.53 & -0.53 & -0.67 & -0.58 \\
\hline $12 / 06 / 85$ & 470 & 21.68 & -0.57 & -0.57 & -0.51 & -0.70 & -0.59 \\
\hline $12 / 09 / 85$ & 473 & 21.75 & -0.58 & -0.61 & -0.54 & -0.70 & -0.61 \\
\hline $12 / 13 / 85$ & 477 & 21.84 & -0.58 & -0.58 & -0.54 & -0.68 & -0.59 \\
\hline $12 / 16 / 85$ & 480 & 21.91 & -0.56 & -0.57 & -0.52 & -0.67 & -0.58 \\
\hline $12 / 20 / 85$ & 484 & 22.00 & -0.57 & -0.58 & -0.53 & -0.66 & -0.59 \\
\hline
\end{tabular}


TABLE XV MOISTURE DESORPTION OF TAIL ROTOR SPAR S/N A-116-00178, BUTTLINES 5-7, B END

\begin{tabular}{|c|c|c|c|c|c|c|c|}
\hline $\begin{array}{l}\text { DATE OF } \\
\text { WEIGHING }\end{array}$ & DAYS & $\begin{array}{c}\text { SQ RT } \\
\text { OF TIME }\end{array}$ & $\begin{array}{c}\% \text { MOIST } \\
\text { DESORBED } \\
\text { COUPON } \\
5-7-1\end{array}$ & $\begin{array}{c}\% \text { MOIST } \\
\text { DESORBED } \\
\text { COUPON } \\
5-7-2\end{array}$ & $\begin{array}{l}\% \text { MOIST } \\
\text { DESORBED } \\
\text { COUPON } \\
5-7-3\end{array}$ & $\begin{array}{l}\% \text { MOIST } \\
\text { DESORBED } \\
\text { COUPON } \\
5-7-4\end{array}$ & $\begin{array}{l}\text { AVERAGE } \\
\% \text { MOIST } \\
\text { DESORBED }\end{array}$ \\
\hline $8 / 27 / 84$ & 0 & 0.00 & 0.00 & 0.00 & 0.00 & .00 & .00 \\
\hline $8 / 28 / 84$ & 1 & 1.00 & -0.12 & -0.12 & -0.13 & -0.17 & -0.14 \\
\hline $8 / 29 / 84$ & 2 & 1.41 & -0.17 & -0.18 & -0.17 & -0.22 & -0.19 \\
\hline $8 / 30 / 84$ & 3 & 1.73 & -0.19 & -0.23 & -0.18 & -0.26 & -0.22 \\
\hline $8 / 31 / 84$ & 4 & 2.00 & -0.19 & -0.20 & -0.22 & -0.27 & -0.22 \\
\hline $9 / 05 / 84$ & 9 & 3.00 & -0.27 & -0.31 & -0.29 & -0.36 & -0.31 \\
\hline $9 / 07 / 84$ & 11 & 3.32 & -0.29 & -0.32 & -0.32 & -0.36 & -0.32 \\
\hline $9 / 10 / 84$ & 14 & 3.74 & -0.30 & -0.31 & -0.30 & -0.40 & -0.33 \\
\hline $9 / 12 / 84$ & 16 & 4.00 & -0.32 & -0.35 & -0.34 & -0.40 & -0.35 \\
\hline $9 / 14 / 84$ & 18 & 4.24 & -0.33 & -0.32 & -0.33 & -0.43 & -0.35 \\
\hline $9 / 17 / 84$ & 21 & 4.58 & -0.38 & -0.40 & -0.39 & -0.48 & -0.41 \\
\hline $9 / 19 / 84$ & 23 & 4.80 & -0.33 & -0.35 & -0.34 & -0.44 & -0.37 \\
\hline $9 / 21 / 84$ & 25 & 5.00 & -0.35 & -0.35 & -0.34 & -0.44 & -0.37 \\
\hline $9 / 24 / 84$ & 29 & 5.29 & -0.35 & -0.37 & -0.35 & -0.45 & -0.38 \\
\hline $9 / 26 / 84$ & 30 & 5.48 & -0.36 & -0.41 & -0.35 & -0.47 & -0.40 \\
\hline $9 / 28 / 84$ & 32 & 5.66 & -0.39 & -0.42 & -0.42 & -0.47 & -0.43 \\
\hline $10 / 01 / 84$ & 35 & 5.92 & -0.42 & -0.42 & -0.37 & -0.51 & -0.43 \\
\hline $10 / 03 / 84$ & 37 & 6.08 & -0.39 & -0.43 & -0.38 & -0.50 & -0.43 \\
\hline $10 / 05 / 84$ & 39 & 6.24 & -0.39 & -0.41 & -0.40 & -0.50 & -0.43 \\
\hline $10 / 08 / 84$ & 42 & 6.48 & -0.41 & -0.46 & -0.41 & -0.55 & -0.46 \\
\hline $10 / 12 / 84$ & 46 & 6.78 & -0.46 & -0.46 & -0.42 & -0.53 & -0.47 \\
\hline $10 / 15 / 84$ & 49 & 7.00 & -0.44 & -0.45 & -0.43 & -0.55 & -0.46 \\
\hline $10 / 19 / 84$ & 53 & 7.28 & -0.43 & -0.45 & -0.41 & -0.56 & -0.46 \\
\hline $10 / 22 / 84$ & 56 & 7.48 & -0.46 & -0.49 & -0.46 & -0.59 & -0.50 \\
\hline $10 / 26 / 84$ & 60 & 7.75 & -0.44 & -0.44 & -0.44 & -0.54 & -0.46 \\
\hline $10 / 29 / 84$ & 63 & 7.94 & -0.41 & -0.42 & -0.39 & -0.54 & -0.44 \\
\hline $11 / 05 / 84$ & 70 & 8.37 & -0.41 & -0.43 & -0.46 & -0.54 & -0.46 \\
\hline $11 / 09 / 84$ & 74 & 8.60 & -0.45 & -0.50 & -0.45 & -0.57 & -0.49 \\
\hline $11 / 12 / 84$ & 77 & 8.77 & -0.43 & -0.46 & -0.45 & -0.57 & -0.48 \\
\hline $11 / 16 / 84$ & 81 & 9.00 & -0.46 & -0.47 & -0.42 & -0.57 & -0.48 \\
\hline $11 / 19 / 84$ & 84 & 9.17 & -0.48 & -0.49 & -0.42 & -0.59 & -0.50 \\
\hline $11 / 26 / 84$ & 91 & 9.54 & -0.47 & -0.51 & -0.48 & -0.61 & -0.52 \\
\hline $11 / 30 / 84$ & 95 & 9.75 & -0.49 & -0.50 & -0.38 & -0.61 & -0.52 \\
\hline $12 / 03 / 84$ & 98 & 9.90 & -0.51 & -0.53 & -0.49 & -0.65 & -0.54 \\
\hline $12 / 07 / 84$ & 102 & 10.10 & -0.50 & -0.52 & -0.48 & -0.61 & -0.53 \\
\hline $12 / 10 / 84$ & 105 & 10.25 & -0.50 & -0.53 & -0.48 & -0.65 & -0.54 \\
\hline $12 / 14 / 84$ & 109 & 10.44 & -0.49 & -0.50 & -0.48 & -0.64 & -0.53 \\
\hline $12 / 17 / 84$ & 112 & 10.58 & -0.50 & -0.51 & -0.52 & -0.68 & -0.55 \\
\hline
\end{tabular}


TABLE XV (cont'd) MOISTURE DESORPTION OF TAIL ROTOR SPAR S/N A-116-00178, BUTTLINES 5-7, B END

\begin{tabular}{|c|c|c|c|c|c|c|c|}
\hline $\begin{array}{l}\text { DATE OF } \\
\text { WEIGHING }\end{array}$ & DAYS & $\begin{array}{c}\text { SQ RT } \\
\text { OF TIME }\end{array}$ & $\begin{array}{l}\% \text { MOTST } \\
\text { DESORBED } \\
\text { COUPON } \\
5-7-1\end{array}$ & $\begin{array}{l}\% \text { MOIST } \\
\text { DESORBED } \\
\text { COUPON } \\
5-7-2\end{array}$ & $\begin{array}{l}\% \text { MOIST } \\
\text { DESORBED } \\
\text { COUPON } \\
5-7-3\end{array}$ & $\begin{array}{l}\% \text { MOIST } \\
\text { DESORBED } \\
\text { COUPON } \\
5-7-4\end{array}$ & $\begin{array}{l}\text { AVERAGE } \\
\% \text { MOIST } \\
\text { DESORBED }\end{array}$ \\
\hline $1 / 04 / 85$ & 130 & 11.40 & -0.55 & -0.54 & -0.54 & -0.71 & -0.59 \\
\hline $1 / 07 / 85$ & 133 & 11.53 & -0.53 & -0.54 & -0.51 & -0.65 & -0.56 \\
\hline $1 / 11 / 85$ & 137 & 11.70 & -0.54 & -0.55 & -0.53 & -0.69 & -0.58 \\
\hline $1 / 14 / 85$ & 140 & 11.83 & -0.55 & -0.56 & -0.51 & -0.67 & -0.57 \\
\hline $1 / 18 / 85$ & 144 & 12.00 & -0.55 & -0.59 & -0.53 & -0.68 & -0.59 \\
\hline $1 / 21 / 85$ & 147 & 12.12 & -0.57 & -0.60 & -0.55 & -0.74 & -0.62 \\
\hline $1 / 25 / 85$ & 151 & 12.29 & -0.52 & -0.55 & -0.50 & -0.68 & -0.56 \\
\hline $2 / 04 / 85$ & 161 & 12.69 & -0.58 & -0.61 & -0.57 & -0.73 & -0.62 \\
\hline $2 / 08 / 85$ & 165 & 12.85 & -0.58 & -0.56 & -0.53 & -0.73 & -0.60 \\
\hline $2 / 11 / 85$ & 168 & 12.96 & -0.58 & -0.59 & -0.56 & -0.73 & -0.62 \\
\hline $2 / 15 / 85$ & 172 & 13.11 & -0.58 & -0.59 & -0.58 & -0.73 & -0.62 \\
\hline $2 / 18 / 85$ & 175 & 13.23 & -0.57 & -0.59 & -0.59 & -0.72 & -0.62 \\
\hline $2 / 22 / 85$ & 179 & 13.38 & -0.58 & -0.60 & -0.59 & -0.75 & -0.63 \\
\hline $2 / 25 / 85$ & 182 & 13.49 & -0.57 & -0.58 & -0.57 & -0.74 & -0.62 \\
\hline $3 / 01 / 85$ & 186 & 13.64 & -0.60 & -0.63 & -0.61 & -0.78 & -0.66 \\
\hline $3 / 04 / 85$ & 189 & 13.75 & -0.59 & -0.61 & -0.58 & -0.74 & -0.63 \\
\hline $3 / 08 / 85$ & 193 & 13.89 & -0.59 & -0.59 & -0.58 & -0.75 & -0.62 \\
\hline $3 / 11 / 85$ & 196 & 14.00 & -0.57 & -0.64 & -0.55 & -0.72 & -0.62 \\
\hline $3 / 15 / 85$ & 200 & 14.14 & -0.57 & -0.58 & -0.54 & -0.72 & -0.60 \\
\hline $3 / 18 / 85$ & 203 & 14.25 & -0.56 & -0.64 & -0.54 & -0.76 & -0.62 \\
\hline $3 / 22 / 85$ & 207 & 14.39 & -0.58 & -0.60 & -0.61 & -0.77 & -0.64 \\
\hline $3 / 25 / 85$ & 210 & 14.49 & -0.58 & -0.64 & -0.59 & -0.78 & -0.65 \\
\hline $3 / 29 / 85$ & 214 & 14.63 & -0.60 & -0.62 & -0.58 & -0.74 & -0.64 \\
\hline $4 / 01 / 85$ & 217 & 14.73 & -0.61 & -0.62 & -0.56 & -0.74 & -0.63 \\
\hline $4 / 08 / 85$ & 224 & 14.97 & -0.63 & -0.61 & -0.65 & -0.81 & -0.67 \\
\hline $4 / 12 / 85$ & 228 & 15.10 & -0.60 & -0.61 & -0.57 & -0.75 & -0.63 \\
\hline $4 / 15 / 85$ & 231 & 15.20 & -0.58 & -0.60 & -0.56 & -0.76 & -0.63 \\
\hline $4 / 19 / 85$ & 235 & 15.33 & -0.62 & -0.65 & -0.59 & -0.72 & -0.64 \\
\hline $4 / 22 / 85$ & 238 & 15.43 & -0.57 & -0.59 & -0.54 & -0.72 & -0.61 \\
\hline $4 / 26 / 85$ & 242 & 15.56 & -0.61 & -0.63 & -0.55 & -0.74 & -0.63 \\
\hline $4 / 29 / 85$ & 245 & 15.65 & -0.57 & -0.60 & -0.56 & -0.74 & -0.62 \\
\hline $5 / 03 / 85$ & 249 & 15.78 & -0.56 & -0.58 & -0.54 & -0.72 & -0.60 \\
\hline $5 / 06 / 85$ & 252 & 15.87 & -0.57 & -0.60 & -0.60 & -0.78 & -0.64 \\
\hline $5 / 10 / 85$ & 256 & 16.00 & -0.57 & -0.58 & -0.55 & -0.74 & -0.61 \\
\hline $5 / 13 / 85$ & 259 & 16.09 & -0.58 & -0.59 & -0.56 & -0.71 & -0.61 \\
\hline $5 / 17 / 85$ & 263 & 16.22 & -0.56 & -0.60 & -0.57 & -0.73 & -0.62 \\
\hline $5 / 20 / 85$ & 266 & 16.31 & -0.55 & -0.56 & -0.53 & -0.71 & -0.59 \\
\hline
\end{tabular}


TABLE XV (cont'd) MOISTURE DESORPTION OF TAIL ROTOR SPAR S/N A-116-00178, BUTTLINES 5-7, B END

\begin{tabular}{|c|c|c|c|c|c|c|c|}
\hline $\begin{array}{l}\text { DATE OF } \\
\text { WEIGHING }\end{array}$ & DAYS & $\begin{array}{l}\text { SQ RT } \\
\text { OF TIME }\end{array}$ & $\begin{array}{l}\% \text { MOIST } \\
\text { DESORBED } \\
\text { COUPON } \\
5-7-1\end{array}$ & $\begin{array}{l}\% \text { MOIST } \\
\text { DESORBED } \\
\text { COUPON } \\
5-7-2\end{array}$ & $\begin{array}{l}\% \text { MOIST } \\
\text { DESORBED } \\
\text { COUPON } \\
5-7-3\end{array}$ & $\begin{array}{l}\% \text { MOIST } \\
\text { DESORBED } \\
\text { COUPON } \\
5-7-4\end{array}$ & $\begin{array}{l}\text { AVERAGE } \\
\% \text { MOIST } \\
\text { DESORBED }\end{array}$ \\
\hline $5 / 24 / 85$ & 270 & 16.43 & -0.59 & -0.64 & -0.58 & -0.78 & -0.65 \\
\hline $5 / 31 / 85$ & 277 & 16.64 & -0.58 & -0.61 & -0.57 & -0.74 & -0.62 \\
\hline $6 / 03 / 85$ & 280 & 16.73 & -0.60 & -0.60 & -0.55 & -0.75 & -0.62 \\
\hline $6 / 07 / 85$ & 284 & 16.85 & -0.60 & -0.64 & -0.59 & -0.78 & -0.65 \\
\hline $6 / 10 / 85$ & 287 & 16.94 & -0.56 & -0.58 & -0.56 & -0.73 & -0.61 \\
\hline $6 / 14 / 85$ & 291 & 17.06 & -0.66 & -0.69 & -0.65 & -0.85 & -0.71 \\
\hline $6 / 17 / 85$ & 297 & 17.23 & -0.56 & -0.58 & -0.52 & -0.70 & -0.59 \\
\hline $6 / 21 / 85$ & 301 & 17.35 & -0.58 & -0.60 & -0.53 & -0.74 & -0.61 \\
\hline $6 / 24 / 85$ & 304 & 17.44 & -0.58 & -0.59 & -0.54 & -0.74 & -0.61 \\
\hline $6 / 28 / 85$ & 308 & 17.55 & -0.56 & -0.57 & -0.54 & -0.72 & -0.60 \\
\hline $7 / 05 / 85$ & 315 & 17.75 & -0.55 & -0.56 & -0.52 & -0.70 & -0.58 \\
\hline $7 / 08 / 85$ & 318 & 17.83 & -0.57 & -0.59 & -0.55 & -0.73 & -0.61 \\
\hline $7 / 12 / 85$ & 322 & 17.94 & -0.60 & -0.60 & -0.58 & -0.74 & -0.63 \\
\hline $7 / 15 / 85$ & 325 & 18.03 & -0.66 & -0.59 & -0.54 & -0.70 & -0.62 \\
\hline $7 / 19 / 85$ & 329 & 18.14 & -0.59 & -0.60 & -0.56 & -0.74 & -0.62 \\
\hline $7 / 22 / 85$ & 332 & 18.22 & -0.57 & -0.58 & -0.54 & -0.73 & -0.61 \\
\hline $7 / 26 / 85$ & 336 & 18.33 & -0.57 & -0.61 & -0.54 & -0.71 & -0.61 \\
\hline $8 / 02 / 85$ & 343 & 18.52 & -0.59 & -0.61 & -0.58 & -0.76 & -0.63 \\
\hline $8 / 05 / 85$ & 346 & 18.60 & -0.59 & -0.61 & -0.58 & -0.76 & -0.64 \\
\hline $8 / 09 / 85$ & 350 & 18.71 & -0.58 & -0.59 & -0.54 & -0.72 & -0.61 \\
\hline $8 / 12 / 85$ & 353 & 18.79 & -0.56 & -0.58 & -0.55 & $-0 / 71$ & -0.60 \\
\hline $8 / 16 / 85$ & 357 & 18.89 & -0.59 & -0.60 & -0.55 & -0.74 & -0.62 \\
\hline $8 / 19 / 85$ & 360 & 18.97 & -0.59 & -0160 & -0.57 & -0.77 & -0.63 \\
\hline $8 / 23 / 85$ & 364 & 19.08 & -0.58 & -0.61 & -0.56 & -0.74 & -0.62 \\
\hline $8 / 26 / 85$ & 367 & 19.16 & -0.55 & -0.58 & -0.53 & -0.70 & -0.59 \\
\hline $8 / 30 / 85$ & 371 & 19.26 & -0.56 & -0.57 & -0.53 & -0.73 & -0.60 \\
\hline $9 / 06 / 85$ & 378 & 19.44 & -0.54 & -0.57 & -0.53 & -0.72 & -0.59 \\
\hline $9 / 09 / 85$ & 382 & 19.54 & -0.56 & -0.55 & -0.51 & -0.68 & -0.57 \\
\hline $9 / 13 / 85$ & 386 & 19.65 & -0.58 & -0.61 & -0.56 & -0.76 & -0.63 \\
\hline $9 / 16 / 85$ & 389 & 19.72 & -0.58 & -0.58 & -0.54 & -0.75 & -0.61 \\
\hline $9 / 20 / 85$ & 393 & 19.82 & -0.57 & -0.57 & -0.52 & -0.71 & -0.59 \\
\hline $9 / 23 / 85$ & 396 & 19.90 & -0.59 & -0.61 & -0.54 & -0.76 & -0.63 \\
\hline $9 / 27 / 85$ & 400 & 20.00 & -0.58 & -0.60 & -0.54 & -0.73 & -0.61 \\
\hline $9 / 30 / 85$ & 403 & 20.07 & -0.55 & -0.58 & -0.53 & -0.70 & -0.59 \\
\hline $10 / 04 / 85$ & 407 & 20.17 & -0.59 & -0.63 & -0.58 & -0.75 & -0.64 \\
\hline $10 / 07 / 85$ & 410 & 20.25 & -0.55 & -0.58 & -0.54 & -0.75 & -0.60 \\
\hline $10 / 14 / 85$ & 417 & 20.42 & -0.54 & -0.57 & -0.51 & -0.69 & -0.58 \\
\hline
\end{tabular}


TABLE XV (cont'd) MOISTURE DESORPTION OF TAIL ROTOR SPAR S/N A-116-00178, BUTTLINES 5-7, B END

\begin{tabular}{lccccccc}
\hline & & & $\begin{array}{c}\% \text { MOIST } \\
\text { DESORBED } \\
\text { COUPON }\end{array}$ & $\begin{array}{l}\% \text { MOIST } \\
\text { DESORBED } \\
\text { COUPON }\end{array}$ & $\begin{array}{l}\text { \% MOIST } \\
\text { DESORBED } \\
\text { COUPON }\end{array}$ & $\begin{array}{l}\% \text { MOIST } \\
\text { DESORBED } \\
\text { COUPON }\end{array}$ & $\begin{array}{l}\text { AVERAGE } \\
\% \text { MOIST }\end{array}$ \\
WEIGHING & DAYS & SQ R TIME & $5-7-1$ & $5-7-2$ & $5-7-3$ & $5-7-4$ & DESORBED \\
\hline $10 / 18 / 85$ & 421 & 20.52 & -0.58 & -0.63 & -0.57 & -0.74 & -0.63 \\
$10 / 21 / 85$ & 424 & 20.59 & -0.55 & -0.57 & -0.52 & -0.71 & -0.59 \\
$10 / 25 / 85$ & 428 & 20.69 & -0.61 & -0.64 & -0.59 & -0.75 & -0.65 \\
$11 / 01 / 85$ & 435 & 20.86 & -0.57 & -0.56 & -0.52 & -0.71 & -0.59 \\
$11 / 04 / 85$ & 438 & 20.93 & -0.56 & -0.58 & -0.54 & -0.71 & -0.60 \\
$11 / 08 / 85$ & 442 & 21.02 & -0.58 & -0.58 & -0.56 & -0.77 & -0.62 \\
$11 / 11 / 85$ & 445 & 21.10 & -0.59 & -0.57 & -0.55 & -0.71 & -0.61 \\
$11 / 15 / 85$ & 449 & 21.19 & -0.57 & -0.59 & -0.53 & -0.71 & -0.60 \\
$11 / 18 / 85$ & 452 & 21.26 & -0.57 & -0.56 & -0.52 & -0.71 & -0.59 \\
$11 / 22 / 85$ & 456 & 21.35 & -0.57 & -0.60 & -0.55 & -0.74 & -0.62 \\
$12 / 02 / 85$ & 466 & 21.59 & -0.58 & -0.59 & -0.52 & -0.74 & -0.61 \\
$12 / 06 / 85$ & 470 & 21.68 & -0.57 & -0.60 & -0.55 & -0.73 & -0.61 \\
$12 / 09 / 85$ & 473 & 21.75 & -0.59 & -0.60 & -0.56 & -0.74 & -0.62 \\
$12 / 13 / 85$ & 477 & 21.84 & -0.58 & -0.59 & -0.59 & -0.76 & -0.63 \\
$12 / 16 / 85$ & 480 & 21.91 & -0.57 & -0.59 & -0.54 & -0.74 & -0.61 \\
$12 / 20 / 85$ & 484 & 22.00 & -0.57 & -0.61 & -0.52 & -0.72 & -0.61
\end{tabular}




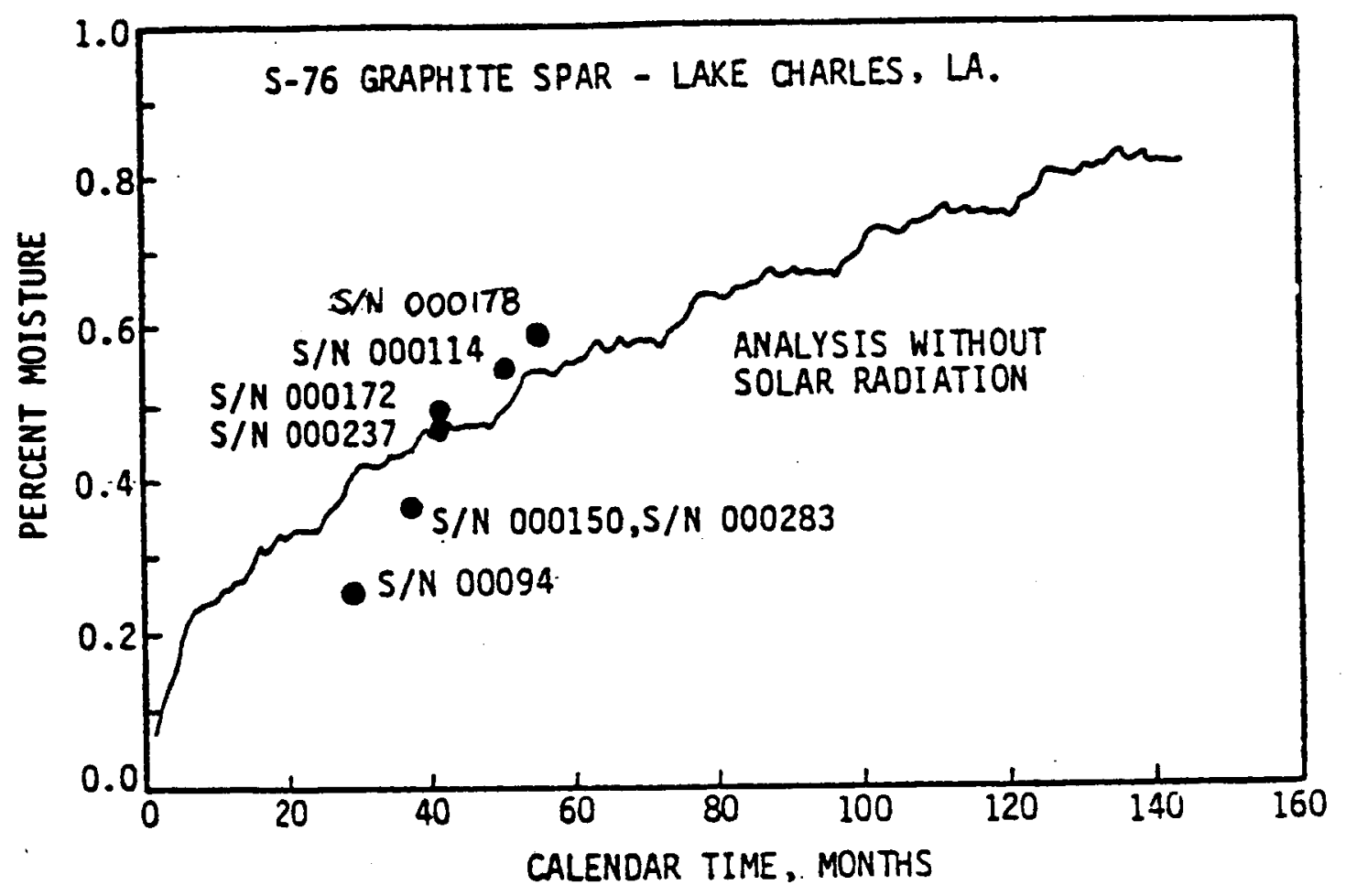

FIGURE 11. MEASURED MOISTURE VERSUS ANALYSIS FOR GRAPHITE/EPOXY TAIL ROTOR SPARS 


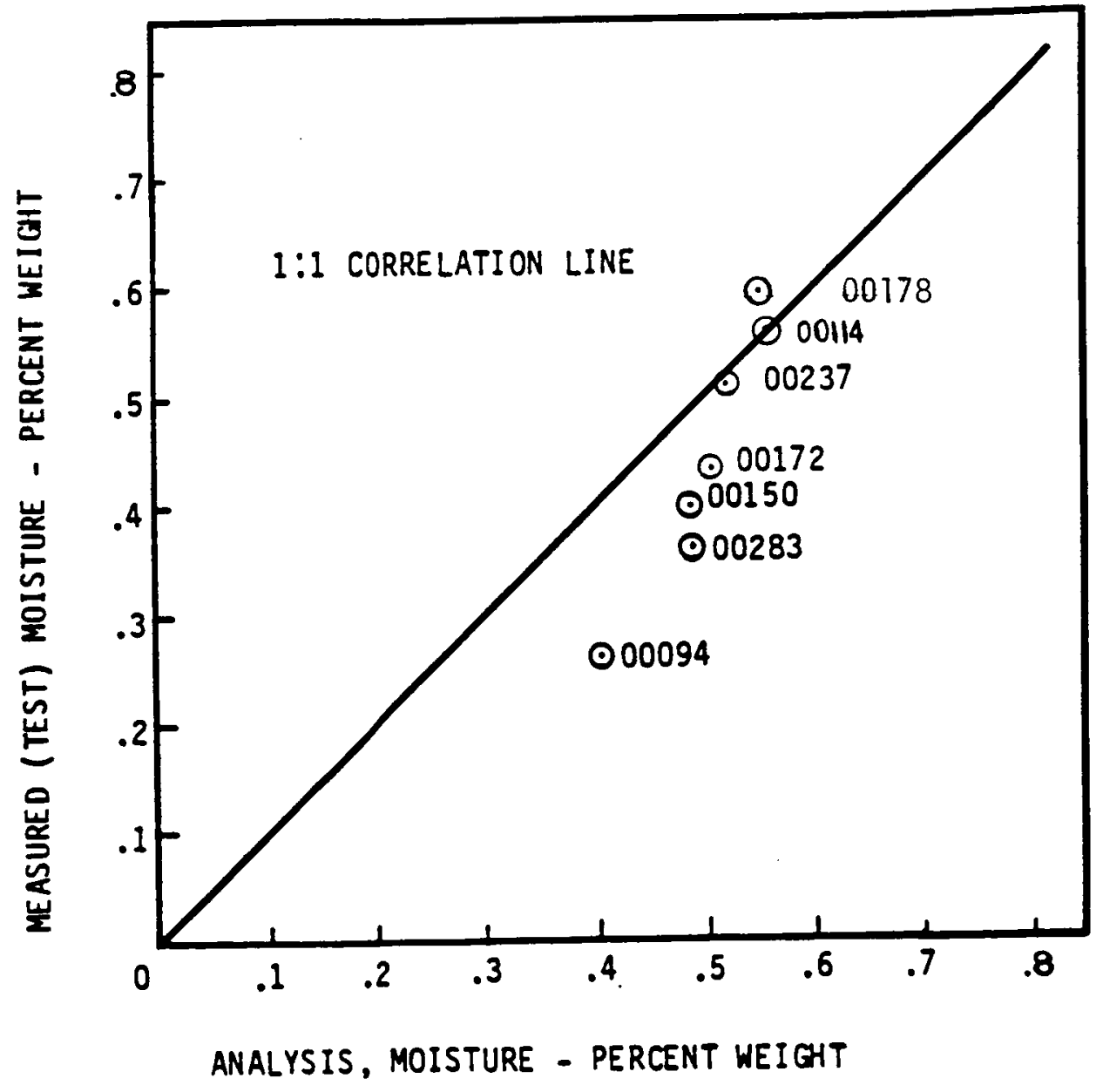

FIGURE 12. CORRELATION BETWEEN MEASURED MOISTURE AND ANALYSIS FOR GRAPHITE/EPOXY TAIL ROTOR SPARS 
4.1 Field Exposed Panels

As part of a Sikorsky internal research and development program, entitled the Life Extension Program for Composite Structures, AS1/ 6350 graphite/epoxy and $285 / 5143 \mathrm{Kevlar} /$ epoxy panels are exposed to the environment in two weathering locations: West Palm Beach, Florida and Stratford, Connecticut. Three graphite/epoxy panel configurations are exposed as part of this evaluation: 6, 14 and 33 ply panels, with a nominal per ply thickness of 0.012 inch. One Kevlar/epoxy configuration is examined: 5 ply panels, having a nominal per ply thickness of 0.009 inch. The ply configurations of the panels are representative of S-76 components. Data is presented herein for comparison purposes.

\subsubsection{Moisture Measurements}

Panels are returned from the weathering locations annually to determine moisture content and mechanical properties. During this reporting period, panels having four and five years exposure to the environment were returned for evaluation.

Moisture coupons, removed from exposed panels, are desorbed in an environmentally controlled chamber at $150 \pm 2^{\circ} \mathrm{F}$. A summary of the moisture measurements for panels with two, three, four and five years of exposure is presented in Table XVI. Inspection of the table shows 6 ply panels have reached saturation at levels in agreement with the 1.1 percent design moisture level for AS-1/6350 graphite/epoxy. Fourteen and thirty-three ply panels are not expected to reach saturation for several years.

A comparison of predicted versus measured moisture content for six ply panels weathered in Stratford, Connecticut is typical, and presented in Figure 13. As seen in the plot, measured moisture values are in good accord with predicted levels.

4.2 Coupon Strength Tests

\subsubsection{Static Strength}

Coupons were also removed from the environmentally exposed panels for mechanical testing. Flexural shear, static short beam shear, and short beam shear fatigue tests were conducted on graphite/epoxy specimens. The static flexural properties were determined in accordance with ASTM D 790, Reference (5). Static and fatigue short beam shear strengths were determined in accordance with the methods previously described. Tensile tests were conducted on Kevlar/epoxy coupons, in accordance with ASTM D 3039, Reference (6). Specimen configurations were as shown in Figure 14. Results of all coupon tests are summarized in Table XVII. 


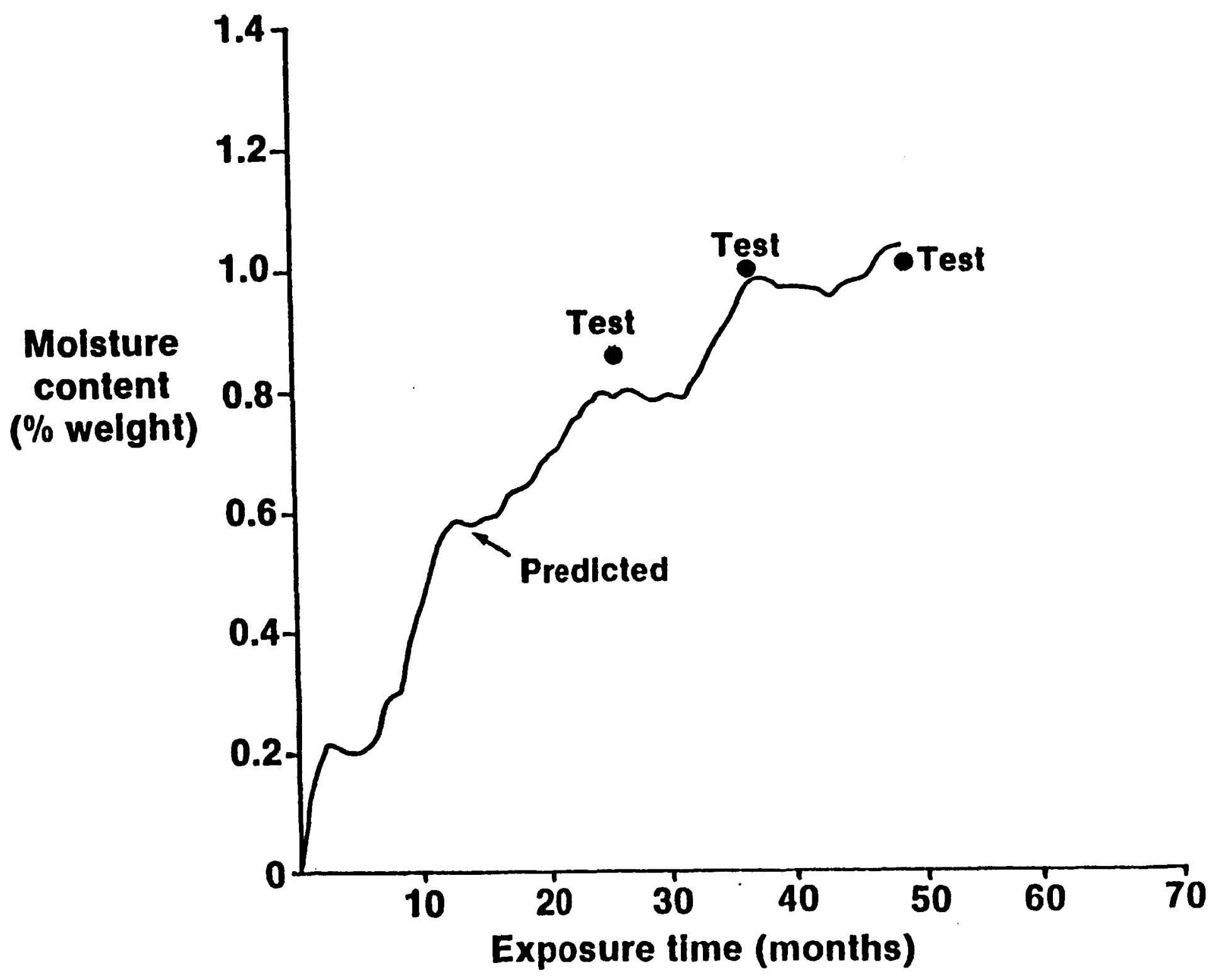

FIGURE 13. MEASURED AND PREDICTED MOISTURE LEVEL FOR SIX PLY AS 1/6350 GRAPHITE EPOXY PANELS (WEATHERED IN STRATFORD, CONNECTICUT) 
TABLE XVI SUMMARY OF MOISTURE MEASUREMENTS FOR FIELD EXPOSED PANELS

\begin{tabular}{|c|c|c|c|c|}
\hline MATERIAL & $\begin{array}{l}\text { NUMBER } \\
\text { OF } \\
\text { PLIES }\end{array}$ & $\begin{array}{l}\text { EXPOSURE } \\
\text { LOCATION }\end{array}$ & $\begin{array}{l}\text { EXPOSURE } \\
\text { TIME } \\
\text { (MONTHS) }\end{array}$ & $\begin{array}{l}\text { PERCENT } \\
\text { MOISTURE } \\
\text { (BY WEIGHT) }\end{array}$ \\
\hline $\begin{array}{l}\text { AS1/6350 } \\
\text { GRAPHITE/ } \\
\text { EPOXY }\end{array}$ & 6 & WPB & $\begin{array}{l}26 \\
35 \\
48.5 \\
60.5\end{array}$ & $\begin{array}{l}1.02 \\
1.23 \\
1.15 \\
\text { DIP }\end{array}$ \\
\hline & 6 & STRATFORD & $\begin{array}{l}25 \\
36 \\
49 \\
62\end{array}$ & $\begin{array}{l}0.86 \\
1.00 \\
0.99 \\
\text { DIP: }\end{array}$ \\
\hline $\begin{array}{l}\text { AS 1/6350 } \\
\text { GRAPHITE/ } \\
\text { EPOXY }\end{array}$ & 14 & $\begin{array}{l}\text { STRATFORD } \\
\text { STRATFORD }\end{array}$ & $\begin{array}{l}25 \\
34.5 \\
48 \\
61\end{array}$ & $\begin{array}{l}0.37 \\
0.48 \\
0.44 \\
\text { DIP* }\end{array}$ \\
\hline $\begin{array}{l}\text { AS1/6350 } \\
\text { GRAPHITE/ } \\
\text { EPOXY }\end{array}$ & 33 & $\begin{array}{l}\text { WPB } \\
\text { WPB } \\
\text { STRATFORD } \\
\text { STRATFORD }\end{array}$ & $\begin{array}{l}26 \\
35 \\
48.5 \\
60.5 \\
\\
25 \\
36 \\
49.5 \\
62\end{array}$ & $\begin{array}{l}0.27 \\
0.37 \\
0.35 \\
\text { DIP* } \\
0.18 \\
0.22 \\
0.24 \\
\text { DIP* }\end{array}$ \\
\hline $\begin{array}{l}285 / 5143 \\
\text { KEVLAR/ } \\
\text { EPOXY }\end{array}$ & 5 & $\begin{array}{l}\text { WPB } \\
\text { WPB }\end{array}$ & $\begin{array}{l}26 \\
35 \\
48.5 \\
60.5\end{array}$ & $\begin{array}{l}1.56 \\
2.08 \\
1.90 \\
\text { DIP* }\end{array}$ \\
\hline & 5 & STRATFORD & $\begin{array}{l}26 \\
37 \\
50 \\
63\end{array}$ & $\begin{array}{l}1.53 \\
1.72 \\
1.75 \\
\text { DIP* }\end{array}$ \\
\hline
\end{tabular}

NOTE: DIP* - DESORPTION IN PROGRESS 


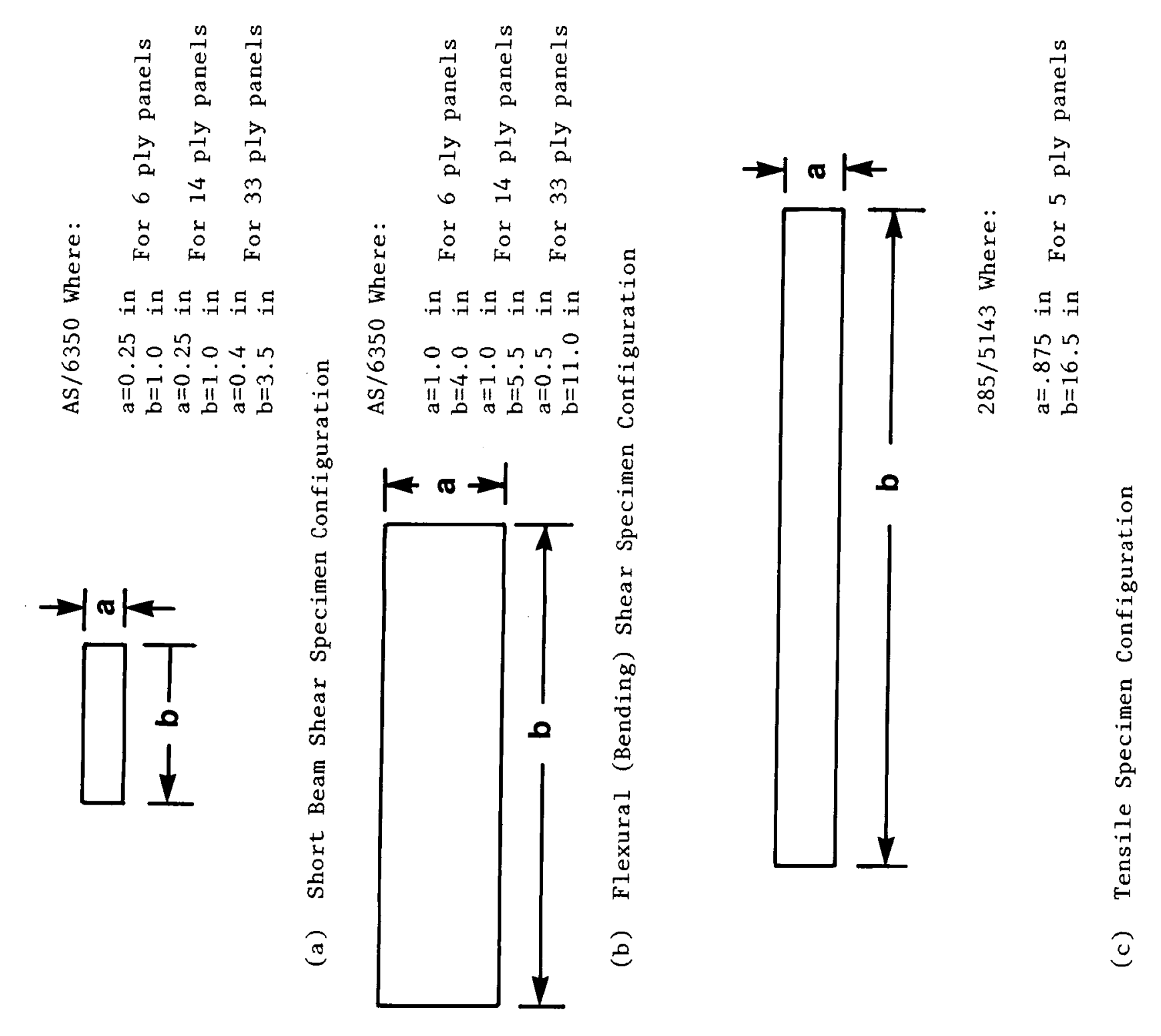

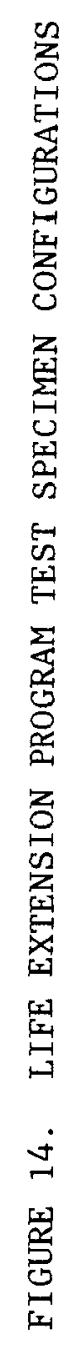




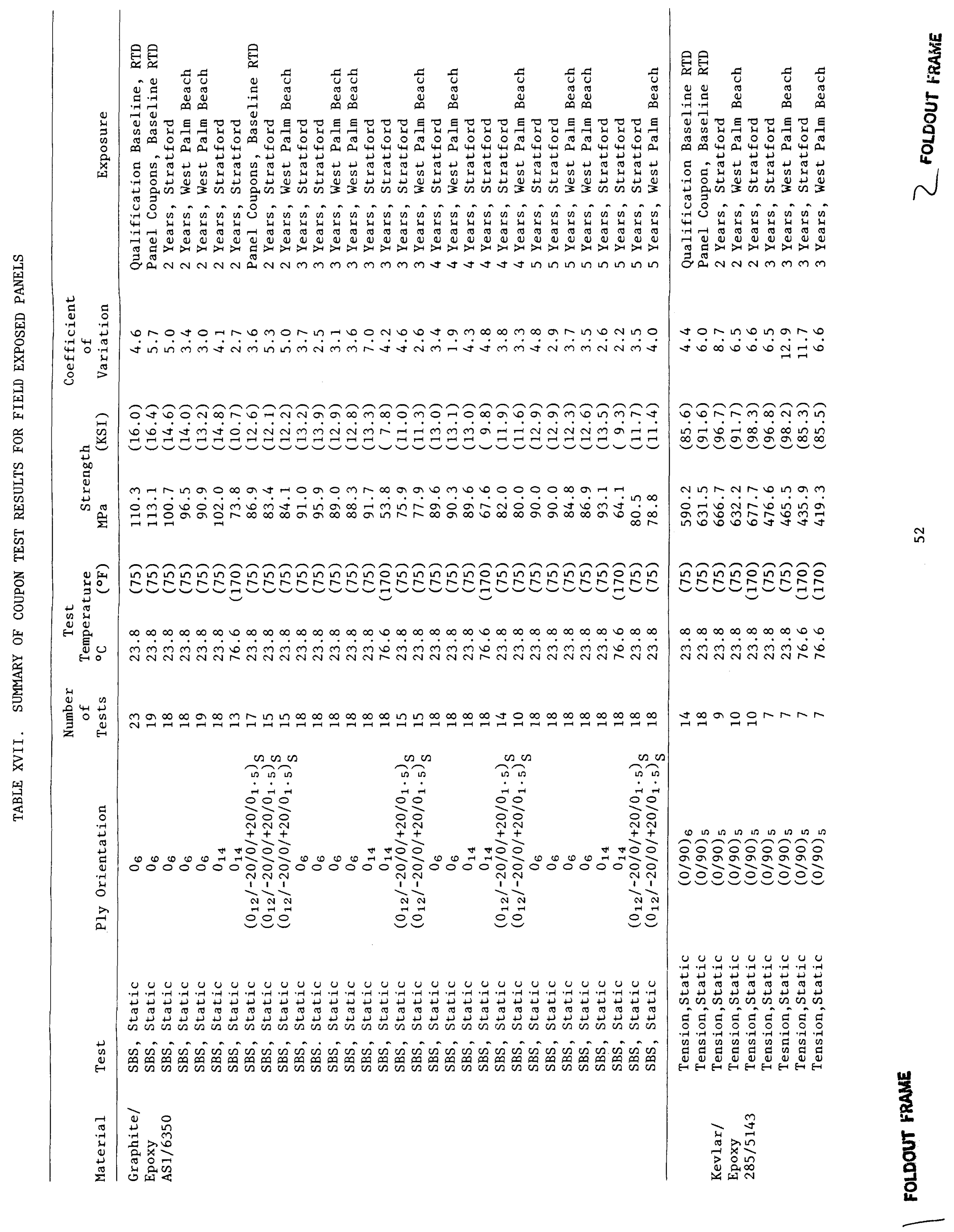




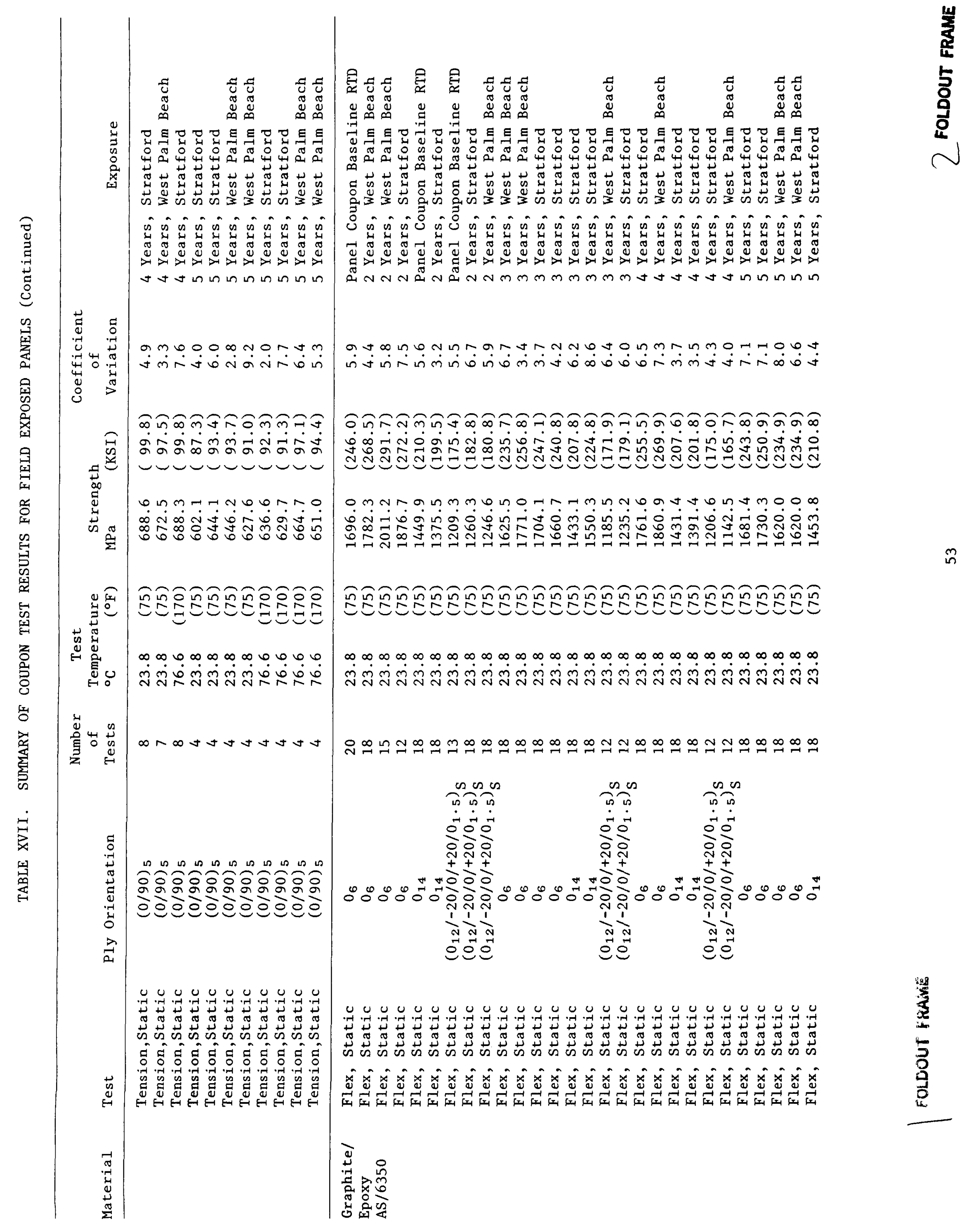



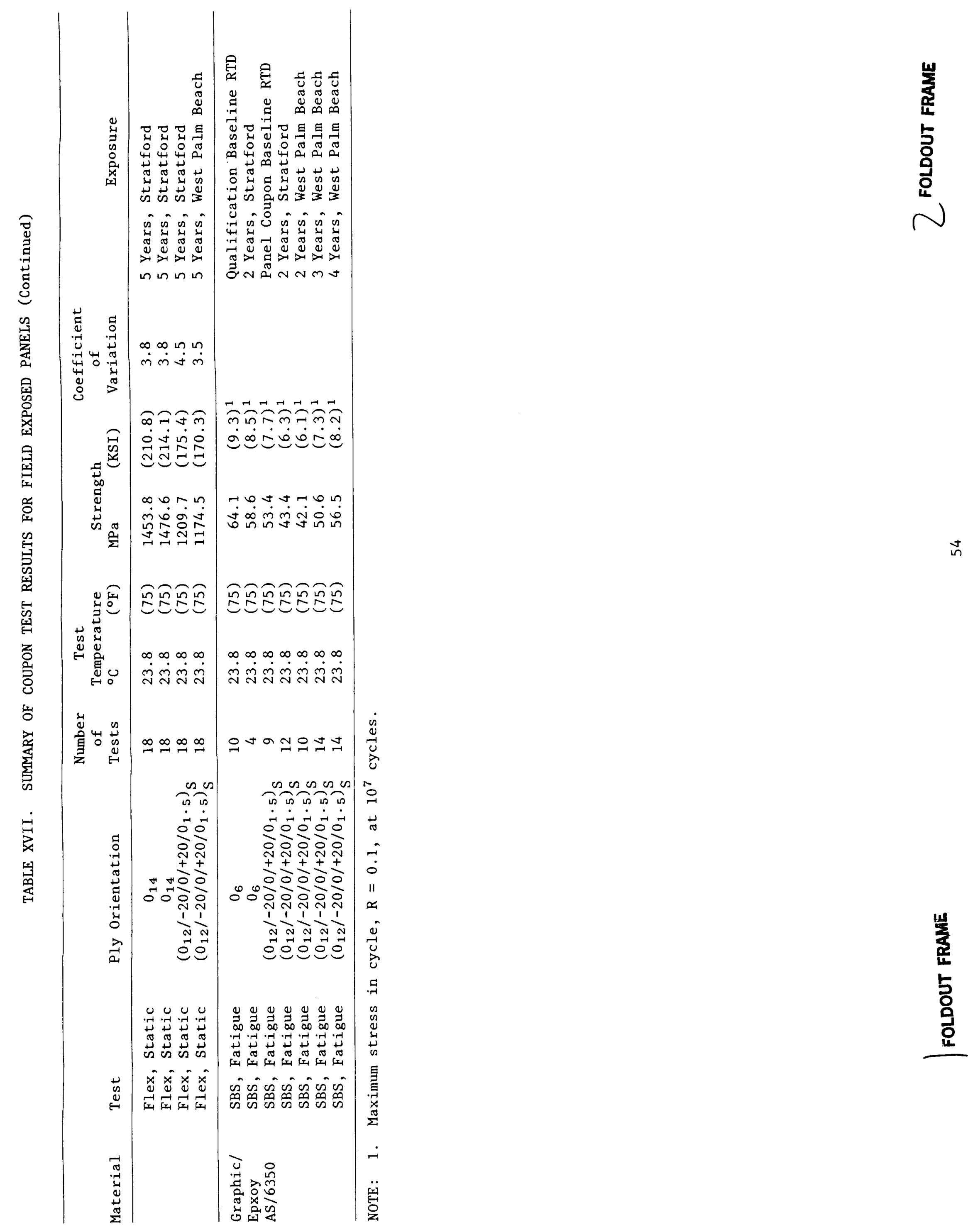
5.0 ANALYSIS OF TEST RESULTS

$5.1 \quad$ Environmental Factor Trends

In evaluating environmental effects on composite materials, results of component coupon and panel testing are compared to environmental factor trends determined previously for AS1/6350 graphite/epoxy and $285 / 5143 \mathrm{Kevlar} /$ epoxy. The static environmental factor is defined as the ratio of mean strength at the environmental condition to the mean room temperature dry strength.

Figure 15 compares AS1/6350 panel test results with a plot of the environmental factor trends for interlaminar shear. Test data falls in accord with laboratory predictions. Figures 16 and 17 compare panel test results with plots of the environmental factor trends for flexural shear and tensile strengths, for AS1/6350 and 285/5143 respectively. Examination of the plots shows results obtained in panel tests to be consistently above environmental factor requirements. 


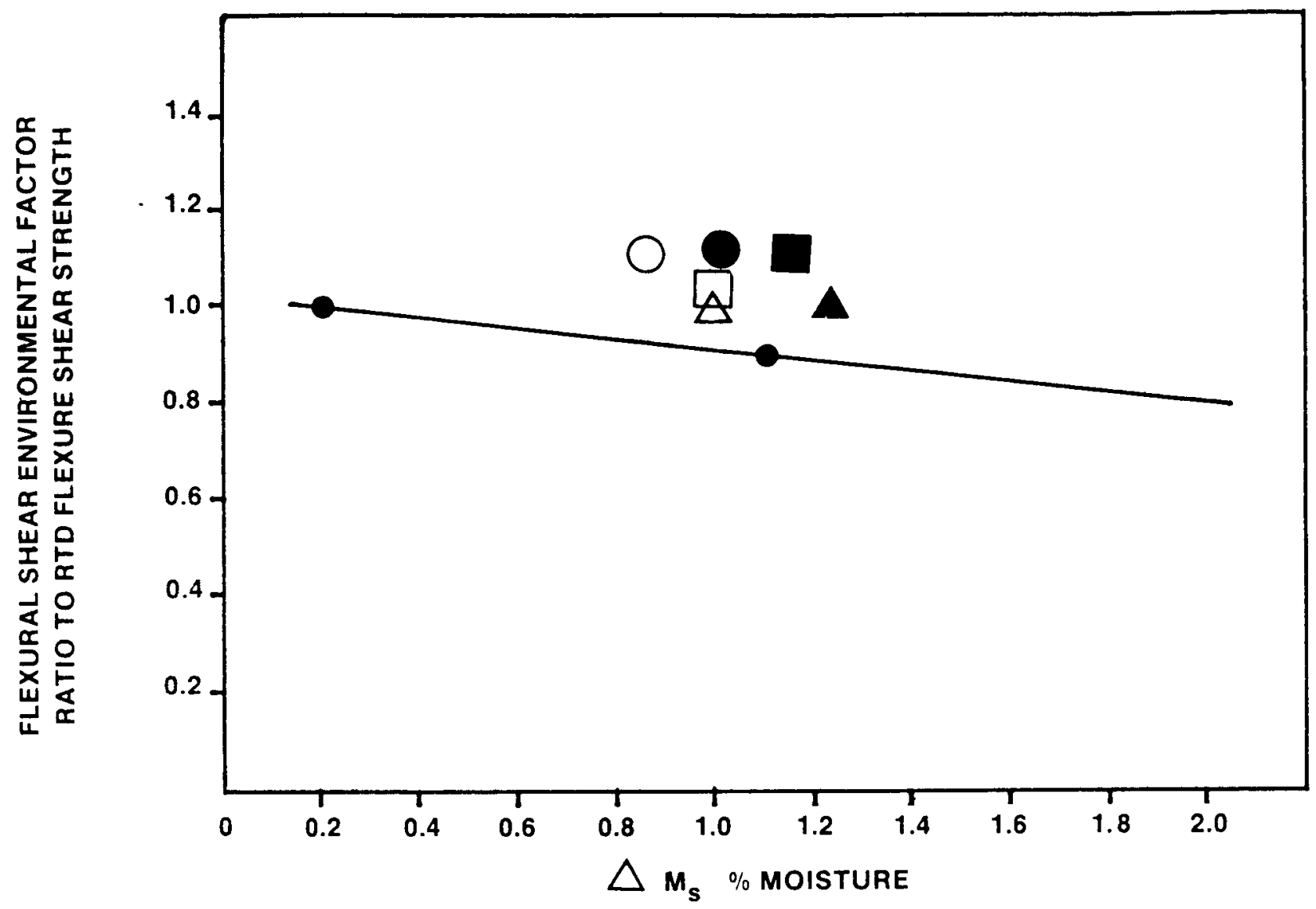

- ENVIRONMENTAL FActor test DATA 6 PLy PANEL

2 YEAR PANEL DATA, WEATHERED IN

STRATFORD

3 YEAR PANEL DATA, WEATHERED IN

$\triangle$ STRATFORD

4 YEAR PANEL DATA, WEATHERED IN STRATFORD

WEST PALM BEACH

A West PALM BEACH WEST PALM BEACH

FIGURE 15 COMPARISON OF LIFE EXTENSION PROGRAM FLEXURAL SHEAR ENVIRONMENTAL FACTOR DATA WITH AS1/6350 ENVIRONMENTAL FACTOR TRENDS 


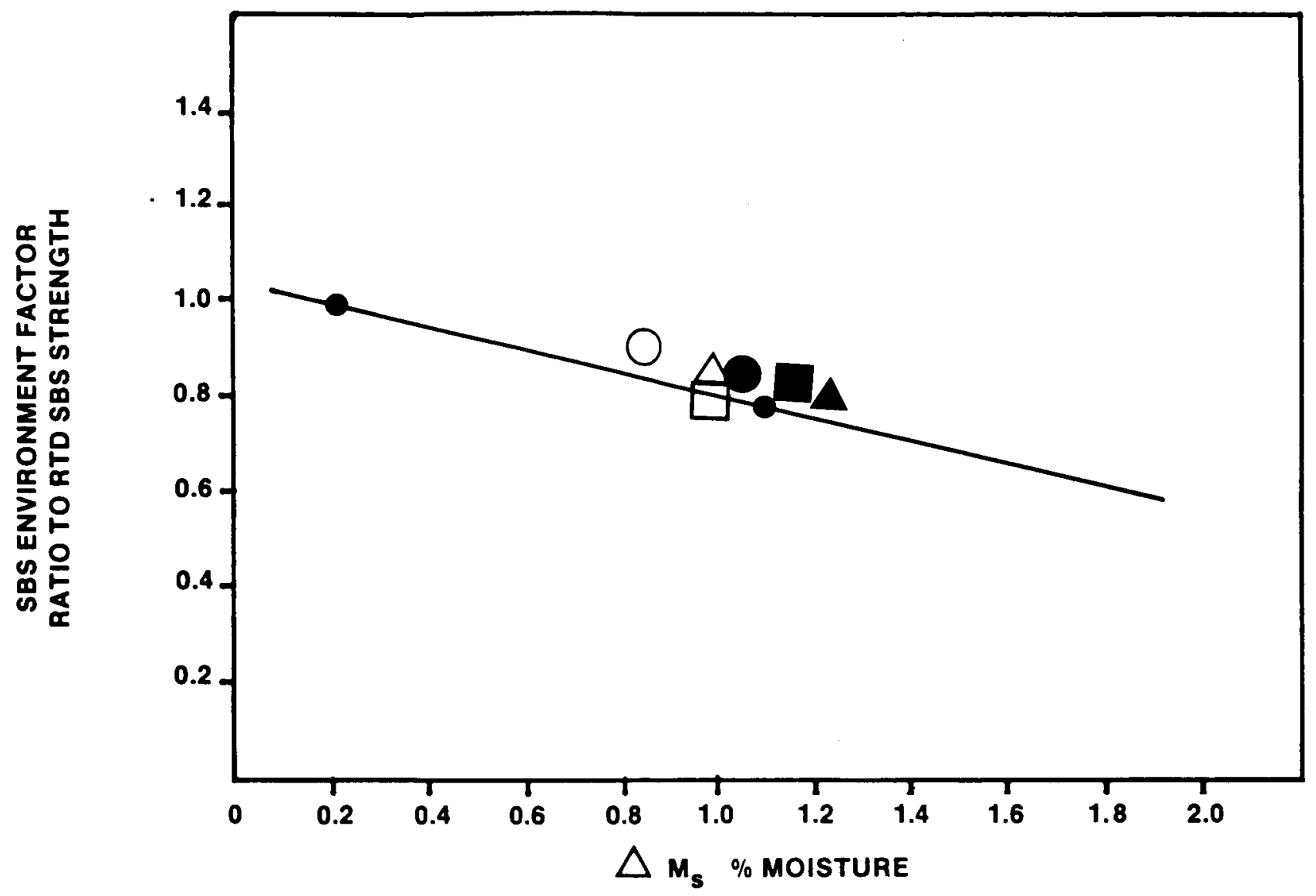

- ENVIRONMENTAL FACTOR TEST DATA 6 PLY PANEL

2 YEAR PANEL DATA, WEATHERED IN

STRATFORD

3 YEAR PANEL DATA, WEATHERED IN $\triangle$ STRATFORD WEST PALM BEACH 4 YEAR PANEL DATA, WEATHERED IN STRATFORD WEST PALM BEACH WEST PALM BEACH

FIGURE 16 COMPARISON OF LIFE EXTENSION PROGRAM INTERFAMINAR SHEAR (STATIC) ENVIRONMENTAL FACTOR DATA WITH AS1/6350 ENVIRONMENTAL FACTOR TRENDS 


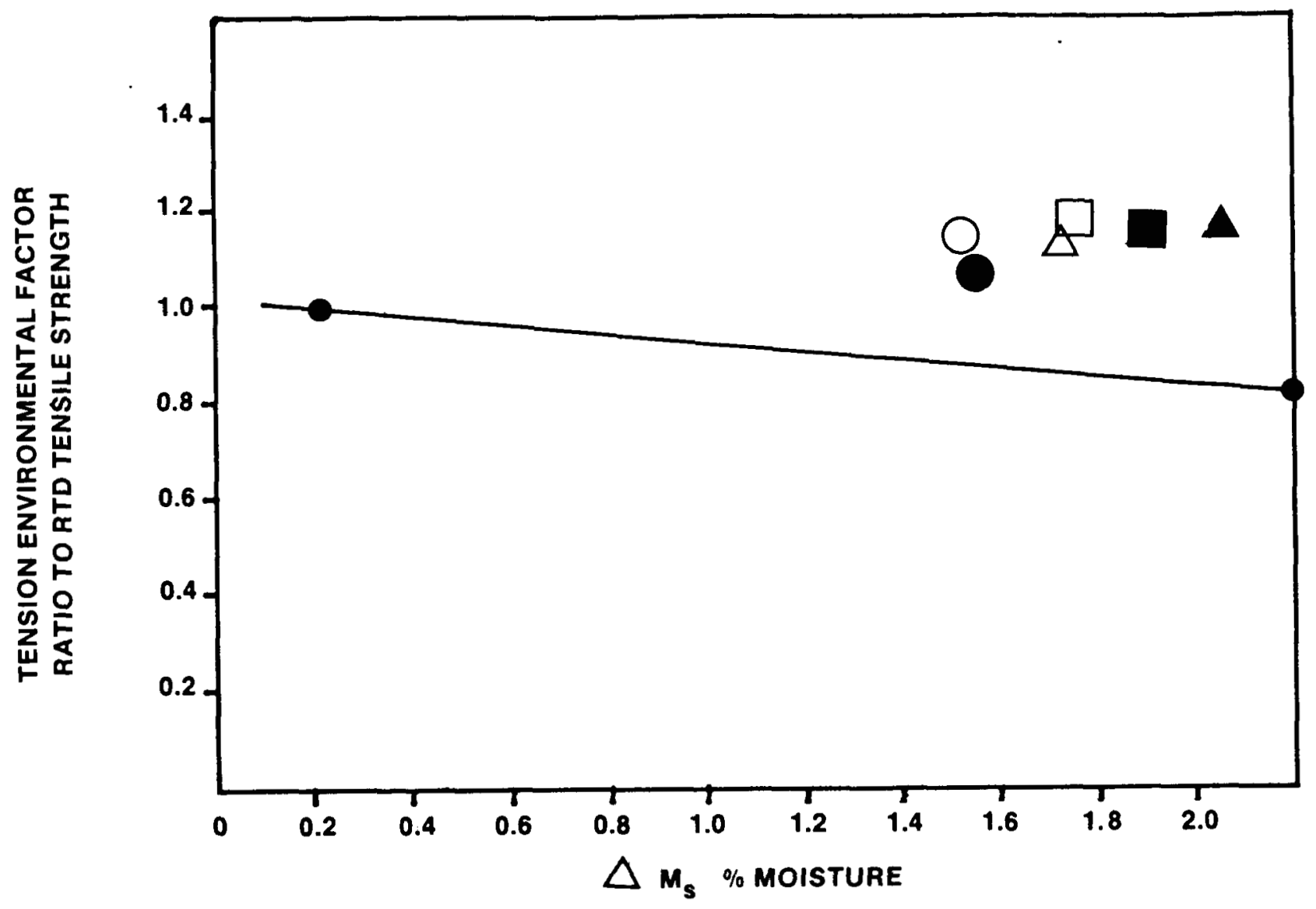

- environmental factor test data 5 PLy panel

2 YEAR PANEL DATA, WEATHERED IN

STRATFORD

WEST PALM BEACH

3 YEAR PANEL DATA, WEATHERED IN

$\triangle$ STRATFORD

WEST PALM BEACH

4 YEAR PANEL DATA, WEATHERED IN

STRATFORD

WEST PALM BEACH

FIGURE 17 COMPARISON OF LIFE EXTENSION PROGRAM TENSION

ENVIRONMENTAL FACTOR DATA WTTH $285 / 5143$

ENVIRONMENTAL FACTOR TRENDS 


\section{$7.0 \quad$ REFERENCES}

$\underline{\text { References }}$

(1)

\section{Title}

Rich, M. and Lowry, D. "Flight Service Evaluation of Composite Helicopter Components," First Annual Report March 1981 through April 1982, NASA CR-165952, (SER-510089) June 1982

Rich, M. and Lowry, D. "Flight Service Evaluation of Composite Helicopter Components" Second Annual Report May 1982 through September 1983, NASA CR-172562 (SER-510117), April 1985

ASTM D 2344-84, "Standard Test Method for Apparent Horizontal Shear Strength of Reinforced Plastics by Short Beam Method"

"Modern Elementary Statistics" John E. Freund, 1979 PrenticeHall, Incorporated, Englewood Cliffs, New Jersey 07632, 1977

ASTM D 790-81, "Standard Methods of Test for Flexural Properties of Plastics"

ASTM D 3039-84, "Standard Test Method for Tensile Properties of Oriented Fiber Composites" 


\section{$6.0 \quad$ CONCLUSIONS}

$6.1 \quad$ Conclusions

The following conclusions are based on the results of composite components and panels having up to five years of environmental exposure that have been returned from the field for evaluation during this reporting period.

1. Full scale testing of two stabilizers, $\mathrm{S} / \mathrm{N}$ B-157-00009 and $\mathrm{S} / \mathrm{N}$ B-157-00021 produced fractures in the stabilizer torque box between Buttline 3 Left and Buttline 3 Right as expected.

2. Coupons removed from stabilizer S/N B-157-00009 for desorption indicated moisture levels comparable to 14 ply graphite/epoxy panels having 48 months exposure to the environment in Stratford, Connecticut. Desorption of moisture coupons removed from stabilizer $\mathrm{S} / \mathrm{N} \mathrm{B-157-00021} \mathrm{is} \mathrm{in} \mathrm{progress.}$

3. Small scale coupon tests of tail rotor spar S/N A-116-00178 generated results that were somewhat (8-14 percent) higher than expected when compared with past spar coupon testing, but consistent with panel coupon data having 48 month environmental exposure.

4. Results of panel coupon testing supported laboratory predictions for environmental factor trends. 
Standard Bibliographic Page

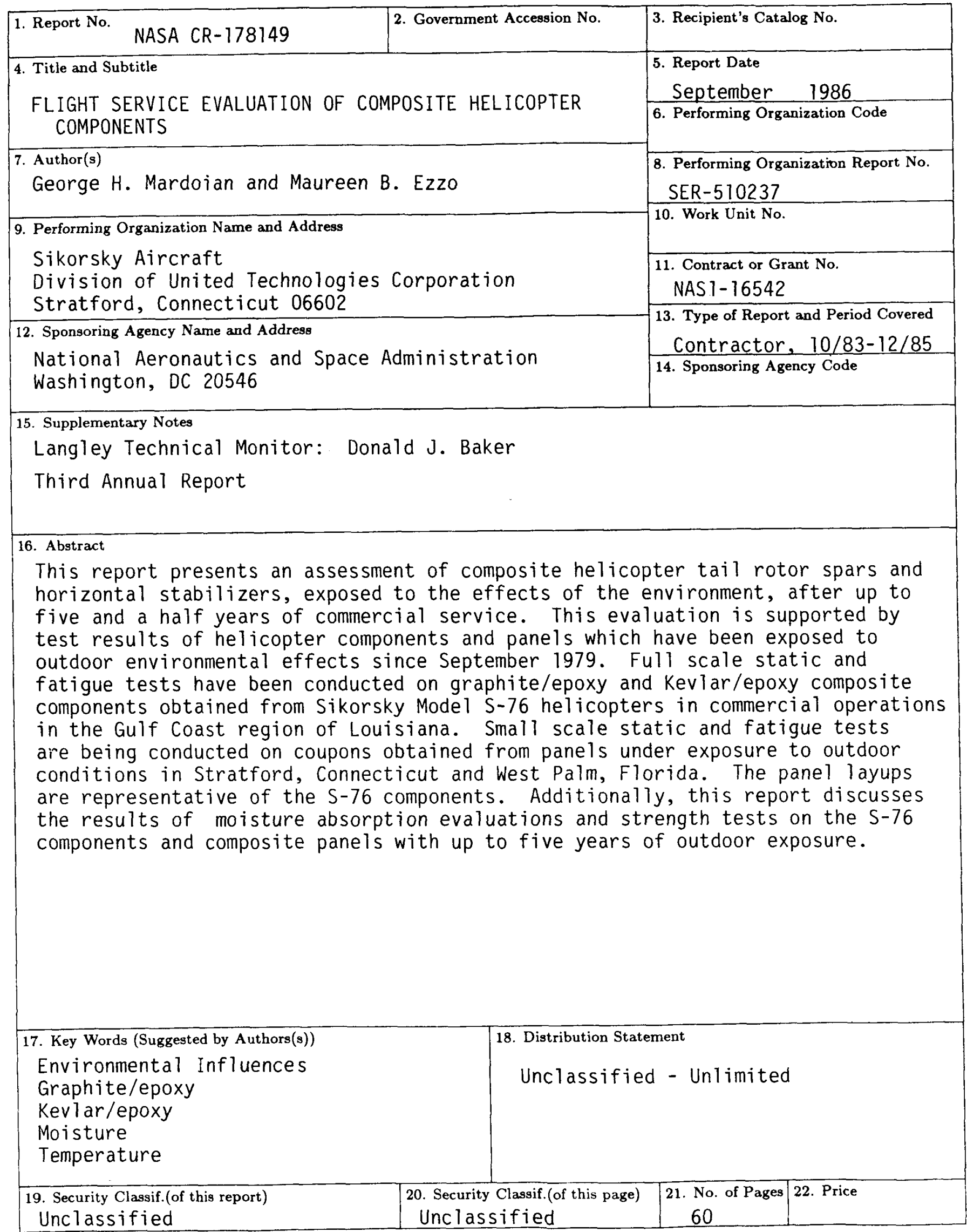

